Elementos da teoria de Teichmüller

\author{
Eber Daniel Chuño Vizarreta
}



SERVIÇO DE PÓS-GRADUAÇÃO DO ICMC-USP

Data de Depósito:

Assinatura:

\title{
Elementos da teoria de Teichmüller
}

\author{
Eber Daniel Chuño Vizarreta
}

Orientador: Prof. Dr. Igor Mencattini

Dissertação apresentada ao Instituto de Ciências Matemáticas e de Computação - ICMC-USP, como parte dos requisitos para obtenção do título de Mestre em Ciências - Matemática . EXEMPLAR DE DEFESA 
Ficha catalográfica elaborada pela Biblioteca Prof. Achille Bassi e Seção Técnica de Informática, ICMC/USP, com os dados fornecidos pelo(a) autor(a)

Chuño Vizarreta, Eber Daniel
Elementos da teoria de Teichmüller / Eber Daniel
Chuño Vizarreta; orientador Igor Mencattini -- São
Carlos, 2011.
$86 \mathrm{p.}$
Dissertação (Mestrado - Programa de Pós-Graduação en
Matemática) -- Instituto de Ciências Matemáticas e
de Computação, Universidade de São Paulo, 2011.
1. Epaço de Teichmüller. 2. Coordenadas de Fenchel-
Nielsen. 3. Espaço de Fricke. 4. Superfície de
Riemann. 5. Grupo Fuchsiano. I. Mencattini, Igor,
orient. II. Título.




\section{Agradecimentos}

Gostaria de expressar aqui minha gratidão em primeiro lugar a Deus, pelo grande dom da Vida, que me permitiram chegar até onde estou.

A meus queridos pais: Carlos e Natalia pelo seu amor não mensurável, apoio constante e pelo exemplo de vida.

A Igor Mencattini, meu orientador. Primeiro, por ter me orientado (ouvindo e tirando minhas dúvidas, explicando alguns dethales relacionados a esta dissertação que me ajudaram a entender melhor) e ter me apresentado esta área da matemática (Espaços de Teichmüller). Segundo, pela sua amizade e as conversas motivacionais e interessantes.

A os matemáticos que trabalharam e continuam trabalhando esta área da matemática e compartem suas pesquisas mediante seus artigos e livros os quais ajudam muito a estudantes como eu.

A os professores do departamento de Matemática do ICMC-USP pelos ensinamentos compartidos.

A companheiros estudantes do ICMC-USP (brasileiros, peruanos,...) que fazem de estudar e vivir em Brasil um grande prazer para mim.

À CAPES, Coordenação de Aperfeiçoamento de Pessoal de Nível Superior, pelo apoio financeiro sem o qual não teria sido possível desenvolver esta dissertação. 



\section{Resumo}

Nesta disertação estudamos as algumas ferramentas básicas relacionadas aos espaços de Teichmüller. Introduzimos o espaço de Teichmüller de gênero $g \geq 1$, denotado por $T_{g}$. O objetivo principal é construir as coordenadas de Fenchel-Nielsen $\omega_{G}: T_{g} \rightarrow \mathbb{R}_{+}^{3 g-3} \times \mathbb{R}^{3 g-3}$ para cada grafo cúbico marcado $G$. 
viii 


\section{Abstract}

In this dissertation we study the basic some tools related to Teichmüller space. We introduce the Teichmüller space of genus $g \geq 1$, denoted by $T_{g}$. The main goal is to construct the Fenchel-Nielsen coordinates $\omega_{G}: T_{g} \rightarrow \mathbb{R}_{+}^{3 g-3} \times \mathbb{R}^{3 g-3}$ to each marked cubic graph $G$. 


\section{Sumário}

\begin{tabular}{ll}
\hline Introdução & 1
\end{tabular}

1 Preliminares 3

1.1 Definições e resultados básicos . . . . . . . . . . . . . . . . 3

1.2 Geometria Hiperbólica . . . . . . . . . . . . . . . . . . . . . . . . 9 9

1.3 Grupos Fuchsianos . . . . . . . . . . . . . . . . . . . . . . 17

1.4 Regiões Fundamentais . . . . . . . . . . . . . . . . . . 26

2 Superfícies de Riemann 33

2.1 Definição e Exemplos . . . . . . . . . . . . . . . . . . . . . . . 33

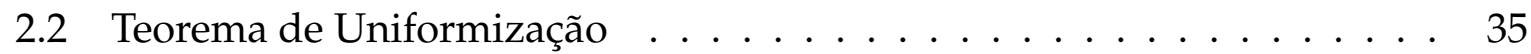

2.3 O quociente $\mathbb{H} / \Gamma \ldots \ldots \ldots \ldots \ldots$. . . . . . . . . . . . . . . 44

2.4 Métrica hiperbólica em superfícies de Riemann . . . . . . . . . . . . . . . 49

3 Espaço de Teichmüller $\quad 51$

$3.1 \quad$ Espaço de Teichmüller de gênero g . . . . . . . . . . . . . . . . . 51

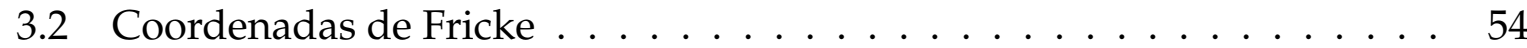

3.3 Estrutura conforme . . . . . . . . . . . . . . . . . . . . . 57

4 Coordenadas de Fenchel-Nielsen $\quad 61$

$4.1 \quad$ Estruturas hiperbólicas: Definições e resultados . . . . . . . . . . . . . 61

$4.1 .1 \quad Y$-peças e $X$-peças . . . . . . . . . . . . . . . 65

4.1 .2 O caso da assinatura $(1,1) \ldots \ldots \ldots \ldots$. . . . . . . . . . 73

4.1 .3 Grafos . . . . . . . . . . . . . . . . . . . . . . . . 74

4.2 Coordenadas de Fenchel-Nielsen . . . . . . . . . . . . . . . . 77

\begin{tabular}{|l|l}
\hline Referências Bibliográficas & 87
\end{tabular} 


\section{Introdução}

A teoria dos espaços de Teichmüller estuda as diferentes estruturas complexas em uma superfície dada. O problema de como parametrizar as variações destas estruturas complexas começou com Bernhard Riemann.

Uma superfície de Riemann é uma variedade complexa e conexa de dimensão 1. Diz-se que duas superficies de Riemann $R_{1}$ e $R_{2}$ têm a mesma estrutura complexa se existe um biholomorfismo de $R_{1}$ sobre $R_{2}$. Então, quantas estruturas complexas diferentes podem-se atribuir a uma variedade diferenciável orientada de dimensão 2 ?. Este problema é conhecido como o problema do moduli de Riemann.

O espaço de moduli $\mathcal{M}_{g}$ de gênero $g$, é o espaço das classes de superfícies de Riemann de gênero $g$ biholomorficamente equivalentes. Em seu artigo de 1857, "Theorie der Abel'schen Functionen", Riemann afirmou que $\mathcal{M}_{g}, g \geq 2$ é parametrizado por $3 g-3$ parâmetros complexos.

Em seu artigo de 1939, "Extremale quasikonforme Abildungen", Oswald Teichmüller determinou que o espaço $\mathcal{M}_{g}$ é identificado com o espaço quociente $T_{g} / \operatorname{Mod}_{g}$, onde $T_{g}$ é o espaço de Teichmüller de gênero $g$ e $M o d_{g}$ é um grupo discreto de automorfismos de $T_{g}$ chamado o grupo modular de Teichmüller.

No espaço $T_{g}$ existem as chamadas "coordenadas de Fenchel-Nielsen". É o objetivo desta dissertação apresentar estas coordenadas de $T_{g}$.

Esta dissertação esta dividido em 4 Capítulos, cujo contido de cada capítulo é descrito brevemente.

O Capítulo 1 esta dedicado a introduzir os conceitos e resultados relacionados à geometria hiperbólica (geometria não Euclideana que não satisfaz o postulado das paralelas) e aos grupos Fuchsianos, subgrupos discretos das isometrias do plano hiperbólico.

O Capítulo 2 esta dedicado às superfícies de Riemann (estruturas complexas de dimensiona 1 em uma variedade topológica). Mostramos que toda superfície de Riemann tem um recobrimento universal. Enunciamos o Teorema de Uniformização o 
qual classifica as superfícies de Riemann simplesmente conexas a menos de biholomorfismos e consequentemente classificamos as possíveis superfícies de Riemann a menos de biholomorfismos. Depois, estudamos algumas propriedades das superfícies de Riemann da forma $\mathbb{H} / \Gamma$, quociente do semiplano superior por um grupo Fuchsiano.

No Capítulo 3 introduzimos os espaços de Teichmüller $T_{g}$ de gênero $g$. Calculamos $T_{1}$ e para $T_{g}, \geq 2$ o objetivo é construir o espaço de Fricke $F_{g}$ o qual representa $T_{g}$ como um subconjunto de $\mathbb{R}^{6 g-6}$, para isto utilizamos resultados do Capítulos 1 e 2 . Por último, revisamos a equivalência entre os conceitos de estruturas complexas e estruturas conformes no caso 2-dimensional.

O Capítulo 4 é a parte principal desta dissertação. Primeiro revisamos o conceito de estrutura hiperbólica em superfícies e vemos que este conceito é equivalente ao conceito de superfícies de Riemann de gênero $g \geq 2$. Isto nos permite construir $T_{g}$ de uma maneira equivalente. Utilizando o fato de que toda superfície de Riemann de gênero $g \geq 2$ pode-se decompor em $2 g-2$ calças por meio de $3 g-3$ geodésicas fechadas simples introduzimos as coordenadas de Fenchel-Nielsen.

Resaltamos aqui que os gráficos desta dissertação foram tomados de:

[3] (Figuras 1.4, 1.5, 1.6, 4.1, 4.1, 4.1, 4.2, 4.3, 4.4, 4.5, 4.6, 4.7).

[7] (Figura 1.1).

[10] (Figuras 1.2, 1.3, 1.7, 2.1). 


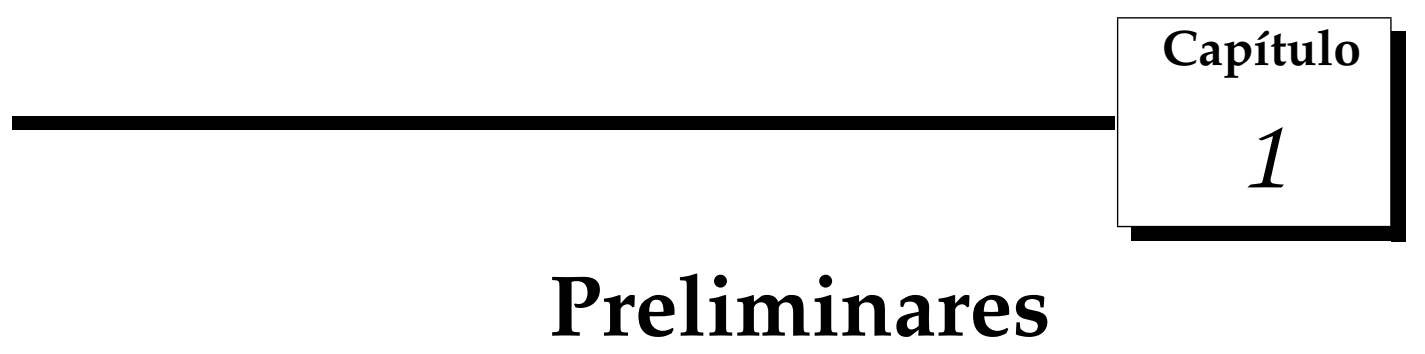

A partir deste capítulo e os demais capítulos denotaremos por $\mathbb{R}$ e $\mathbb{C}$ a reta real e o plano complexo, respectivamente. Se $z=x+i y \in \mathbb{C}$, escrevemos por $\operatorname{Re}(z)=x$ a parte real de $z$ e $\operatorname{Im}(z)=y$ a parte imaginária de $z$.

\subsection{Definições e resultados básicos}

Para começar, vamos apresentar a generalização natural de uma superfície Euclideana, a saber, as variedades diferenciáveis. Excelentes referências para este tema são [2], [6] e [12].

Definição 1.1. Um espaço de Hausdorff $S$ com base enumerável é uma variedade topológica $n$-dimensional, se para cada ponto $s \in S$, existe uma vizinhança $U$ homeomofa a um subconjunto aberto de $\mathbb{R}^{n}$. No caso $n=2$, dizemos que $S$ é uma superfície.

Assim, para qualquer variedade topológica $n$-dimensional $S$ existe família $\mathcal{A}=$ $\left\{\left(U_{i}, \varphi_{i}\right)\right\}_{i \in I},\left\{U_{i}\right\}_{i \in I}$ cobertura de $S$, chamado atlas de $S$; se $s \in U_{i}$ dizemos que $\left(U_{i}, \varphi_{i}\right)$ é uma carta (carta local ou sistema de coordenadas) em $s$ e $z_{i}=\varphi_{i}(s)$ é uma coordenada local para $s$.

Se $\left(U_{i}, \varphi_{i}\right)$ e $\left(U_{j}, \varphi_{j}\right)$ são cartas em $s \in S$ com coordenadas locais $z_{i}$ e $z_{j}$ para $s$, então $z_{i}=\left(\varphi_{i} \circ \varphi_{j}^{-1}\right)\left(z_{j}\right)$ expressa a mudança em coordenadas locais para $s$ correspondentes a duas cartas diferentes. Os homeomorfismos

$$
\varphi_{i} \circ \varphi_{j}^{-1}: \varphi_{j}\left(U_{i} \cap U_{j}\right) \rightarrow \varphi_{j}\left(U_{i} \cap U_{j}\right)
$$

são chamados mudanças de coordenadas. 
Definição 1.2. Um atlas $\mathcal{A}$ em uma variedade topologica $n$-dimensional $S$ é $C^{k}$-diferenciável (ou $C^{k}$ ), denotaado por $\mathcal{A} \in C^{k}, 1 \leq k \leq \infty$, se as mudanças de coordenadas são diferenciáveis de classe $C^{k}$.

Sejam $\mathcal{A}=\left\{\left(U_{i}, \varphi_{i}\right)\right\}_{i \in I}$ e $\mathcal{B}=\left\{\left(V_{j}, \psi_{j}\right)\right\}_{j \in J}$ dois atlas $C^{k}$ em $S$, dizemos que $\mathcal{A}$ e $\mathcal{B}$ são compativéis se $\mathcal{A} \cup \mathcal{B}$ é um atlas $C^{k}$ em $S$. Temos que a relação de compatibilidade é uma relação de equivalência.

Definição 1.3. Uma estrutura diferenciável de classe $C^{k}$ de dimensão $n$ em uma variedade topológica n-dimensional $M$ é uma classe de equivalência de atlas $C^{k}$ em $M$ e $M$ é chamado uma variedade diferenciável de classe $C^{k}$ ou simplesmente variedade diferenciável $C^{k}$ (superfície diferenciável ou simplesmente superfície no caso $n=2$ ). Dizemos que $M$ é uma variedade suave, se $M$ é uma variedade diferenciável $C^{\infty}$. Denotamos por $M^{n}$ para indicar que $M$ é uma variedade diferenciável n-dimensional.

Definição 1.4. Sejam $M^{m}$ e $N^{n}$ duas variedades diferenciáveis diferenciável $C^{k}$. Uma aplicação $f: M \rightarrow N$ é dita diferenciável (diferenciável de classe $C^{k}$ ) em $p \in M$, se existem cartas locais $x: U \rightarrow \mathbb{R}^{m}$ e $y: V \rightarrow \mathbb{R}^{n}$ com $p \in U$ e $f(U) \subset V$ tal que $y \circ f \circ x^{-1}: x(U) \rightarrow y(V)$ é diferenciável (diferenciável de classe $C^{k}$ ) em $x(p)$. Se f é diferenciável (diferenciável de classe $C^{k}$ ) em cada ponto de $M$, dizemos que $f$ é diferenciável (diferenciável de classe $C^{k}$ ou $C^{k}$ diferenciável) em $M$. Dizemos que fé suave se $f$ é $C^{\infty}$-diferenciável.

A continuação definimos o espaço tangente de $M^{m}$ em um ponto, existem outras maneiras equivalentes a esta para poder definir o espaço tangente.

Definição 1.5. Seja $M^{m}$ uma variedade suave. O espaço tangente a $M$ em $p \in M$ é definido como conjunto quociente $T_{p} M:=C_{p} M / \sim$, onde $C_{p} M=\{\lambda:(-\varepsilon, \varepsilon) \rightarrow M \mid \lambda(0)=$ $p$, $\lambda$ e diferenciável em 0$\}$ e a relação de equivalência $\sim$ é dado por: $\lambda \sim \mu$ se existe uma carta local $x: U \rightarrow \mathbb{R}^{m}$ em $M$ com $p \in U$ tal que $(x \circ \lambda)^{\prime}(0)=(x \circ \mu)^{\prime}(0)$. Os elementos de $T_{p} M$ são chamados vetores tangentes. Denotamos por $[\lambda]$ (ou $\left.\lambda^{\prime}\right)$ a classe de equivalência de $\lambda \in C_{p} M$.

Observação 1.6. 1.- Se $M^{m}$ é uma variedade suave, então $T_{p} M$ é um espaço vetorial real de dimensão $m$. Com efeito, dado qualquer carta local $(U, x)$ em $M \operatorname{com} p \in M$, temos uma bijeção $\bar{x}: T_{p} M \rightarrow \mathbb{R}^{m}$ dada por $\lambda^{\prime} \mapsto(x \circ \lambda)^{\prime}(0)$ e a estrutura de espaço vetorial em $T_{p} M$ é de tal forma tal que $\bar{x}$ se torne um isomorfismo de espaços vetoriais. 2.- Consideremos o conjunto $B=\left\{\frac{\partial}{\partial x_{i}}\right\}_{i=1}^{m}$ tal que $\bar{x}\left(\frac{\partial}{\partial x_{i}}\right)=e_{i}$ ( $i$-ésimo vetor canônico de $\mathbb{R}^{m}$ ), então $B$ é uma base de $T_{p} M$.

Definição 1.7. Seja $f: M \rightarrow N$ uma aplicação diferenciável em $p \in M$. O diferencial de $f$ em $p$, é a aplicação linear $d f_{p}: T_{p} M \rightarrow T_{f(p)} N$ dada por $\lambda^{\prime} \mapsto(f \circ \lambda)^{\prime}$. Se $f$ é suave em $M$, 
dizemos que f é uma:

(i) imersão se $d f_{p}$ é injetora para todo $p \in M$,

(ii) mergulho se $f$ é uma imersão e $f$ é um homeomorfismo de $M$ sobre sua imagem $f(M)$ (com a topologia induzida de $N$ ).

(iii) submersão se $d f_{p}$ é sobrejetora para todo $p \in M$.

Definição 1.8. Uma métrica Riemanniana em uma variedade suave n-dimensional $M$ é uma correspondência g que associa a cada ponto $p \in M$, um produto interno $g_{p}: T_{p} M \times T_{p} M \rightarrow \mathbb{R}$. Dizemos que gédiferenciável, se para toda carta local $(U, x)$ em $M$ as funções $g_{i j}^{x}: U \rightarrow \mathbb{R}$ dado por $g_{i j}^{x}(p)=g_{p}\left(\frac{\partial}{\partial x_{i}}(p), \frac{\partial}{\partial x_{j}}(p)\right)$ são diferenciavéis para todo $i, j=1, \ldots, n$. Uma variedade Riemanniana $M$ é uma variedade suave $M$ munida com uma métrica Riemanniana suave $g$.

Proposição 1.9. Ver [2, pág.116]. Toda variedade suave $M$ tem pelo menos uma métrica Riemanniana suave.

Observação 1.10. Se $\left(M^{m}, g\right)$ é uma variedade Riemanniana e $x: U \rightarrow \mathbb{R}^{m}$ é uma carta local, as funções $x_{i}: U \rightarrow \mathbb{R}, i=1, \ldots, m$ originam para todo $p \in U$ a 1-forma $\left(d x_{i}\right)_{p}$ : $T_{p} M \rightarrow \mathbb{R}$ dado por $\frac{\partial}{\partial x_{j}} \mapsto \delta_{i j}$. O produto tensorial das 1 -formas $\left(d x_{i}\right)_{p}$ e $\left(d x_{j}\right)_{p}$ é a aplicação bilinear $\left(d x_{i}\right)_{p} \otimes\left(d x_{j}\right)_{p}: T_{p} M \times T_{p} M \rightarrow \mathbb{R}$ dado por $(v, w) \mapsto\left(d x_{i}\right)_{p}(v)\left(d x_{j}\right)_{p}(w)$ e o produto simétrico $\left(d x_{i}\right)_{p}\left(d x_{j}\right)_{p}=\frac{1}{2}\left(\left(d x_{i}\right)_{p} \otimes\left(d x_{j}\right)_{p}+\left(d x_{j}\right)_{p} \otimes\left(d x_{i}\right)_{p}\right)$ e denotamos por $\left(d x_{i}\right)_{p}^{2}$ a $\left(d x_{i}\right)_{p}\left(d x_{i}\right)_{p}$.

Nestas condições pode-se ver que $g$ se expressa na forma

$$
g=\sum_{i, j=1}^{m} g_{i j}^{x} d x_{i} d x_{j}
$$

Exemplo 1.11. A métrica Euclideana em $\mathbb{R}^{m}$. Neste caso $g_{i j}=\delta_{i j}$ e $g=\sum_{i=1}^{m} d x_{i}^{2}$.

Definição 1.12. Seja $M$ uma variedade suave e $(N, h)$ uma variedade Riemanniana. Dada uma imersão $f: M \rightarrow N$ definimos na variedade $M$ a métrica Riemanniana $g$ dada por $g_{p}(v, w):=h_{f(p)}\left(d f_{p}(v), d f_{p}(w)\right)$ para cada $p \in M$ e $v, w \in T_{p} M$. Tal métrica é chamada a métrica induzida por $f$ em $M$ ou métrica pullback denotada por $g=f^{*} h$.

Definição 1.13. Sejam $\left(M^{m}, g\right)$ e $\left(N^{n}, h\right)$ duas variedades Riemannianas e seja $f: M \rightarrow N$ uma aplicação suave tal que $g_{p}(v, w)=h_{f(p)}\left(d f_{p} v, d f_{p} w\right)$, para todo $p \in M$ e $v, w \in T_{p} M$, então $f$ é chamada uma imersão isómétrica (isto implica $m \leq n$ ). No caso que $m=n$, $f$ é chamada isometria local e se além disso tal $f$ é injetora, então $f$ é chamada de isometria e dizemos que $M$ eN são isómetricos.

Agora, vamos apresentar algumas definições e resultados topológicos que serão utilizados posteriormente. 
Seja $X$ um espaço topológico. Um caminho em $X$ é uma função contínua $\alpha: \mathrm{I} \rightarrow X$, onde I é algum intervalo fechado de $\mathbb{R}$ que geralmente consideraremos o intervalo $[0,1]$. Os pontos $\alpha(0)$ e $\alpha(1)$ são chamados pontos inicial e final. O caminho $\alpha$ é chamado fechado ou laço se $\alpha(0)=\alpha(1)$. Para dois caminhos $\alpha$ e $\beta$ em $X$ tais que $\alpha(1)=\beta(0)$, definimos o caminho produto

$$
\alpha \cdot \beta(x)= \begin{cases}\alpha(x) & \text { si } x \in\left[0, \frac{1}{2}\right] \\ \beta(x) & \text { si } x \in\left[\frac{1}{2}, 1\right]\end{cases}
$$

Definição 1.14. Seja $X$ um espaço topológico. Dois caminhos $\alpha$ e $\beta$ em $X$ são ditos homotópicos, denotado por $\alpha \simeq \beta$, se eles têm o mesmo ponto inicial $x_{0}$ e o mesmo ponto final $x_{1}$ e se existe uma aplicação contínua $F:[0,1] \times[0,1] \rightarrow X$ tal que

$$
\begin{array}{ccc}
F(t, 0)=\alpha(t) & F(t, 1)=\beta(t) & 0 \leq t \leq 1 \\
F(0, s)=x_{0} & F(1, s)=x_{1} & 0 \leq s \leq 1 .
\end{array}
$$

Tal F é chamada homotopia entre $\alpha$ e $\beta . \simeq$ é uma relação de equivalência.

Definição 1.15. Seja $X$ um espaço topológico e $x_{0}$ um ponto fixado de $X$. O grupo fundamental de $X$ com ponto base em $x_{0}$, denotado por $\pi_{1}\left(X, x_{0}\right)$, é o conjunto das classes de homotopia de laços baseados em $x_{0}$ com a operação $[\alpha] \cdot[\beta] \mapsto[\alpha \cdot \beta]$.

Definição 1.16. (i) Dois laços $\alpha, \beta:[0,1] \rightarrow X$ são livremente homotópicos se existe uma aplicação contínua $F:[0,1] \times[0,1] \rightarrow X$ tal que

$$
F(t, 0)=\alpha(t) \quad F(t, 1)=\beta(t) \quad F(0, s)=F(1, s) \quad \forall t, s \in[0,1] .
$$

Denotamos por $\tilde{\Pi}_{1}(X)$, o conjunto das classes de laços livremente homotópicos em $X$.

(ii) Sejam A e B subconjuntos fechados e assumamos que c, $\gamma:[a, b] \rightarrow S$ curvas com pontos iniciais $c(a), \gamma(a) \in A$ e pontos finais $c(b), \gamma(b) \in B$. Dizemos que cé homotópica a $\gamma$ com pontos extremos em $A$ e $B$ se existe uma aplicação contínua $\phi:[a, b] \times[0,1] \rightarrow X$ tal que

$$
\begin{aligned}
& \phi(t, 0)=c(t) \quad \phi(t, 1)=\gamma(t) \quad a \leq t \leq b, \\
& \phi(a, s) \in A \quad \phi(b, s) \in B \quad 0 \leq s \leq 1 .
\end{aligned}
$$

Definição 1.17. Sejam $f, g: X \rightarrow Y$ duas aplicações contínuas entre espaços topológicos $X e$ $Y$. Dizemos que $f$ e $g$ são homotópicos, se existe uma aplicação contínua $F: X \times[0,1] \rightarrow Y$ tal que $F(\cdot, 0)=f$ e $F(\cdot, 1)=g$.

Definição 1.18. Dois homeomorfismos $\phi_{0}, \phi_{1}: A \rightarrow B$ de espaços topológicos $A, B$ são ditos 
isotópicos se existe uma aplicação contínua $J: A \times[0,1] \rightarrow B$ tak que $J(\cdot, 0)=\phi_{0}, J(\cdot, 1)=$ $\phi_{1}$ e $J(\cdot, s): A \rightarrow B$ é um homeomorfismo para cada $s \in[0,1]$. Um homeomorfismo $h: A \rightarrow A$ o qual é isotópico a identidade é chamado um 1-homeomorfismo.

O seguinte Teorema (Ver [11, pág.26]) é um resultado importante e conhecido de topología que classifica todas as superfíces sem bordo, compactas, conexas e orientavéis a menos de homeomorfismo.

Teorema 1.19. Cada superfície $S$ orientável sem bordo, compacta e conexa é homeomorfa a uma superfície $S_{g}$ obtida por adjuntar $g \geq 0$ alças a uma esfera (Ver Figura 1.1. $g=3$ ). Tal único número gé chamado o gênero da superfície $S$

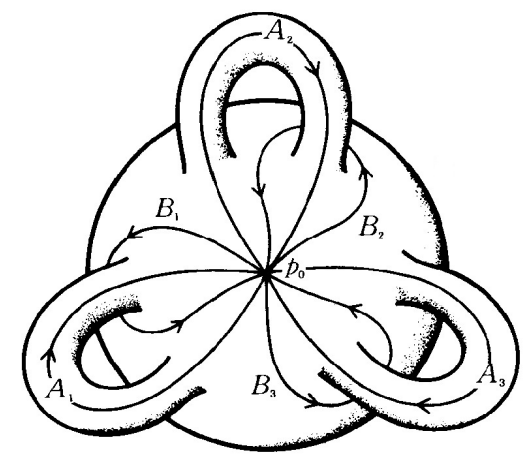

Figura 1.1: Superfície compacta, sem fronteira de gênero 3

Tomando um ponto $p_{0}$ em algum $S_{g}$, cortamos $S_{g}$ ao longo de laços simples $A_{1}, B_{1}$, $\ldots, A_{g}, B_{g}$ com ponto base em $p_{0}$ como na Figura 1.1. Então, obtemos um domínio homeomorfo a um polígono convexo com $4 g$ lados. O grupo fundamental $\pi_{1}\left(S_{g}, p_{0}\right)$ é gerado pelas classes de homotopia $\left[A_{1}\right],\left[B_{1}\right], \ldots,\left[A_{g}\right],\left[B_{g}\right]$ (Ver [11, pág.99]) induzidas de $A_{1}, B_{1}, \ldots, A_{g}, B_{g}$ e satisfaz a relação fundamental

$$
\prod_{j=1}^{g}\left[A_{j}\right] \cdot\left[B_{j}\right] \cdot\left[A_{j}\right]^{-1} \cdot\left[B_{j}\right]^{-1}=\left[p_{0}\right]
$$

Chamamos a $\left\{\left[A_{j}\right],\left[B_{j}\right]\right\}_{j=1}^{g}$ ou a $\left\{A_{j}, B_{j}\right\}$ um sistema canônico de geradores de $\pi_{1}\left(S_{g}, p_{0}\right)$. O seguinte resultado pode-se encontrar em [4, pág.44].

Teorema 1.20. Seja $S$ uma superfície compacta suave. Então, todo homeomorfismo de $S$ é isotópico a um difeomorfismo de $S$.

Definição 1.21. Uma subdivisão poligonal $M$ de uma superfície $S$ consiste de um conjunto finito de pontos de $S$ chamados vértices e um conjunto finitos de caminhos simples em $S$ chamados arestas tal que: 
(i) Toda aresta tem dois pontos extremos, tal que tais pontos são vértices.

(ii) As arestas podem-se intersectar somente em seus pontos extremos.

(iii) A união das arestas (a qual também denotamos por $M$ ) é conexo.

(iv) As componentes de $S \backslash M$ são homeomorfos a discos abertos.

Tais componentes são chamados faces.

Definição 1.22. A característica de Euler de uma superfície S compacta e conexa é definido como

$$
\chi(S)=\chi(M)=V-E+F
$$

onde $M$ é uma subdivisão poligonal de $S$ com $V$ vértices, $E$ arestas e $F$ faces.

O seguinte resultado pode-se encontrar em [8, pág.196]

Teorema 1.23. (i) Se S é uma superfície compacta e conexa, então $\chi(S)$ é bem definida, isto é, não depende da subdivisão poligonal $M$ de $S$.

(ii) Se Sé orientável de gênero $g$, então $\chi(S)=2-2 g$.

Por último, vamos apresentar algumas definições e notações relacionadas com as transformações de Möbius as quais serão utilizados posteriormenente.

Definição 1.24. 1.- Uma transformação de Möbius é uma aplicação da forma

$$
T(z)=\frac{a z+b}{c z+d} \quad a d-b c \neq 0, a, b, c, d \in \mathbb{C} .
$$

2.- $\operatorname{PSL}(2, \mathbb{C})$ é o conjunto de todas as transformações de Möbius tal que ad - bc $=1$.

3.- $P S L(2, \mathbb{R})$ é o subgrupo de $P S L(2, \mathbb{C})$ tal que os coeficientes $a, b, c, d \in \mathbb{R}$.

4.- O grupo modular $P S L(2, \mathbb{Z})$ é o subgrupo de $P S L(2, \mathbb{R})$, tal que os coeficientes a, $b, c, d \in$ $\mathbb{Z}$.

Observação 1.25. Existe uma identificação canônica entre $S L(2, \mathbb{R}) /\{ \pm I\}$ e $P S L(2, \mathbb{R})$, pois se consideramos $M: S L(2, \mathbb{R}) \rightarrow P S L(2, \mathbb{R})$ dado por $M(A)(z)=(a z+b) /(c z+d)$ onde

$$
A=\left[\begin{array}{ll}
a & b \\
c & d
\end{array}\right] \in S L(2, \mathbb{R})
$$

tem-se que $M$ é um homomorfismo sobrejetivo com núcleo $\pm I$. Analogamente temos uma identificação para $P S L(2, \mathbb{C})$ e $P S L(2, \mathbb{Z})$.

Por ultimo, lembrar que a razão cruzada de quatro pontos diferentes $z_{1}, z_{2}, z_{3}, z_{4} \in$ $\mathbb{C} \cup\{\infty\}$ é definido pela fórmula

$$
\left(z_{1}, z_{2} ; z_{3}, z_{4}\right)=\frac{\left(z_{1}-z_{2}\right)\left(z_{3}-z_{4}\right)}{\left(z_{2}-z_{3}\right)\left(z_{4}-z_{1}\right)}
$$




\subsection{Geometria Hiperbólica}

Nesta seção apresentamos uma variedade Riemanniana $(\mathbb{H}, g)$ chamada o plano hiperbólico e veremos algumas de suas propiedades. Uma aplicação importante da geometria hiperbólica é que nos permite estudar a geometria das superfícies de dimensão 2. As principais referências para esta seção são [1], [8], [10].

Definição 1.26. O modelo de Poincaré do plano hiperbólico é a variedade Riemanniana $\left(\mathbb{H}, d s^{2}\right)$, onde $\mathbb{H}=\left\{(x, y) \in \mathbb{R}^{2} \mid y>0\right\}$ (semiplano superior de $\mathbb{R}^{2}$ ) tem a estrutura diferenciável suave induzida de $\mathbb{R}^{2}$ e ds $s^{2}$ é a métrica de Poincaré (métrica hiperbólica) definida por

$$
d s^{2}=\frac{d x^{2}+d y^{2}}{y^{2}}=\frac{|d z|^{2}}{y^{2}} \quad(z=x+i y)
$$

Em termos de variável complexa, $\mathbb{H}$ é o conjunto $\{z \in \mathbb{C} \mid \operatorname{Im}(z)>0\}$ e a métrica é dada por

$$
d s^{2}=-\frac{4 d z d \bar{z}}{(z-\bar{z})^{2}}
$$

O biholomorfismo $z \mapsto \frac{z-i}{z+i}$ mapea biholomorficamente $\mathbb{H}$ sobre $\Delta=\{z \in \mathbb{C}|| z \mid<1\}$, o disco unitário. A métrica induzida por esta aplicação em $\Delta$ é

$$
d s^{2}=\frac{4 d z d \bar{z}}{\left(1-|z|^{2}\right)^{2}}=\frac{4\left(d x^{2}+d y^{2}\right)}{\left(1-\left(x^{2}+y^{2}\right)\right)^{2}}=\quad z=x+i y \in \Delta .
$$

Este é o modelo do disco do plano hiperbólico.

Por construção os modelos de Poincaré e do disco são isométricos.

Observação 1.27. 1.- Dado uma métrica Riemanniana $g$ da forma $g=h(x, y)^{2}\left(d x^{2}+d y^{2}\right)$ onde $h: \Omega \subset \mathbb{R}^{2} \rightarrow \mathbb{R}_{+}$define-se a curvatura Gaussiana de $h$ dado por

$$
K(g)=-\frac{4}{h^{2}}\left(\frac{\partial^{2}}{\partial x^{2}}+\frac{\partial^{2}}{\partial y^{2}}\right)(\ln h)
$$

Então, fazendo os cálculos, podemos ver que a curvatura Gaussiana da métrica de Poincaré tem curvatura Gaussiana constante igual a -1 em $\mathbb{H}$.

2.- A curvatura Gaussiana é um invariante sob isometrias locais. Para uma prova deste resultado, assim como a definição geral da curvatura Gaussiana para variedades de dimensão maior, o leitor pode revisar [2, Capítulo 5].

3.- Em uma variedade Riemanniana, a métrica Riemanniana é chamada hiperbólica, se ela tem curvatura Gaussiana constante -1 . 
Tem-se uma ação natural de $P S L(2, \mathbb{R})$ em $\mathbb{H}$ com as seguintes propriedades.

Teorema 1.28. Ver [8, pág.218].

(i) $P S L(2, \mathbb{R})$ é transitiva em $\mathbb{H}$.

(ii) $\operatorname{PSL}(2, \mathbb{R})$ é duplamente transitiva em $\mathbb{H}$ (isto é, dados $\left(\alpha_{1}, \alpha_{2}\right), \alpha_{1} \neq \alpha_{2}$ e $\left(\beta_{1}, \beta_{2}\right), \beta_{1} \neq$ $\beta_{2}$ com $\alpha_{i}, \beta_{i} \in \mathbb{H}, i=1,2$ então existe $g \in P S L(2, \mathbb{R})$ tal que $\left.g\left(\alpha_{j}\right)=\beta_{j}, j=1,2\right)$.

Definição 1.29. Seja $\gamma: I \rightarrow \mathbb{H}$ um caminho diferenciável por partes dado por $\gamma(t)=x(t)+$ $i y(t)$. Definimos o comprimento hiperbólico ( $\mathbb{H}$-comprimento) de $\gamma$, denotado por $h(\gamma)$, por

$$
h(\gamma)=\int_{0}^{1} \frac{\sqrt{\left(\frac{d x}{d t}\right)^{2}+\left(\frac{d y}{d t}\right)^{2}}}{y(t)} d t=\int_{0}^{1} \frac{\left|\frac{d \gamma}{d t}\right|}{y(t)} d t
$$

Proposição 1.30. O comprimento hiperbólico $h(\gamma)$ é invariante sob $P S L(2, \mathbb{R})$, isto é, $h(T(\gamma))=$ $h(\gamma)$ para todo $T \in P S L(2, \mathbb{R})$.

Prova: Seja $T(z)=\frac{a z+b}{c z+d} \in P S L(2, \mathbb{R})$, se $w(t)=T(\gamma(t))=u(t)+i v(t)$, temos que $\frac{d w}{d z}=\frac{1}{(c z+d)^{2}}$ e $v=\frac{y}{|c z+d|^{2}}$ e assim $\left|\frac{d w}{d z}\right|=\frac{v}{y}$. Portanto

$$
h(T(\gamma))=\int_{0}^{1} \frac{\left|\frac{d w}{d t}\right|}{v(t)} d t=\int_{0}^{1} \frac{\left|\frac{d w}{d z} \frac{d z}{d t}\right|}{v(t)} d t=\int_{0}^{1} \frac{\left|\frac{d z}{d t}\right|}{y(t)} d t
$$

Agora, vamos mostrar que entre dois pontos quaisquer de $\mathbb{H}$ existe um único caminho de menor comprimento hiperbólico juntando tais pontos. Tais caminhos serão chamados segmentos geodésicos ou arcos geodésicos.

Teorema 1.31. Os segmentos geodésicas em $\mathbb{H}$ são arcos de semi-círculos com centro no eixo real e os segmentos de retas perpendiculares ao eixo real.

Prova: Sejam $z_{1}$ e $z_{2}$ dois pontos em $\mathbb{H}$. Suponhamos primeiro que $z_{1}=i a$ e $z_{2}=$ $i b(b>a)$. Se $\gamma: I \rightarrow \mathbb{H}$ é um caminho diferenciável por partes juntando $i a$ e $i b$, com $\gamma(t)=(x(t), y(t))$, então

$$
h(\gamma)=\int_{0}^{1} \frac{\sqrt{\left(\frac{d x}{d t}\right)^{2}+\left(\frac{d y}{d t}\right)^{2}}}{y(t)} d t \geq \int_{0}^{1} \frac{\left|\frac{d y}{d t}\right|}{y(t)} d t \geq \int_{0}^{1} \frac{\frac{d y}{d t}}{y(t)} d t=\int_{a}^{b} \frac{d y}{y}=\ln \frac{b}{a} .
$$

A igualdade se cumpre se e somente se $d x / d t=0$ e $d y / d t \geq 0$. Como $\gamma$ é diferenciável por partes, temos que $\gamma$ é o segmento de reta Euclideana juntando $i a$ e $i b$.

Fazendo o mesmo cálculo, mostra que se $z_{1}$ e $z_{2}$ tem a mesma parte real, então o único segmento geodésico juntando estes pontos é o único segmento de reta Euclideana que 
os junta.

Agora suponhamos que $z_{1}$ e $z_{2}$ não têm a mesma parte real. Então o bisector perpendicular do segmento de reta Euclideano que os junta corta o eixo real em um ponto $r$ o qual é o centro do único círculo Euclideano $Q$ através $z_{1}$ e $z_{2}$ ortogonal a $\mathbb{R}$. Suponhamos que $Q$ intersecta $\mathbb{R}$ em $z_{1}^{*}, z_{2}^{*}$. Pelo Teorema $1.28(i i)$, existe $T \in P S L(2, \mathbb{R})$ tal que $T\left(z_{1}^{*}\right)=0, T\left(z_{2}^{*}\right)=\infty$. Logo, podemos afirmar que $T(Q)$ é o eixo imaginário tal que o segmento geodésico juntando $T\left(z_{1}\right)$ e $T\left(z_{2}\right)$ é o segmento do eixo imaginário que os junta. Daqui, por Teorema 1.30, existe um único segmento geodésico juntando $z_{1}$ e $z_{2}$, a saber, o arco de $Q$ em $\mathbb{H}$ juntando $z_{1}$ e $z_{2}$.

Os semi-círculos cujos centros estão no eixo real ou as semi-retas Euclideanas perpendiculares a $\mathbb{R}$ contidos em $\mathbb{H}$ serão chamados geodésicas ou $\mathbb{H}$-linhas. Consideramos as $\mathbb{H}$-linhas tendo um ponto extremo em $\infty$, assim toda $\mathbb{H}$-linha tem dois pontos extremos em $\mathbb{R} \cup\{\infty\}$.

Teorema 1.32. $P S L(2, \mathbb{R})$ age transitivamente no conjunto das $\mathbb{H}-$-linhas.

Prova: Sejam $Q, Q^{\prime}$ duas $\mathbb{H}$-linhas. Se $Q$ tem pontos extremos $s, t \in \mathbb{R} \cup\{\infty\}$ e $Q^{\prime}$ tem pontos extremos $s^{\prime}, t^{\prime} \in \mathbb{R} \cup\{\infty\}$ então por Teorema $1.28($ ii $)$ existe um $T \in P S L(2, \mathbb{R})$ tal que $T(s)=s^{\prime}, T(t)=t^{\prime}$ e é claro que os pontos extremos de uma $\mathbb{H}$-linha o determinam unicamente, portanto $T(Q)=Q^{\prime}$.

Definição 1.33. A distância hiperbólica $\rho(z, w)$ entre dois pontos $z, w \in \mathbb{H}$ é definida como $o \mathbb{H}$-comprimento do segmento da $\mathbb{H}$-linha que junta tais pontos.

Como consequência do Teorema 1.30 tem-se a seguinte Proposição.

Proposição 1.34. Qualquer elemento $T$ de $P S L(2, \mathbb{R})$ é uma isometria de $\mathbb{H}$, isto é, $\rho(T(z), T(w))=$ $\rho(z, w)$.

Agora apresentamos algumas fórmulas para poder calcular a distância hiperbólica entre dois pontos

Lema 1.35. Sejam $z, w \in \mathbb{H}$ e seja $Q$ a geodésica que passa por $z$ e $w$ intersectando-se com $\mathbb{R} \cup\{\infty\}$ em $z^{*}, w^{*}$, escolhidos de tal maneira que z esta entre $z^{*}$ e $w$. Então existe um único elemento $T \in P S L(2, \mathbb{R})$ tal que $T\left(z^{*}\right)=0, T\left(w^{*}\right)=\infty$ e $T(z)=i$. Também $T(w)=$ ri $(r>$ 1) $e \rho(z, w)=\ln r$

Prova: Assumamos que $z^{*}$ nem $w^{*}$ é $\infty$. Podemos supor que $z^{*}>w^{*}$. Se pomos $S(\zeta)=$ $\frac{\zeta-z^{*}}{\zeta-w^{*}}$ então $S \in P S L(2, \mathbb{R})$ e $S\left(z^{*}\right)=0, S\left(w^{*}\right)=\infty$, assim $S$ mapea $Q$ ao eixo imginário. Se $S(z)=k i$ então $T=U_{1 / k} \circ S, U_{\lambda}(z)=\lambda z, \lambda \neq 0$, 1 é a transformação requerida. A 
unicidade segue-se do fato que toda transformação de Möbius é unicamente determinada por três pontos. Como $z$ esta entre $z^{*}$ e $w, T(z)=i$ esta entre $T\left(z^{*}\right)=0$ e $T(w)$, $\operatorname{logo} T(w)=r i, r>1$. Portanto $\rho(z, w)=\rho(T(z), T(w))=\rho(i, r i)=\ln r$.

Lema 1.36. (i) Se $z, w, z^{*}, w^{*}$ são definidos como no Lema 1.35, então a razão cruzada $\eta(z, w)=$ $\left(w, z^{*} ; z, w^{*}\right)$ satisfaz $\eta(T(z), T(w))=\eta(z, w)$ para todo $T \in P S L(2, \mathbb{R})$.

(ii) Para todo $z, e \in \mathbb{H}$ a aplicação $\tau(z, w)=\left|\frac{z-w}{z-\bar{w}}\right|$ satisfaz $\tau(T(z), T(w))=\tau(z, w)$ para todo $T \in P S L(2, \mathbb{R})$.

Prova: (i) Como $P S L(2, \mathbb{R})$ preserva $\mathbb{H}$-linhas, a $\mathbb{H}$-linha juntando $T(z)$ e $T(w)$ tem pontos extremos $T\left(z^{*}\right)$ e $T\left(w^{*}\right)$ e o resultado segue-se da invariância da razão cruzada sob transformações de Möbius.

(ii) Essa igualdade segue-se da identidade $|T(z)-T(w)|=|z-w|\left|T^{\prime}(z) T^{\prime}(w)\right|^{1 / 2}$ para todo $T \in P S L(2, \mathbb{R})$.

Teorema 1.37. Para $z, w \in \mathbb{H}$ temos que:

(i) $\rho(z, w)=\ln \frac{|z-\bar{w}|+|z-w|}{|z-\bar{w}|-|z-w|}$

(ii) $\cosh \rho(z, w)=1+\frac{|z-w|^{2}}{2 \operatorname{Im}(z) \operatorname{Im}(w)}$

(iii) $\sinh \left[\frac{1}{2} \rho(z, w)\right]=\frac{|z-w|}{2(\operatorname{Im}(z) \operatorname{Im}(w))^{1 / 2}}$

(iv) $\cosh \left[\frac{1}{2} \rho(z, w)\right]=\frac{|z-\bar{w}|}{2(\operatorname{Im}(z) \operatorname{Im}(w))^{1 / 2}}$

Prova: Se $z, w, r$ são definidos como no Lema 1.36 , então $\eta(z, w)=(r i, 0 ; i, \infty)=r$ e portanto $\rho(z, w)=\ln \eta(z, w)$. Também

$$
\tau(z, w)=\tau(i, r i)=\frac{r-1}{r+1}=\frac{e^{\rho(z, w)}-1}{e^{\rho(z, w)}+1}
$$

e portanto $\rho(z, w)=\ln \frac{1+\tau(z, w)}{1-\tau(z, w)}$. Assim obtemos $(i)$.

Da identidade $\sinh ^{2} \frac{u}{2}=\frac{\tanh ^{2}(u / 2)}{1-\tanh ^{2}(u / 2)}$, obtemos

$$
\sinh ^{2} \frac{1}{2} \rho(z, w)=\frac{t(z, w)^{2}}{1-\tau(z, w)^{2}}=\frac{|z-w|^{2}}{|z-\bar{w}|^{2}-|z-w|^{2}}
$$

mas $|z-\bar{w}|^{2}-|z-w|^{2}=4 \operatorname{Im}(z) \operatorname{Im}(w)$. Portanto obtemos (iii). Os outros items podem-se obter de $(i i i)$.

A Proposição 1.34 diz que o conjunto de isometrias de $\mathbb{H}$, denotado por $\operatorname{Isom}(\mathbb{H})$, contém o grupo $\operatorname{PSL}(2, \mathbb{R})$. Agora vamos identificar todas as isometrias de $\mathbb{H}$. 
Proposição 1.38. I $\operatorname{som}(\mathbb{H})=P S L(2, \mathbb{R}) \cup\left\{\frac{a \bar{z}+b}{c \bar{z}+d} \mid a d-b c=-1, a, b, c, d \in \mathbb{R}\right\}$.

Prova: Seja $\phi$ uma isometria de $\mathbb{H}$, então $\phi$ mapea geodésicas em geodésicas. Se $I$ é o eixo imaginário, $\phi(I)$ é uma geodésica. Logo, pelo Teorema 1.32 , existe $g \in P S L(2, \mathbb{R})$ o qual mapea $\phi(I)$ a $I$. Aplicando as tranformações $z \mapsto k z,(k>0)$ e $z \mapsto \frac{-1}{z}$ podemos assumir que $g \circ \phi$ fixa $i$ e mapea $(i, \infty)$ e $(0, i)$ sobre eles mesmos, daqui $g \circ \phi$ fixa cada ponto de $I$.

Seja $z=x+i y \in \mathbb{H}$ e $g \circ \phi(z)=u+i v$. Para todo positivo $t$, temos

$$
\rho(z, i t)=\rho(g \circ \phi(z), g \circ \phi(i t))=\rho(u+i v, i t)
$$

e pelo Teorema 1.37 (iii) temos

$$
\left[x^{2}+(y-t)^{2}\right] v=\left[u^{2}+(v-t)^{2}\right] y
$$

Como isto cumpre-se para todo $t>0$, divindo ambos lados da equação por $t^{2} \mathrm{e}$ tomando $t \rightarrow \infty$, temos $v=y$, e $x^{2}=u^{2}$. Portanto $g \circ \phi(z)=z$ ou $-\bar{z}$. Como as isometrias são continuas, então somente um destes casos se cumpre para todo $z \in \mathbb{H}$. Se $g \circ \phi(z)=z$, então $\phi \in P S L(2, \mathbb{R})$. Se $g \circ \phi(z)=-\bar{z}$ temos que

$$
\phi(z)=\frac{a \bar{z}+b}{c \bar{z}+d} \quad a d-b c=-1 .
$$

Devido a esta proposição, temos a seguinte definição.

Definição 1.39. O conjunto das isometrias de $\mathbb{H}$ que preservam orientação será denotado por $I_{\text {som }}^{+}(\mathbb{H})$. Além disso o conjunto das isometrias de $\mathbb{H}$ que revertem orientação será denotado por $\operatorname{Isom}^{-}(\mathbb{H})$.

Definição 1.40. Seja $Q$ uma $\mathbb{H}$-linha. Então uma reflexão hiperbólica (HH-reflexão) em $Q$ é uma isometria de $\mathbb{H}$, diferente da identidade, o qual fixa todos os pontos de $Q$.

Observação 1.41. Se $I$ é o eixo imaginário, da Proposição 1.38, temos que $R_{0}: z \mapsto-\bar{z}$ é uma $\mathbb{H}$-reflexão. Se $Q$ é outra $\mathbb{H}$-linha, então pelo Teorema 1.32 existe $T \in P S L(2, \mathbb{R})$ tal que $T(Q)=I$, como $T$ é uma isometria, $T^{-1} R_{0} T$ é uma $\mathbb{H}$-reflexão em $Q$.

A seguinte proposição é uma consequência do Teorema 1.37 (pois a família de todos discos abertos hiperbólicos coincide com a família de discos abertos Euclideanos).

Proposição 1.42. A topologia induzida pela métrica hiperbólica é a mesma topologia pela métrica Euclideana 
Definição 1.43. Se $E \subset \mathbb{H}$ definimos $\mu(E)$, a área hiperbólica ( $\mathbb{H}$-área) de E, por

$$
\mu(E)=\iint_{E} \frac{d x d y}{y^{2}}
$$

se a integral existe.

Teorema 1.44. A $\mathbb{H}$-área é invariante sob $\operatorname{PS} L(2, \mathbb{R})$, isto é, $\mu(T(E))=\mu(E)$ para todo $T \in P S L(2, \mathbb{R})$.

Prova: Seja $T(z)=(a z+b) /(c z+d) \in p \operatorname{com} z=x+i y$, e $w=T(z)=u+i v$. Utilizando as equações de Cauchy-Riemann, temos que

$$
\frac{\partial(u, v)}{\partial(x, y)}=\frac{\partial u}{\partial x} \frac{\partial v}{\partial y}-\frac{\partial u}{\partial y} \frac{\partial v}{\partial x}=\left(\frac{\partial u}{\partial x}\right)^{2}+\left(\frac{\partial v}{\partial x}\right)^{2}=\left|\frac{d T}{d z}\right|^{2}=\frac{1}{|c z+d|^{4}}
$$

Assim

$$
\mu(T(E))=\iint_{T(E)} \frac{d u d v}{v^{2}}=\iint_{E} \frac{\partial(u, v)}{\partial(x, y)} \frac{d x d y}{v^{2}}=\iint_{E} \frac{1}{|c z+d|^{4}} \frac{|c z+d|^{4}}{y^{2}} d x d y=\mu(E) .
$$

Um polígono hiperbólico de $n$ lados (ou simplesmente polígono) é um conjunto fechado na fechadura de $\mathbb{H}$ em $\mathbb{C}$ limitado por $n$ segmentos de $\mathbb{H}$-linhas (chamados de lados ou arestas). A interseção de dois de tais segmentos é chamado um vértice (permitimos vértices em $\mathbb{R} \cup\{\infty\}$ ). $\mathrm{O}$ ângulo entre duas $\mathbb{H}$-linhas em $\mathbb{H}$ é definido como o ângulo entre suas retas tangentes no seu ponto de interseção e será zero se as $\mathbb{H}$-linhas intersectam-se em um ponto de $\mathbb{R} \cup\{\infty\}$. Como na geometria Euclideana ao ângulo entre dois lados cuja medida é $\pi / 2$ é chamado ângulo reto. Dizemos que um polígono é reto se todos seus ângulos são retos.

Observação 1.45. Qualquer elemento de $\operatorname{Isom}^{+}(\mathbb{H})=P S L(2, \mathbb{R})$ é conforme, isto é, preserva ângulos. Isto segue-se do fato conhecido: se $f: A \subset \mathbb{C} \rightarrow \mathbb{C}$ é holomorfa em $A$ com $f^{\prime}(z) \neq 0$ para todo $z \in A$, então $f$ é conforme.

Teorema 1.46 (Gauss-Bonnet). Seja $\Delta$ um triângulo hiperbólico com ângulos $\alpha, \beta, \gamma$. Então $\mu(\Delta)=\pi-\alpha-\beta-\gamma$.

Prova: Temos três casos:

Caso 1. Consideramos o caso onde dois lados de $\Delta$ são $\mathbb{H}$-linhas verticais. Então, a base de $\Delta$ é um segmento de um semi-círculo Euclideano. Aplicando as transformações da forma $z \mapsto z+k(k \in \mathbb{R}) z \mapsto \lambda z(\lambda>0)$ podemos assumir que o semi-círculo tem 
centro 0 e raio 1, estas transformações não mudam a H-área pelo Teorema 1.44 , e os ângulos também são preservados pela conformalidade das tranformações. Ver Figura 1.2. Os ângulos $A O C$ e $B O D$ são iguais a $\alpha$ e $\beta$, respectivamente. Assumamos que as

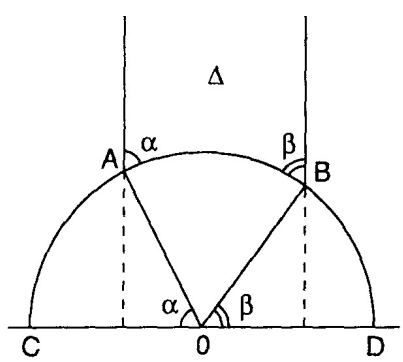

Figura 1.2: Triângulo hierbólico $\Delta$ com dois lados verticais

$\mathbb{H}$-linhas através de $A$ e $B$ são $x=a$ e $x=b$ respectivamente. Agora calculamos

$$
\mu(\Delta)=\iint_{\Delta} \frac{d x d y}{y^{2}}=\int_{a}^{b} d x \int_{\sqrt{1-x^{2}}}^{\infty} \frac{d y}{y^{2}}=\int_{a}^{b} \frac{d x}{\sqrt{1-x^{2}}}
$$

Fazemos a substituição $x=\cos \theta(0 \leq \theta \leq \pi)$, então

$$
\mu(\Delta)=\int_{\pi-\alpha}^{\beta} \frac{-\sin \theta}{\sin \theta} d \theta=\pi-\alpha-\beta
$$

Caso 2. Assumamos que $\Delta$ tem um vértice na reta real. Aplicando uma transformção de $\operatorname{PSL}(2, \mathbb{R})$ podemos mapear este vértice no $\infty$ sem mudar a $\mathbb{H}$-área e os ângulos. Logo, se os ângulos não zeros de $\Delta$ são $\alpha$ e $\beta$ então $\mu(\Delta)=\pi-\alpha-\beta$ pelo Caso 1.

Caso 3. $\Delta$ não tem vértices em $\mathbb{R} \cup\{\infty\}$. Suponhamos que $\Delta$ tem vértices $A, B, C$ e que a prolongação do segmento $A B$ intersecta a $\mathbb{R}$ in $D$ e podemos supor que nenhum de seus lados é uma $\mathbb{H}$-linha vertical. Assim temos uma situação como na Figura 1.3 . Aqui, $\Delta=\Delta_{1}-\Delta_{2}$ onde $\Delta_{1}$ tem vértices $A, C, D$ e $\Delta_{2}$ tem vértices $B, C, D$.

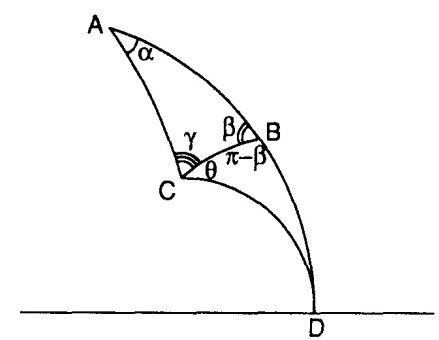

Figura 1.3:

Então $\mu(\Delta)=\mu\left(\Delta_{1}\right)-\mu\left(\Delta_{2}\right)=\pi-\alpha-(\gamma+\theta)-[\pi-\theta-(\pi-\beta)]=\pi-\alpha-\beta-\gamma$. 
Corolário 1.47. Seja П um polígono hiperbólico estrelado ${ }^{1}$ de $n$ lados com ângulos $\alpha_{1}, \ldots, \alpha_{n}$. Então

$$
\mu(\Pi)=(n-2) \pi-\alpha_{1}-\ldots-\alpha_{n}
$$

Prova: Sejam $A_{1}, \ldots, A_{n}$ os vértices de $\Pi$ e seja $O$ o ponto no interior de $\Pi$ tal que os segementos $O A_{1}, \ldots, O A_{n}$ estão em $\Pi$. O resultado segue-se de somar as $\mathbb{H}$-áreas dos triângulos $O A_{1} A_{2}, O A_{2} A_{3}, \ldots, O A_{n} A_{1}$.

Lema 1.48. Ver [8, pág.236].

Sejam $\alpha, \beta, \gamma$ números reais não negativos tal que $\alpha+\beta+\gamma<\pi$. Então existe um triângulo hiperbólico com ângulos $\alpha, \beta, \gamma$.

Lema 1.49. Dado três números positivos $a, b, c$, existe um hexágono hiperbólico reto con lados disjuntos não adjacentes de comprimento hiperbólico $a, b, c$.

Prova: Utilizamos o modelo $\mathbb{H}$. Consideremos as geodésicas perpendiculares $\beta, a$ e $\gamma$ em $\mathbb{H}$ como na Figura 1.4 . Seja $B$ o conjunto de todos os pontos em $\mathbb{H}$ que estam a uma distância $c$ de $\beta$ e no mesmo lado de $\gamma($ Em $\mathbb{H}, B$ é a linha reta Euclideana que passa pela origem). Agora consideremos uma geodésica $\alpha$ tangente a $B$ e movemos $\alpha$ ao longo de $B$ até que $\rho(\gamma, \alpha)=b$. Portanto temos o hexágono requerido.

Nesta ultima parte vamos apresentar algumas fórmulas de trigonometria hiperbólica,

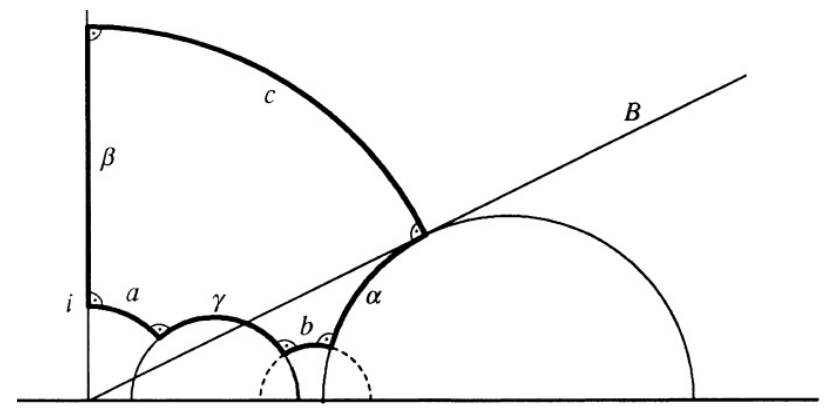

Figura 1.4: Construção de um hexágono hiperbólico reto

as quais utilizaremos mais na frente, cujas provas podem-se encontrar em [1, Capítulo 7] ou [3, Capítulo 2].

Proposição 1.50. Para um quadrilátero hiperbólico com três ângulos retos (triretângulo) com lados rotulados como na Figura 1.5 temos:

(i) $\cos \varphi=\sinh a \sinh b$.

(ii) $\cosh a=\cosh \alpha \sin \varphi$.

\footnotetext{
${ }^{1}$ Um subconjunto $C$ de $\mathbb{H}$ é estrelado se existe um ponto $O$ no interior de $C$ tal que para qualquer ponto $P$ de $C$ existe um segmento de $\mathbb{H}$-linha contido em $C$ juntando $O$ e $P$.
} 


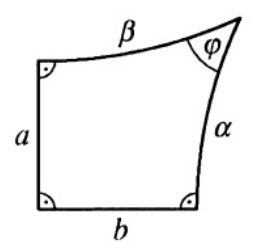

Figura 1.5: Triretângulo

Proposição 1.51 (Pentágonos retos). Para qualquer pentágono reto com lados consecutivos $a, b, \alpha, c, \beta$ temos:

(i) $\cosh c=\sinh a \sinh b$,

(ii) $\cosh =\operatorname{coth} \alpha \operatorname{coth} \beta$.

Proposição 1.52 (Hexágonos retos). Para qualquer hexágono reto convexo com lados consecutivos $a, \gamma, b, \alpha, c, \beta$ se cumpre:

(i) $\cosh c=\sinh a \sinh b \cosh \gamma-\cosh a \cosh b$,

(ii) $\sinh a: \sinh \alpha=\sinh b: \sinh \beta=\sinh c: \sinh \gamma$,

(iii) $\operatorname{coth} \alpha \sinh \gamma=\cosh \gamma \cosh b-\operatorname{coth} a \sinh b$.

Proposição 1.53. Para qualquer hexágono reto com lados $a, \gamma, b, \alpha, c, \beta$ com lados $c$ e $\gamma$ intersectandose (Ver Figura 1.6), temos $\cosh c=\sinh a \sinh b \cosh \gamma+\cosh a \cosh b$.

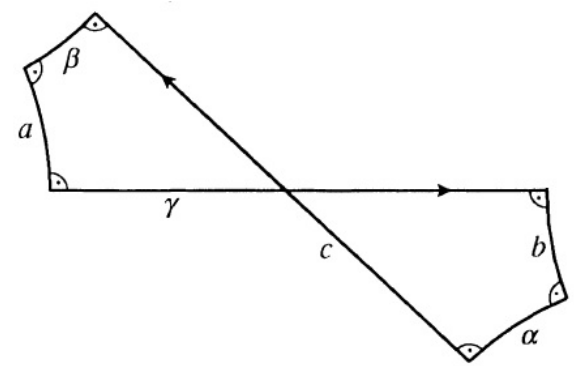

Figura 1.6: Hexágono hiperbólico reto com autointerseção

\subsection{Grupos Fuchsianos}

Nesta seção, vamos definir os grupos Fuchsianos e veremos algumas de suas propriedades as quais serão utilizados nos próximos capítulos. Uma excelente referência é [10].

Para começar, fixaremos uma topologia em $\operatorname{PSL}(2, \mathbb{R})$ dada na seguinte observação.

Observação 1.54. $P S L(2, \mathbb{R})$ é um grupo topológico ${ }^{2}$, cuja topologia é a topologia quociente sob a aplicação $M$ (Ver Observação 1.25). Como $S L(2, \mathbb{R})$ pode ser identificado

\footnotetext{
${ }^{2}$ Um conjunto $G$ é um grupo topológico, se $G$ possui uma estrtura de grupo $(G, \cdot)$ e uma topologia $\Omega$ que tal que as funções $(a, b) \mapsto a \cdot b$ e $a \mapsto a^{-1}$ são funções contínuas.
} 
com um subespaço de $\mathbb{R}^{4}$, a saber, $\left\{(a, b, c, d) \in \mathbb{R}^{4} \mid a d-b c=1\right\}$. A topologia de $S L(2, \mathbb{R})$ é a induzida pela topologia Euclideana de $\mathbb{R}^{4}$, isto é, a sequência $\left\{A_{n}\right\}_{n=1}^{\infty}$ de $S L(2, \mathbb{R})$ com

$$
A_{n}=\left[\begin{array}{ll}
a_{n} & b_{n} \\
c_{n} & d_{n}
\end{array}\right]
$$

converge para

$$
A=\left[\begin{array}{ll}
a & b \\
c & d
\end{array}\right]
$$

em $S L(2, \mathbb{R})$ se e somente se $a_{n}, b_{n}, c_{n}$ e $d_{n}$ converge para $a, b, c$ e $d$, respectivamente.

Os pontos fixos das transformações de $P S L(2, \mathbb{R})$ jogam um papel importante. Os pontos fixos são encontrados resolvendo a equação

$$
z=\frac{a z+b}{c z+d}, \quad a, b, c, d \in \mathbb{R}, \quad a d-b c=1
$$

e como $a, b, c, d$ são reais vemos que esta transformação tem ou dois pontos fixos em $\mathbb{R} \cup\{\infty\}$, um ponto fixo em $\mathbb{R} \cup\{\infty\}$, ou um par de pontos fixos complexos conjugados. Devido a isto, classificamos os elementos de $P S L(2, \mathbb{R})$ na seguinte definição.

Definição 1.55. Seja $T(z)=\frac{a z+b}{c z+d} \in P S L(2, \mathbb{R})$, dizemos que:

(i) T é um elemento parabólico se satifaz $|a+d|=2$.

(ii) T é um elemento hiperbólico se satisfaz $|a+d|>2$.

(iii) T é um elemento elíptico se satisfaz $|a+d|<2$.

Observação 1.56. 1.- Seja $T$ um elemento parabólico, então $T$ tem um único ponto fixo $\alpha \in \mathbb{R} \cup\{\infty\}$. Pelo Teorema 1.28 (ii) existe $S \in P S L(2, \mathbb{R})$ tal que $S(\alpha)=\infty$. Daqui $S T S^{-1}$ é parabólico com ponto fixo $\infty$ e portanto

$$
W=S T S^{-1}: z \mapsto z+t, \quad t \in \mathbb{R} \backslash\{0\}
$$

Seja $V(z)=(1 /|t|) z$. Então $V W V^{-1}: z \mapsto z \pm 1$, o sinal depende em se $t>0$ ou $t<0$. Pode-se mostrar que $z \mapsto z+1$ não é conjugado a $z \mapsto z-1$ em $P S L(2, \mathbb{R})$. Portanto, existem duas classes de conjugação de elementos parabólicos em $P S L(2, \mathbb{R})$.

2.- Seja $T$ o elemento hiperbólico, então $T$ tem dois pontos fixos $\alpha, \beta \in \mathbb{R} \cup\{\infty\}$. Pelo Teorema $1.28($ ii $)$, existe $S \in P S L(2, \mathbb{R})$ tal que $S$ leva $\alpha$ a 0 e $\beta$ a $\infty$. Assim

$$
S T S^{-1}=U_{\lambda}, \quad \lambda \in \mathbb{R} \backslash\{0,1\} .
$$

onde $U_{\lambda}=\lambda z$. Se $B(z)=-1 / z$ então $B U_{\lambda} B^{-1}=U_{\lambda^{-1}}$, assim $U_{\lambda}$ é conjugado a $U_{\lambda^{-1}}$ 
em $\operatorname{PSL}(2, \mathbb{R})$, mas $U_{\lambda}$ não é conjugado a $U_{\kappa}$ se $k \neq \lambda$ ou $\lambda^{-1}$. Portanto, todo elemento hiperbólico de $P S L(2, \mathbb{R})$ é conjugado a um único elemento da forma $U_{\lambda}, \lambda>1$.

3.- Seja T um elemento elíptico, então $T$ tem um ponto fixo $\xi \in \mathbb{H}$ e outro conjugado. Pelo Teorema $1.28(i)$ existe $S \in P S L(2, \mathbb{R})$ tal que $S(\xi)=i$. Então $S(\bar{\xi})=-i$ e $W=$ $S T S^{-1}$ é um elemento elíptico com pontos fixos $i$ e $-i$. Portanto

$$
\frac{W(z)-i}{W(z)+i}=\lambda\left(\frac{z-i}{z+i}\right)
$$

Como $W$ mapea $\mathbb{R} \cup\{\infty\}$ sobre se mesmo, podemos encontrar $\alpha \in \mathbb{R}$ tal que $W(\alpha) \in \mathbb{R}$. Daqui

$$
\left|\frac{W(\alpha)-i}{W(\alpha)+i}\right|=\left|\frac{\alpha-i}{\alpha+i}\right|=1
$$

e assim $\lambda=e^{i \theta}(0 \leq \theta<2 \pi)$. Portanto $T$ é conjugado a $W$, onde

$$
\frac{W(z)-i}{W(z)+i}=e^{i \theta}\left(\frac{z-i}{z+i}\right), \quad(0 \leq \theta<2 \pi)
$$

Não podemos obter uma forma mais simples em $\operatorname{PSL}(2, \mathbb{R})$, mas é importante notar que se

$$
\frac{z-i}{z+i}=z^{\prime}, \frac{W(z)-i}{W(z)+i}=w^{\prime}
$$

então $z^{\prime}, w^{\prime} \in \Delta$ e $w^{\prime}=e^{i \theta} z^{\prime}$. Portanto, em $P S L(2, \mathbb{C}), T$ é conjugado a uma rotação do disco unitário.

Definição 1.57. Um subgrupo discreto ${ }^{3} \Gamma$ de $P S L(2, \mathbb{R})$ é chamado um grupo Fuchsiano.

Lema 1.58. Para um subgrupo $\Gamma$ de $P S L(2, \mathbb{R})$, são equivalentes:

(i) Гé um grupo Fuchsiano.

(ii) Não existe uma sequência de elementos distintos em $\Gamma$ a qual converge em $P S L(2, \mathbb{R})$.

Prova: $\quad(i) \Rightarrow(i i)$. Suponhamos que existe uma sequência $\left\{\gamma_{n}\right\}_{n=1}^{\infty}$ de elementos distintos de $\Gamma$ a qual converge a $\gamma \in P S L(2, \mathbb{R})$, então $\left\{\gamma_{n}^{-1}\right\}_{n=1}^{\infty}$ converge para $\gamma^{-1}$ em $P S L(2, \mathbb{R})$. Temos que $\gamma_{n}^{-1} \circ \gamma_{n+1} \in \Gamma$ e $\gamma_{n}^{-1} \circ \gamma_{n+1} \neq I d$ para qualquer $n$, o que implica que o elemento $I d$ não é um ponto isolado de $\Gamma$, e assim $\Gamma$ não é discreto. $(i i) \Rightarrow(i)$. Segue-se da definição de grupo Fuchsiano.

\footnotetext{
${ }^{3}$ Um subconjunto $A$ de um espaço topológico é discreto, se para todo ponto $p$ de $A$, existe um aberto $U_{p}$ de $X$ tal que $U_{p} \cap X=\{p\}$.
} 
Definição 1.59. Dado $\gamma \in P S L(2, \mathbb{R})$ um elemento hiperbólico, com seus pontos fixos $a, b \in$ $\mathbb{R}$, a geodésica juntando a e b, denotado por $E_{\gamma}$, é chamado o eixo de $\gamma$. O ponto a é chamado atrator se para todo $z \in \mathbb{H}$ temos que $\gamma^{n}(z) \rightarrow$ a quando $n \rightarrow+\infty$ e o ponto b é chamado repulsor se para todo $z \in \mathbb{H}$ temos que $\gamma^{-n}(z) \rightarrow$ b quando $n \rightarrow+\infty$.

Como qualquer elemento $T \in P S L(2, \mathbb{R})$ leva geodésicas em geodésicas, então $\gamma$ mapea $E_{\gamma}$ sobre $E_{\gamma}$.

Exemplo 1.60. Seja $\gamma(z)=\lambda z, \lambda>1$, então $\gamma$ é hiperbólico com pontos fixos 0 e $\infty$. Neste caso, $E_{\gamma}$ é a parte positiva do eixo imaginário, $\infty$ é o ponto atrator de $\gamma$ e 0 é o ponto repulsor de $\gamma$.

Se $\gamma \in P S L(2, \mathbb{R})$, denotamos por $F i x(\gamma)$ o conjuntos de seus pontos fixos.

Lema 1.61. Sejam $\gamma$ e $\delta$ dois elementos de um grupo Fuchsiano $\Gamma$. Se $\gamma$ é hiperbólico e $\delta \neq I d$, então cumpre-se um dos seguintes:

(i) $\operatorname{Fix}(\gamma)=\operatorname{Fix}(\delta)$

(ii) $\operatorname{Fix}(\gamma) \cap F i x(\delta)=\emptyset$

Prova: Suponhamos que não se cumpra $(i)$ nem $(i i)$. Por $P S L(2, \mathbb{R})$-conjugação, podemos supor que $\operatorname{Fix}(\gamma)=\{0, \infty\}$ e $F i x(\gamma) \cap F i x(\delta)=\{\infty\}$. Então as representações matriciais $A, B$ de $\gamma, \delta$ são dados por

$$
\begin{gathered}
A=\left[\begin{array}{cc}
\lambda & 0 \\
0 & \lambda^{-1}
\end{array}\right], \quad \lambda>0, \lambda \neq 1 \\
B=\left[\begin{array}{cc}
a & b \\
0 & a^{-1}
\end{array}\right], \quad a, b \in \mathbb{R}, \quad a b \neq 0 .
\end{gathered}
$$

respectivamente. Agora, pondo $C_{n}=B A^{n} B^{-1} A^{-n}$ para qualquer positivo $n$, obtemos

$$
C_{n}=\left[\begin{array}{cc}
1 & a b\left(1-\lambda^{2 n}\right) \\
0 & 1
\end{array}\right]
$$

Daqui, se $0<\lambda<1$, então $C_{n}$ converge para

$$
C=\left[\begin{array}{cc}
1 & a b \\
0 & 1
\end{array}\right]
$$

quando $n \rightarrow+\infty$. Se $\lambda>1$, então $C_{n} \rightarrow C$ quando $n \rightarrow-\infty$. Desde que $a b \neq 0, \lambda>0$, e $\lambda \neq 1$, vemos que a sequência $\left\{C_{n}\right\}_{n=-\infty}^{\infty}$ consiste de elementos distintos. Portanto, pelo Lema 1.58, teríamos que $\Gamma$ não é Fuchsiano, o qual é uma contradição. 
Lema 1.62. Seja $\Gamma$ um grupo Fuchsiano contendo a traslação $\gamma_{0}(z)=z+1$. Então todo elemento $\gamma$ tendo representação matricial

$$
A=\left[\begin{array}{ll}
a & b \\
c & d
\end{array}\right], \quad a, b, c, d \in \mathbb{R}, a d-b c=1
$$

satisfaz $|c| \geq 1$ sempre que $c \neq 0$.

Prova: Suponhamos que exista um elemento $\gamma \in \Gamma$ com representação matricial $A \in$ $S L(2, \mathbb{R})$ tal que $0<|c|<1$. Temos que

$$
A_{0}=\left[\begin{array}{ll}
1 & 1 \\
0 & 1
\end{array}\right]
$$

é a representação matricial de $\gamma_{0}$. Pondo, $A_{1}=A$ e $A_{n+1}=A_{n} A_{0} A_{n}^{-1}$ para qualquer inteiro positivo $n$, vemos que $A_{n}$ escreve-se na forma

$$
A_{n}=\left[\begin{array}{ll}
a_{n} & b_{n} \\
c_{n} & d_{n}
\end{array}\right]
$$

onde $a_{n}=1-a_{n-1} c_{n-1}, b_{n}=a_{n-1}^{2}, c_{n}=-c_{n-1}^{2}$ e $d_{n}=1+a_{n-1} c_{n-1}$. Assim, temos que $c_{n}=-c^{2^{n-1}} \rightarrow 0$ quando $n \rightarrow+\infty$. Pondo $M=\max \{|a|, 1 /(1-|c|)\}$, obtemos por indução que $\left|a_{n}\right| \leq M$ para qualquer $n$. Logo, cada $a_{n}, b_{n}$ e $d_{n}$ converge para 1 quando $n \rightarrow+\infty$. Portanto $A_{n}$ converge para $A_{0}$, o que contradiz a discretitude de $\Gamma$.

Definição 1.63. Uma família $\left\{M_{\alpha} \mid \alpha \in \Lambda\right\}$ de conjuntos de um espaço topológico $X$ é chamado localmente finito se para qualquer subconjunto compacto $K \subset X$ tem-se $M_{\alpha} \cap K \neq$ $\emptyset$ apenas para un número finito de indices $\alpha \in \Lambda$.

Definição 1.64. Seja G um grupo de homeomorfismos de um espaço topológico X. Dizemos que $G$ age propriamente descontínua em $X$ se cada ponto $x \in X$ tem uma vizinhança $V$ tal que se $g(V) \cap V \neq \emptyset$ para $g \in G$, então $g(x)=x$.

Proposição 1.65. Seja $X$ um espaço métrico localmente compacto e G um grupo de homeomorfismos de $X$. Se para qualquer subconjunto compacto $K \in X$, tem-se que $g(K) \cap K \neq \emptyset$ apenas para um número finito de elementos $g \in G$ então $G$ age propriamente descontínua em $X$.

Prova: Seja $x \in X$, como $X$ é localmente compacto, existe um aberto $W \operatorname{com} x \in W$ tal que $\bar{W}$ é compacto. Seja $\left\{\gamma_{0}=I d, \gamma_{1}, \ldots, \gamma_{n}, \gamma_{n+1}, \ldots, \gamma_{n+m}\right\}=\{g \in G \mid g(\bar{W}) \cap \bar{W} \neq \emptyset\}$ 
com $\gamma_{i}(x)=x, i=1, \ldots, n$ e consideremos $V=W \backslash \bigcup_{j=1}^{m} \gamma_{n+j}(\bar{W})$ então $V$ é aberto com $x \in V$ e se $g(V) \cap V \neq \emptyset$ por construção temos que $g(x)=x$.

Lema 1.66. Seja $z \in \mathbb{H}$ e seja $K$ um subconjunto compacto de $\mathbb{H}$. Então o conjunto

$$
E=\{T \in P S L(2, \mathbb{R}) \mid T(z) \in K\}
$$

é compacto.

Prova: Lembrar que $P S L(2, \mathbb{R})$ tem a topologia quociente induzida por $S L(2, \mathbb{R})$. Assim, temos a aplicação quociente $q: S L(2, \mathbb{R}) \rightarrow P S L(2, \mathbb{R})$ definido por

$$
q\left(\begin{array}{ll}
a & b \\
c & d
\end{array}\right)=T, \quad \text { onde } T(w)=\frac{a w+b}{c w+d}
$$

Se mostrarmos que

$$
E_{1}=\left\{\left(\begin{array}{ll}
a & b \\
c & d
\end{array}\right) \in S L(2, \mathbb{R}) \mid \frac{a z+b}{c z+d} \in K\right\}
$$

é compacto, então segue-se que $E=q\left(E_{1}\right)$ é compacto. Vamos provar que $E_{1}$ é compacto mostrando que é fechado e limitado considerado como um subconjunto de $\mathbb{R}^{4}$. Temos a aplicação $\beta: S L(2, \mathbb{R}) \rightarrow H$ definida por $\beta(A)=q(A)(z)$, e como $E_{1}=\beta^{-1}(K)$ segue-se que $E_{1}$ é fechado.

Vejamos que $E_{1}$ é limitado. Como $K$ é limitado existe $M_{1} \in \mathbb{R}$ tal que

$$
\left|\frac{a z+b}{c z+d}\right| \leq M_{1}
$$

para todo $\left(\begin{array}{ll}a & b \\ c & d\end{array}\right) \in E_{1}$. Também existe $M_{2}>0$, pela compacidade de $K$ em $\mathbb{H}$, tal
que que

$$
\frac{\operatorname{Im}(z)}{|c z+d|^{2}}=\operatorname{Im}\left(\frac{a z+b}{c z+d}\right) \geq M_{2}
$$

. Assim, obtemos

$$
|c z+d| \leq \sqrt{\frac{\operatorname{Im}(z)}{M_{2}}} \text { e }|a z+b| \leq M_{1} \sqrt{\frac{\operatorname{Im}(z)}{M_{2}}}
$$

Portanto, temos que $a, b, c, d$ são limitados. 
Corolário 1.67. Seja $z \in \mathbb{H}$ e seja $K$ um subconjunto compacto de $\mathbb{H}$. Se $\Gamma$ é um grupo Fuchsiano, então

$$
\{T \in \Gamma \mid T(z) \in K\}
$$

éfinito.

Teorema 1.68. Seja $\Gamma$ um subgrupo de $\operatorname{PSL}(2, \mathbb{R})$. Então

(i) Гé Fuchsiano se e somente se $\Gamma$ age propriamente descontínua em $\mathbb{H}$.

(ii) Seja $p \in \mathbb{H}$ fixado por algum elemento de $\Gamma$, então existe uma vizinhança $W$ de $p$ tal que nenhum outro ponto de $W$ é fixado por um elemento de $\Gamma-\{I d\}$.

Prova: Vejamos que um grupo Fuchsiano age propriamente descontínua em $\mathbb{H}$. Seja $z_{0} \in \mathbb{H}$ e seja $\overline{B_{\varepsilon}\left(z_{0}\right)}$ o disco hiperbólico fechado de raio $\epsilon$ com centro $z_{0}$, temos que $\overline{B_{\varepsilon}\left(z_{0}\right)}$ é compacto (pois a topologia da métrica hiperbólica coincide com a topologia Euclideana). Pelo Corolário 1.67

$$
\left\{T \in \Gamma \mid T\left(z_{0}\right) \in \overline{B_{\varepsilon}\left(z_{0}\right)}\right\}
$$

é finito. Assim, existe $0<\delta<\varepsilon$ tal que $\overline{B_{\delta}\left(z_{0}\right)}$ não contém outro ponto da $\Gamma$-órbita de $z_{0}$. Ponhamos $V=\overline{B_{\delta / 2}\left(z_{0}\right)}$, se $V \cap S(V) \neq \emptyset$ para $S \in \Gamma$, existe $z \in V$ tal que $S(z) \in \Gamma$. Daqui, $\rho\left(z, z_{0}\right) \leq \delta / 2, \rho\left(S(z), z_{0}\right) \leq \delta / 2$ e assim

$$
\begin{aligned}
\rho\left(z_{0}, S\left(z_{0}\right)\right) & \leq \rho\left(z_{0}, S(z)\right)+\rho\left(S(z), S\left(z_{0}\right)\right) \\
& =\rho\left(z_{0}, S(z)\right)+\rho\left(z, z_{0}\right) \leq \delta
\end{aligned}
$$

e pela definição de $\delta$, temos que $S\left(z_{0}\right)=z_{0}$. Portanto $\Gamma$ age propriamente descontínua em $\mathbb{H}$.

Antes de provar o recíproco de $(i)$, vamos provar $(i i)$. Suponhamos que $p$ é um ponto fixado por $S \neq I d$. Então, pelo que acabamos de provar, existe uma vizinhança $W$ de $p$ tal que $W \cap S(W) \neq \emptyset$ implica que $S(p)=p$. Se $q \in W$ é fixado por $T \neq I d$, então $T(W) \cap W \neq \emptyset$ e daí $T(p)=p$, portanto $p=q$.

Agora provamos o recíproco do item $(i)$, isto é, devemos provar que um subgrupo de $P S L(2, \mathbb{R})$ que age propriamente descontínua em $\mathbb{H}$ deve ser discreto. Suponhamos que isso não acontece, e escolhamos um ponto $s \in \mathbb{H}$ que não é fixado por qualquer elemento de $\Gamma$ diferente da identidade (tal ponto existe pela parte $(i i)$ ). Como estamos supondo que $\Gamma$ não é discreto, existe uma sequência de elementos distintos $\left\{T_{k}\right\}_{k=1}^{\infty}$ tal que $T_{k} \rightarrow I d$ quando $k \rightarrow \infty$. Daqui $T_{k}(s) \rightarrow s$ quando $k \rightarrow \infty$ e como $s$ não é fixado 
por qualquer elemento de $\Gamma$ diferente da identidade, $\left\{T_{k}(s)\right\}_{k=1}^{\infty}$ é uma sequência de pontos distintos. Portanto toda vizinhança de $s$ contém outros pontos da $\Gamma$-órbita de $s, \log$ $\Gamma$ não age propriamente descontínua.

Corolário 1.69. Seja $\Gamma$ um subgrupo de $P S L(2, \mathbb{R})$. Então $\Gamma$ é um grupo Fuchsiano se e somente se para todo $z \in \mathbb{H} a \Gamma$-órbita de z, denotado por $\Gamma z$,é um subconjunto discreto de $\mathbb{H}$.

Prova: Suponhamos que $\Gamma z$ é um subconjunto discreto de $\mathbb{H}$, então existe um $\varepsilon$ tal que o disco hiperbólico $B_{\varepsilon}(z)$ não contém outro ponto de $\Gamma z$.

Daqui, se $V \subset B_{\varepsilon / 2}(z)$, podemos mostrar que $V \cap S(V) \neq \emptyset$ implica que $S(z)=z$ (analogamente como na prova do item $(i)$ do Teorema 1.68 ) e portanto $\Gamma$ age propriamente descontínua em $\mathbb{H}$ e segue-se que $\Gamma$ é um grupo Fuchsiano.

Reciprocamente, se $\Gamma$ é um grupo Fuchsiano então ele age propriamente descontínua em $\mathbb{H}$ e daí toda $\Gamma z$ é discreta em $\mathbb{H}$.

Corolário 1.70. Se Г é um grupo Fuchsiano, então o conjunto dos pontos fixos de elementos elípticos não tem pontos de acumulação em $\mathbb{H}$.

Prova: Seja $z \in \mathbb{H}$ e $K$ um conjunto compacto tal que $z \in K$. Suponhamos que $z=T z$ para algum $T \in \Gamma$. O Teorema 1.68 e a Proposição 1.65 (no caso de $X=\mathbb{H}$ e $G=\Gamma$ a recíproca é certa) implicam que isto só é possível para um número finito de $T \in \Gamma$, daqui só existe um número finito de pontos fixos elípticos em $K$.

Observação 1.71. O Corolário 1.69 implica o seguinte: Se $z \in \mathbb{H}$ e $\left\{T_{n}\right\}_{n=1}^{\infty}$ é uma sequência de elementos distintos de $\Gamma$, então se $\left\{T_{n}(z)\right\}_{n=1}^{\infty}$ tem um ponto límite $\alpha \in$ $\mathbb{C} \cup\{\infty\}$ então $\alpha \in \mathbb{R} \cup\{\infty\}$.

Definição 1.72. O conjunto de todos os possíveis pontos límites das $\Gamma$-órbitas $\Gamma z, z \in \mathbb{H}$,é chamado o conjunto limite de $\Gamma$ e é denotado por $\Lambda(\Gamma)$.

Observação 1.73. Sejam $S, T \in P S L(2, \mathbb{R})$. Se $S T=T S$ então $S$ mapea o conjunto dos pontos fixos de $T$ sobre se mesmo.

Vamos olhar para os centralizadores dos elementos parabólicos, elípticos e hiperbólicos em $P S L(2, \mathbb{R})$. Suponhamos que $T(z)=z+1$. Se $S \in C_{P S L(2, \mathbb{R})}(T)^{4}$ então $S(\infty)=\infty$. Portanto $S(z)=a z+b, S T=T S$ nos dá que $a=1$ e assim $C_{P S L(2, \mathbb{R})}(T)=\{z \mapsto z+k \mid k \in \mathbb{R}\}$. Com o mesmo resultado para o centralizador de $z \mapsto z-1$.

\footnotetext{
${ }^{4}$ Se $G$ é um grupo e $g \in G$, então o centralizador de $g$ em $G$ é dado por $C_{G}(g)=\{h \in G \mid h g=g h\}$
} 
Similarmente pode-se ver que o centralizador de $U_{\lambda}(\lambda>0, \lambda \neq 1)$ em $P S L(2, \mathbb{R})$ coniste de transformações da forma $U_{\mu}(\mu>0)$ e o centralizador do elemento elíptico (1.4) consiste das transformações

$$
\frac{W(z)-i}{W(z)+i}=e^{i \phi}\left(\frac{z-i}{z+i}\right) \quad(0 \leq \phi<2 \pi)
$$

Destas observações se obtém o seguinte resultado.

Teorema 1.74. (i) Dois elementos distintos da identidade em $P S L(2, \mathbb{R})$ commutam se e somente se eles têm o mesmo conjunto de pontos fixos.

(ii) O centralizador em $\operatorname{PSL}(2, \mathbb{R})$ de um elemento hiperbólico (respectivamente, parabólico, elíptico) de $\operatorname{PSL}(2, \mathbb{R})$ consiste de todos os elementos hiperbólicos (respectivamente, parabólicos, elípticos) com o mesmo conjunto de pontos fixos, junto com a identidade.

Lema 1.75. Ver [10, pág.29].

(i) Qualquer subgrupo discreto não trivial de $\mathbb{R}$, o grupo aditivo dos reais, é cíclico infinito.

(ii) Qualquer subgrupo discreto de $S^{1}$, o grupo multiplicativo dos números complexos de módulo 1, é cíclico finito.

Proposição 1.76. Seja $\Gamma$ um grupo Fuchsiano cujos todos os elementos distintos da identidade têm o mesmo conjunto de pontos fixos. Então $\Gamma$ é cíclico.

Prova: Suponhamos que $S \in \Gamma$ é hiperbólico. Então escolhendo um grupo conjugado se fosse necessário, podemos assumir que $S$ fixa 0 e $\infty$. Daqui, todas os elementos de $\Gamma$ são hiperbólicos e fixam 0 e $\infty$, então $\Gamma$ é um subgrupo discreto de $H=\{z \mapsto \lambda z \mid \lambda>$ $0\}$, mas $H$ é isomorfo como grupo topológico a $\mathbb{R}^{*}$ o qual é isomorfo ao grupo aditivo $\mathbb{R}$. Logo, pelo Lema $1.75(i)$, $\Gamma$ é cíclico infinito.

Similarmente, se $\Gamma$ contém um elemento parabólico então $\Gamma$ é um grupo cíclico infinito contendo somente elementos parabólicos. Se $\Gamma$ contém um elemento elíptico então todas as transformações da forma (1.4) formam um grupo isomorfo a $S^{1}$. Lema 1.75 (ii) implica que $\Gamma$ é cíclico finito.

Corolário 1.77. Todo grupo Fuchsiano abeliano é cíclico.

Teorema 1.78. Seja $\Gamma$ um grupo Fuchsiano não cíclico. Então o normalizador de $\Gamma$ em $P S L(2, \mathbb{R})$, $N_{P S L(2, \mathbb{R})}(\Gamma)$, é um grupo Fuchsiano.

Prova: Suponhamos que o normalizador de $\Gamma$ em $P S L(2, \mathbb{R})$ não é Fuchsiano. Então ele contém uma sequência infinita $\left\{T_{n}\right\}_{n=1}^{\infty}$ de elementos distintos tal que $T_{n} \rightarrow I d$ quando $n \rightarrow \infty$. Assim, se $S \in \Gamma(S \neq I d)$, então $T_{n} S T_{n}^{-1} \rightarrow S$ quando $n \rightarrow \infty$. Como 
$\Gamma$ é discreto, existe um interio positivo $m$ tal que $T_{n} S T_{n}^{-1}=S$ para todo $n>m$. Logo, pelo Teorema 1.74, temos que para estos valores de $n, T_{n}$ tem os mesmos pontos fixos de $S$. Mas, como $\Gamma$ não é abeliano, temos que existe $S^{\prime} \in \Gamma$ tal que seu conjunto de pontos fixos não coincide com o de $S$ (Teorema 1.74). Fazendo o mesmo argumento como acima, obtemos que $T_{n}$ tem o mesmo conjunto de pontos fixos que $S^{\prime}$ para $n$ suficientemente grande. Daqui, $S$ e $S^{\prime}$ tem o mesmo conjunto de pontos fixos, a qual é uma contradição. Portanto $N_{P S L(2, \mathbb{R})}(\Gamma)$ é Fuchsiano.

\subsection{Regiões Fundamentais}

A importância de uma região fundamental $F$ de um grupo Fuchsiano $\Gamma$ é que simplesmente nos permite centrarnos em ver como $\Gamma$ age em $F$.

Nesta seção começamos apresentando uma definição um pouco mais geral de região fundamental. Veremos que tais regiões sempre existem para grupos Fuchsianos, como também algumas de suas propiedades junto com um exemplo. No Capítulo 2 também apresentamos outros resultados relacionados a este conceito.

Seja $X$ um espaço métrico e $G$ un grupo de isometrias de $X$ agindo propriamente descontínua em $X$

Definição 1.79. Uma região fechada $F \subset X$ é definida como uma região fundamental para $G$, se:

(i) $\bigcup_{T \in G} T(F)=X$

(ii) $\stackrel{\circ}{F} \cap T(\stackrel{\circ}{F})=\emptyset \quad$ para todo $T \in G-\{I d\}$.

O conjunto $\partial F=F-\stackrel{\circ}{F}$ é chamado a fronteira de $F$. A família $\{T(F) \mid T \in G\}$ é chamado ladrilhamento de $X$.

Teorema 1.80. Sejam $F_{1}$ e $F_{2}$ duas regiões fundamentais para um grupo Fuchsiano $\Gamma$, e $\mu\left(F_{1}\right)<\infty$. Suponhamos que as fronteiras de $F_{1}$ e $F_{2}$ têm área hiperbólica zero. Então $\mu\left(F_{2}\right)=\mu\left(F_{1}\right)$.

Prova: Para $i=1,2$, temos $\mu\left(\stackrel{\circ}{F}_{i}\right)=\mu\left(F_{i}\right)$. Logo

$$
\bigcup_{T \in \Gamma}\left(F_{1} \cap T\left(\stackrel{\circ}{F}_{2}\right)\right)=F_{1} \bigcap\left(\bigcup_{T \in \Gamma} T\left(\stackrel{\circ}{F}_{2}\right)\right) \subseteq F_{1}
$$


Como $\stackrel{\circ}{F}_{2}$ é o interior de uma região fundamental, os conjuntos $F_{1} \cap T\left(\stackrel{\circ}{F}_{2}\right)$ são disjuntos, e assim

$$
\mu\left(F_{1}\right) \geq \sum_{T \in \Gamma} \mu\left(F_{1} \cap T\left(\stackrel{\circ}{F}_{2}\right)\right)=\sum_{T \in \Gamma} \mu\left(T^{-1}\left(F_{1}\right) \cap \stackrel{\circ}{F}_{2}\right)=\sum_{T \in \Gamma} \mu\left(T\left(F_{1}\right) \cap \stackrel{\circ}{F}_{2}\right)
$$

Desde que $F_{1}$ é uma região fundamental, temos

$$
\bigcup_{T \in \Gamma}\left(T\left(F_{1}\right) \cap \stackrel{\circ}{F}_{2}\right)=\stackrel{\circ}{F}_{2}
$$

Daí

$$
\mu\left(F_{2}\right)=\mu\left(\stackrel{\circ}{F}_{2}\right)=\mu\left(\bigcup_{T \in \Gamma} \mu\left(T\left(F_{1}\right) \cap \stackrel{\circ}{F}_{2}\right)\right) \leq \sum_{T \in \Gamma} \mu\left(T\left(F_{1}\right) \cap \stackrel{\circ}{F}_{2}\right)
$$

Portanto $\mu\left(F_{2}\right) \leq \mu\left(F_{1}\right)$. Trocando $F_{1}$ e $F_{2}$, obtemos $\mu\left(F_{1}\right) \leq \mu\left(F_{2}\right)$ e assim $\mu\left(F_{1}\right)=$ $\mu\left(F_{2}\right)$

Definição 1.81. Seja $\Gamma$ um grupo Fuchsiano e seja $p \in H$ um ponto que não é fixado por qualquer elemento de $\Gamma-I d$. O conjunto

$$
D_{p}(\Gamma)=\{z \in H \mid \rho(z, p) \leq \rho(z, T(p)) \text { para todo } T \in \Gamma\}
$$

é chamado a região de Dirichlet para $\Gamma$ centrada em $p$.

Pela invariância da métrica hiperbólica sob $\operatorname{PSL}(2, \mathbb{R})$, esta região pode ser definida como

$$
D_{p}(\Gamma)=\{z \in H \mid \rho(z, p) \leq \rho(T(z), p) \text { para todo } T \in \Gamma\}
$$

Definição 1.82. A mediatriz do segmento geodésico $\left[z_{1}, z_{2}\right]$ é a única geodésica ortogonal a $\left[z_{1}, z_{2}\right]$ passando por $w$, o ponto médio de $\left[z_{1}, z_{2}\right]$.

Lema 1.83. A mediatriz do segmento geodésico $\left[z_{1}, z_{2}\right]$ é dado pela equação

$$
\rho\left(z, z_{1}\right)=\rho\left(z, z_{2}\right)
$$

Prova: Sem perda de generalidade podemos assumir que $z_{1}=i, z_{2}=i r^{2} \operatorname{com} r>0$, assim $w=i r$ e a mediatriz é dada pela equação $|z|=r$. Por outro lado, a equação do lema é equivalente a

$$
\frac{\left|z-z_{1}\right|^{2}}{y}=\frac{\left|z-z_{2}\right|^{2}}{r^{2} y}
$$

e simplifica-se a $|z|=r$ 
Observação 1.84. Seja $T_{1} \in \Gamma$ qualquer, denotamos por $L_{p}\left(T_{1}\right)$ a mediatriz do segmento $\left[p, T_{1}(p)\right]$ e por $H_{p}\left(T_{1}\right)$ o semi-plano contendo $p$ determinado por $L_{p}\left(T_{1}\right)$, então temos

$$
D_{p}(\Gamma)=\bigcap_{T \in \Gamma, T \neq I d} H_{p}(T)
$$

e assim $D_{p}(\Gamma)$ é um fechado hiperbolicamente convexo ${ }^{5}$.

Teorema 1.85. $D_{p}(\Gamma)$ é uma região fundamental conexa para $\Gamma$.

Prova: Seja $z \in H$ e $\Gamma z$ sua $\Gamma$-órbita. Como $\Gamma z$ é discreto, existe $z_{0} \in \Gamma z$ tal que $\rho\left(z_{0}, p\right)$ atinge seu valor mínimo. Assim $\rho\left(z_{0}, p\right) \leq \rho\left(T\left(z_{0}\right), p\right)$ para todo $T \in \Gamma, \log z_{o} \in D_{p}(\Gamma)$. Portanto $D_{p}(\Gamma)$ contém pelo menos um ponto de toda $\Gamma$-órbita.

Se $z$ esta no interior de $D_{p}(\Gamma)$ então $\rho(z, p)<\rho(T(z), p)$ para todo $T \in \Gamma-\{I d\}$. Com efeito, $\rho(p, z)=\rho(T(z), p)$ para algum $T \in \Gamma-\{I d\}$ implica que $\rho(z, p)=\rho\left(z, T^{-1}(p)\right)$ e assim $z \in L_{p}\left(T^{-1}\right)$, então ou $z \notin D_{p}(\Gamma)$ ou $z$ esta na fronteira de $D_{p}(\Gamma)$. Agora, se $z_{1}, z_{2}$ estam no interior de $D_{p}(\Gamma)$, eles não podem estar na mesma $\Gamma$-órbita, caso contrário teríamos $\rho\left(z_{1}, p\right)<\rho\left(z_{2}, p\right)$ e $\rho\left(z_{2}, p\right)<\rho\left(z_{1}, p\right)$, uma contradição. Como $D_{p}(\Gamma)$ é a interseção de semi-planos fechados, temos que $D_{p}(\Gamma)$ é fechado e convexo. Portanto $D_{p}(\Gamma)$ é conexo por caminhos e consequentemente conexo.

Observação 1.86. 1.- Se $F$ é uma região de Dirichlet para $\Gamma$ centrado em $p$, então $T(F)$ é uma região de Dirichlet para $T \Gamma T^{-1}$ centrada em $T(p)$ para qualquer $T \in P S L(2, \mathbb{R})$. 2.- As regiões de Dirichlet são limitadas por $\mathbb{H}-$-linhas e possívelmente por segmentos do eixo real. Se duas $\mathbb{H}$-linhas intersectam-se em $\mathbb{H}$ em um ponto, tal ponto é chamado vértice da região de Dirichlet.

Exemplo 1.87. O grupo modular $\Gamma=P S L(2, \mathbb{Z})$. Pode-se ver que $k i(k>1)$ não é fixado por qualquer elemento diferente da identidade do grupo modular. Fixando $p=k i, k>1$, provaremos que a região

$$
F=\left\{z \in \mathbb{H}|| z|\geq 1,| \operatorname{Re}(z) \mid \leq \frac{1}{2}\right\}
$$

é uma região de Dirichlet para o grupo $P S L(2, \mathbb{Z})$. Temos que as isometrias $T z=z+1$ e $S z=-1 / z$ pertencem a $\Gamma$ e que as três geodésicas de $F$ são $L_{p}(T), L_{p}\left(T^{-1}\right)$ e $L_{p}(S)$. Isto mostra que $D_{p}(\Gamma) \subset F$. Suponhamos $D_{p}(\Gamma) \neq F$, então existem $z \in \stackrel{\circ}{F}$ e $h \in \Gamma$ tal que $h(z) \in \stackrel{\circ}{F}$.

\footnotetext{
${ }^{5}$ Um subconjunto $A$ de $\mathbb{H}$ é hiperbolicamente convexo se dado dois pontos $z, w \in A$ existe um segmento $L$ de uma $\mathbb{H}$-linha juntando os pontos $z$ e $w$ tal que $L \subset A$.
} 


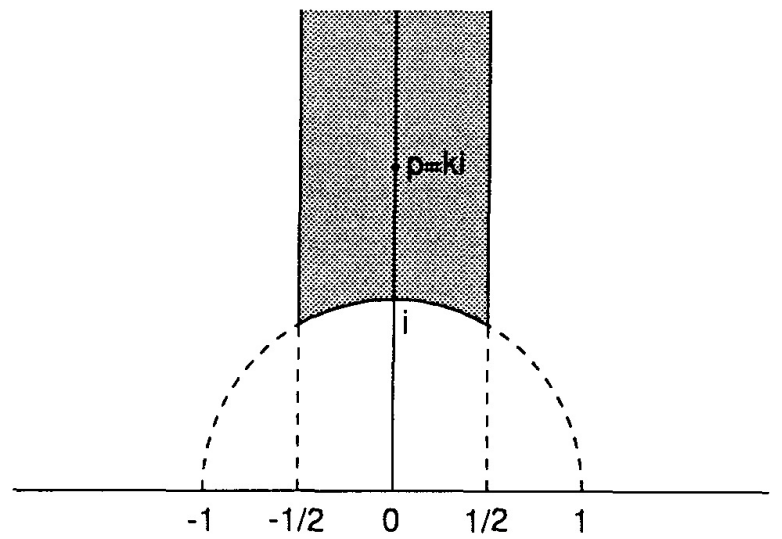

Figura 1.7: Região de Dirichlet de $\operatorname{PSL}(2, \mathbb{Z})$.

Seja

$$
h(z)=\frac{a z+b}{c z+d} \quad(a, b, c, d \in \mathbb{Z}, a d-b c=1)
$$

Então

$$
|c z+d|^{2}=c^{2}|z|^{2}+2 \operatorname{Re}(z) c d+d^{2}>c^{2}+d^{2}-|c d|=(|c|-|d|)^{2}+|c d|
$$

pois $|z|>1$ e $\operatorname{Re}(z)>-\frac{1}{2}$. Este límite inferior é um número inteiro positivo (se fosse igual a 0 , teríamos que $c=d=0$ o que contradiz $a d-b c=1)$. Portanto, pelo menos é 1 , assim $|c z+d|>1$.

Asssim, temos

$$
\operatorname{Imh}(z)=\frac{\operatorname{Im}(z)}{|c z+d|^{2}}<\operatorname{Im}(z)
$$

O mesmo argumento se cumpre com $z$ e $h$ substituído por $h(z)$ e $h^{-1}$, respectivamente, obtendo uma contradição. Portanto $D_{p}(\Gamma)=F$.

Definição 1.88. Dizemos que uma região fundamental $F$ para um grupo Fuchsiano $\Gamma$ é chamada localmente finita se a família $\{T(F) \mid T \in \Gamma\}$ é localmente finita.

Teorema 1.89. Qualquer região de Dirichlet é localmente finita.

Prova: Seja $F=D_{p}(\Gamma)$, onde $p$ não é fixado por qualquer elemento de $\Gamma-\{I d\}$. Seja $a \in F$ e $K \subset \mathbb{H}$ compacto com $a \in K$. Suponhamos que $K \cap T_{i}(F) \neq \emptyset$ para uma sequência infinita $\left\{T_{i}\right\}_{i=1}^{\infty}$ de elementos distintos de $\Gamma$. Seja $\sigma=\sup _{z \in K} \rho(p, z)$. Então $\sigma \leq \rho(p, a)+\rho(a, z)$ para todo $z \in K$, como $K$ é limitado, $\sigma$ é finito. Seja $w_{j} \in K \cap T_{j}(F)$. Então $w_{j}=T_{j}\left(z_{j}\right)$ para $z_{j} \in F$ e daqui

$$
\rho\left(p, T_{j}(p)\right) \leq \rho\left(p, w_{j}\right)+\rho\left(w_{j}, T_{j}(p)\right)=\rho\left(p, w_{j}\right)+\rho\left(z_{j}, p\right) \leq \rho\left(p, w_{j}\right)+\rho\left(w_{j}, p\right) \leq 2 \sigma .
$$


Assim o conjunto infinito de pontos $\left\{T_{i}(p)\right\}_{i=1}^{\infty}$ pertencem à $\mathbb{H}$-bola com centro $p$ e raio $2 \sigma$. Mas isto contradiz o Corolário 1.67.

Seja $F$ uma região fundamental para um grupo Fuchsiano $\Gamma$ e sejam $u$ e $v$ vértices de $F$. Dizemos que $u$ e $v$ são congruentes se existe $T \in \Gamma$ tal que $T(u)=v$. É claro que a congruência é uma relação de equivalência nos vértices de $F$ e as classes de equivalência são chamados ciclos.

Se $u$ é fixado por um elemento elíptico $S$ então $v$ é fixado pelo elemento elíptico $T S T^{-1}$, isto é, se um vértice de um ciclo é fixado por um elemento elíptico também são fixados todos os demais vértices do mesmo ciclo. Tal ciclo é chamado um ciclo elíptico e os vértices desse ciclo são chamados vértices elípticos.

Notar que, se um ponto $w \in \mathbb{H}$ é fixado por algum elemento $S^{\prime} \in \Gamma$, então $w$ pertence à fronteira de $T(F)$ para algum $T \in \Gamma$. Daqui, $u=T^{-1}(w)$ esta na fronteira de $F$ e é fixado pelo elemento elíptico $S=T^{-1} S^{\prime} T$, seja $k$ a ordem de $S$. Suponhamos que $k \geq 3$; então como $S$ é uma isometria hiperbólica fixando $u$, a qual mapea $\mathbb{H}$-linhas a $\mathbb{H}$-linhas, $u$ deve ser um vértice de $F$ cujo ângulo é ao máximo $2 \pi / k$. Se $S$ tem ordem 2 , então seu ponto fixo esta no interior de um lado de $F$. Neste caso $S$ troca os dois segmentos deste lado separados pelo ponto fixo. Vamos incluir tais pontos fixos elípticos como vértices de $F$, o ângulo em tais vértices é $\pi$. Portanto um vértice de $F$ é a interseção em $\mathbb{H}$ de duas $\mathbb{H}$-linhas de $F$ ou o ponto fixo de um elemento elíptico de ordem 2.

Teorema 1.90. Existe uma bijeção entre os ciclos elípticos de $F$ e as classes de conjugação de subgrupos cíclicos finitos maximais não triviais de $\Gamma$.

Prova: Da Proposição 1.76 temos que os subgrupos finitos cíclicos de $P S L(2, \mathbb{R})$ são gerados por elementos elípticos e cada elemento elíptico de $P S L(2, \mathbb{R})$ tem um único ponto fixo em $\mathbb{H}$ e a mesma observação acontece para $\Gamma$. Também, se um ponto em $\mathbb{H}$ tem um estabilizador não trivial em $\Gamma$ então seu estabilizador é um subgrupo finito cíclico de $\Gamma$ (Proposição 1.76) e pela Observação 1.73 este subgrupo finito cíclico é um subgrupo cíclico finito maximal.

Exemplo 1.91. Seja $\Gamma$ o grupo modular. A região de Dirichlet $F$ de $\Gamma$ tem vértices em $\mathbb{H}$ em $z=(-1+i \sqrt{3}) / 2, w=(1+i \sqrt{3}) / 2$ e $i$. Estes são estabilizados pelos subgrupos cíclicos finitos gerados respectivamente por $z \mapsto(-z-1) / z, z \mapsto(z-1) / z \mathrm{e} z \mapsto 1 / z$. Como $z \mapsto z+1$ leva $z$ a $w$ estes dois vértices pertencem ao mesmo ciclo elíptico. Como não existem outros vértices de $F$ cujo ângulo é $\leq 2 \pi / 3$ estes dois vértices formam um ciclo elíptico. O ponto $i$ é fixado por um elemento elíptico de ordem 2.

Como não existe outro ponto da fronteira de $F$ fixado por um elemento elíptico de 
ordem 2, $\{i\}$ é um ciclo elíptico consistindo de apenas um vértice. Pelo Teorema 1.90 , o grupo modular tem duas classes de conjugação de subgrupos cíclicos finitos maximais, um consistindo de grupos de ordem 2 e outro consistindo de grupos de ordem 3.

Definição 1.92. As ordems dos subgrupos finitos maximais de $\Gamma$ são chamados os períodos de $\Gamma$.

Proposição 1.93. Seja F uma região fundamental para o grupo Fuchsiano $\Gamma$. Sejam $\theta_{1}, \theta_{2}, \ldots \theta_{t}$ os ângulos em um conjunto de vértices congruentes de F. Seja $m$ a ordem do estabilizador em $\Gamma$ de um destes vértices. Então

$$
\theta_{1}+\theta_{2}+\ldots+\theta_{t}=2 \pi / m
$$

Prova: Sejam $v_{1}, v_{2}, \ldots, v_{t}$ os vértices pertencendo ao conjunto congruente, onde o ângulo interno em $v_{i}$ é $\theta_{i}$. Seja $H=\left\{I, S, S^{2}, \ldots, S^{m-1}\right\}$ o estabilizador de $v_{1}$ em $\Gamma$. Então todas as regiões $S^{r}(F)(0 \leq r \leq m-1)$ têm um vértice em $v_{1}$ cujo ângulo é $\theta_{1}$. Agora suponhamos que $T_{k}\left(v_{k}\right)=v_{1}\left(T_{k} \in \Gamma\right)$. Então o conjunto de todos os elementos que levam $v_{k}$ a $v_{1}$ é somente a classe lateral $H T_{k}$, assim todas as regiões $S^{r} T_{k}(F)$ têm a $v_{1}$ como um vértice, sendo seu ângulo $\theta_{k}$. Por outra parte, se uma região $A(F)(A \in \Gamma)$ tem um vértice em $v_{1}$, então $A^{-1}\left(v_{1}\right) \in F$ e assim $A^{-1}\left(v_{1}\right)=v_{i}$ para algum $i(1 \leq i \leq t)$. Logo $A \in H T_{i}$ e assim a região $A(F)$ foi incluída na descrição acima. Essas $m t$ regiões circundam o vértice $v_{1}$, e cada ângulo $\theta_{i}$ é repetido $m$ vezes; essas regiões são diferentes, pois se $S^{r} T_{k}(F)=S^{q} T_{l}(F)$ então $S^{r} T_{k}=S^{q} T_{l}$ e daí $T_{k}=T_{l}$ e $S^{r}=S^{q}$. Portanto

$$
m\left(\theta_{1}+\theta_{2}+\ldots+\theta_{t}\right)=2 \pi
$$




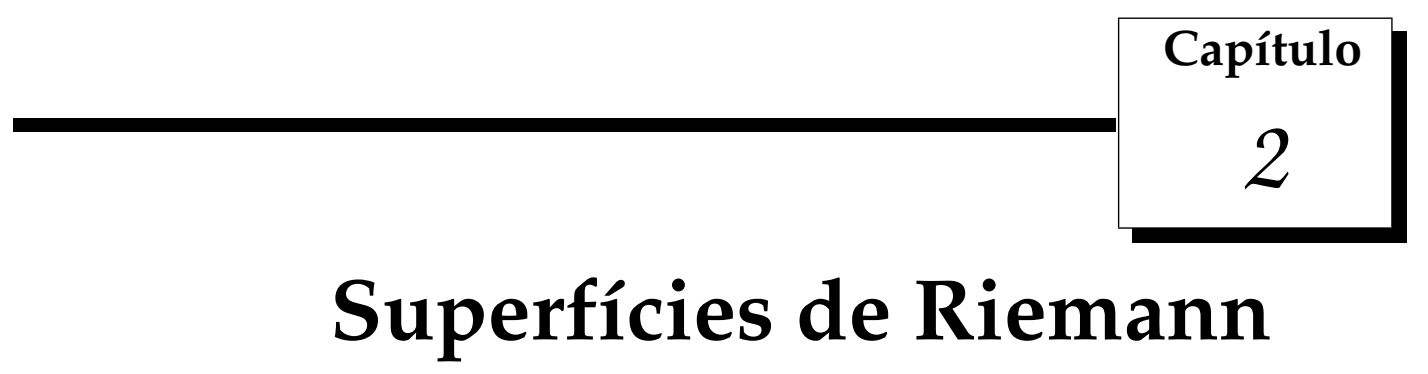

Neste capítulo vamos desenvolver alguns resultados básicos da teoria das superfícies de Riemann. O leitor pode ver [5],[8],[9] e [12] para um estudo mais profundo.

\subsection{Definição e Exemplos}

Lembrar que existe uma identificação natural de $\mathbb{R}^{2 n}$ com $\mathbb{C}^{n}$ dada pela correspondência $(x, y) \mapsto x+i y \operatorname{com} x, y \in R^{n}$.

Seja $U$ um subconjunto aberto de $\mathbb{C}^{n}$, dizemos que uma função $f=\left(f_{1}, \ldots, f_{m}\right)$ : $U \rightarrow \mathbb{C}^{m}$ é holomorfa em $U$, se para cada $i=1, \ldots, n, f_{i}: U \rightarrow \mathbb{C}$ é uma função holomorfa com respeito a cada uma de suas $n$ variáveis.

Agora, seja $S$ uma variedade topológica $2 n$-dimensional. Dizemos que um atlas $\mathcal{A}$ de $S$ é holomorfo se, considerando as mudanças de coordenadas como $n$ funções complexas em $n$ variavéis complexas (pela identificação acima), as mudanças de coordenadas são funções holomorfas.

Definição 2.1. Seja $S$ uma variedade topológica $2 n$-dimensional. Uma estrutura complexa n-dimensional em $S$, é um atlas holomorfo maximal $\mathcal{A}$ em $S$ e o par $(S, \mathcal{A})$ é chamada uma variedade complexa n-dimensional.

Em particular, temos que uma variedade complexa $n$-dimensional é uma variedade diferenciável de classe $C^{\infty} 2 n$-dimensional.

Definição 2.2. Uma superfície de Riemann é um par $(S, \mathcal{A})$, onde $S$ é uma variedade topológica 2-dimensional e $\mathcal{A}$ é uma estrutura complexa em $S$. 
De agora em diante, somente nos centraremos em superfícies de Riemann e escreveremos: $S$ uma superfície de Riemann em vez $(S, \mathcal{A})$ para indicar que $S$ tem alguma estrutura complexa dada.

A continuação algumas superfícies de Riemann são apresentadas.

Exemplo 2.3. (i) O plano complexo $\mathbb{C}$. Sua estrutura complexa é definida pelo atlas cuja única carta é $I d: \mathbb{C} \rightarrow \mathbb{C}$.

(ii) Domínios. Seja $R$ uma superfície de Riemann e $Y$ um domínio ${ }^{1}$ em $R$. Sua estrutura complexa é induzida pelas cartas $\varphi: U \rightarrow V$ em $R$ tais que $U \subset Y$.

Em particular o semiplano superior $\mathbb{H}$ é uma superfície de Riemann.

(iii) A esfera de Riemann $\widehat{\mathbb{C}}:=\mathbb{C} \cup\{\infty\}$. Introduzimos a seguinte topología em $\widehat{\mathbb{C}}$ : os conjuntos abertos são os abertos usuais $U \subset \mathbb{C}$ junto com conjuntos da forma $V \cup\{\infty\}$, onde $V \subset \mathbb{C}$ é o complemento de um conjunto compacto $K \subset \mathbb{C}$. Com esta topologia, $\widehat{\mathbb{C}}$ é um espaço de Hausdorff e compacto homeomorfo à esfera $S^{2}$. Sejam $U_{1}:=\widehat{\mathbb{C}} \backslash\{\infty\}=\mathbb{C}$ e $U_{2}:=\widehat{\mathbb{C}} \backslash\{0\}=\mathbb{C}^{*} \cup\{\infty\}$.

Definimos as aplicações $\varphi_{i}: U_{i} \rightarrow \mathbb{C}, i=1,2$ da seguinte maneira: $\varphi_{1}$ é a identidade e

$$
\varphi_{2}(z):= \begin{cases}1 / z & \text { para } z \in \mathbb{C}^{*} \\ 0 & \text { para } z=\infty\end{cases}
$$

Com estas cartas $\widehat{\mathbb{C}}$ é uma variedade topológica conexa de dimensão 2. A estrutura complexa em $\widehat{\mathbb{C}}$ é definido pelo atlas $\left\{\left(U_{i}, \varphi_{i}\right)\right\}_{i=1,2}$, pois $\varphi_{1}\left(U_{1} \cap U_{2}\right)=\varphi_{2}\left(U_{1} \cap U_{2}\right)=\mathbb{C}^{*}$ e $\varphi_{2} \circ \varphi_{1}^{-1}: \mathbb{C}^{*} \rightarrow \mathbb{C}^{*}$ dado por $z \mapsto 1 / z$ é biholomorfo.

(iv) Toro: Suponhamos que $w_{1}, w_{2} \in \mathbb{C}$ são linealmente independentes sobre $\mathbb{R}$. Definamos

$$
\Gamma:=\mathbb{Z} w_{1}+\mathbb{Z} w_{2}=\left\{n w_{1}+m w_{2} \mid n, m \in \mathbb{Z}\right\}
$$

$\Gamma$ é chamado a reticulado gerado por $w_{1}$ e $w_{2}$. Dois números complexos $z, z^{\prime} \in \mathbb{C}$ são ditos ser equivalentes sob $\Gamma$, ou módulo $\Gamma(\bmod \Gamma)$, se $z-z^{\prime} \in \Gamma$. O conjunto de todas as classes de equivalências é denotado por $\mathbb{C} / \Gamma$. Seja $\pi: \mathbb{C} \rightarrow \mathbb{C} / \Gamma$ a projeção canônica e introduzimos em $\mathbb{C} / \Gamma$ a topologia quociente sob $\pi$. Com esta topologia, $\pi$ é contínua e $\mathbb{C} / \Gamma$ é um espaço de Hausdorff conexo. Além disso $\mathbb{C} / \Gamma$ é compacto, pois é coberto pela imagem sob $\pi$ do paralelogramo compacto $P=\left\{\lambda w_{1}+\mu w_{2} \mid \lambda, \mu \in[0,1]\right\}$. Também $\pi$ é uma aplicação aberta (pois se $V \subset \mathbb{C}$ é aberto, então $\pi^{-1}(\pi(V))=\bigcup_{w \in \Gamma}(w+\Gamma)$ é aberto).

A estrutura complexa em $\mathbb{C} / \Gamma$ é definida da seguinte maneira: seja $V \subset \mathbb{C}$ um conjunto

\footnotetext{
${ }^{1}$ Seja $X$ um espaço topológico, dizemos que $Y \subset X$ é um domínio se $Y$ é um subconjunto aberto e conexo.
} 
aberto tal que não existem dois pontos equivalentes sob $\Gamma$ em $V$. Então $U=\pi(V)$ é um conjunto aberto e $\pi \mid V: V \rightarrow U$ é um homeomorfismo, e consideramos sua inversa $\varphi: U \rightarrow V$ como uma carta. Seja $\mathcal{A}$ o atlas obtido desta maneira, vejamos que para quaisquer duas cartas $\varphi_{i}: U_{i} \rightarrow V_{i}, i=1,2$ que pertencem a $\mathcal{A}$ a mudança de coordenadas é holomorfa. Com efeito, considerando $\psi=\varphi_{2} \circ \varphi_{1}^{-1}: \varphi_{1}\left(U_{1} \cap U_{2}\right) \rightarrow$ $\varphi_{2}\left(U_{1} \cap U_{2}\right)$, temos que $\pi(\psi(z))=\varphi_{1}^{-1}(z)=\pi(z)$ para todo $z \in \varphi_{1}\left(U_{1} \cap U_{2}\right)$ e assim $\psi(z)-z \in \Gamma$. Como $\Gamma$ é discreto em $\mathbb{C}$ e $\psi$ é contínua, isto implica que $\psi(z)-z$ é constante em cada componente conexa de $\varphi_{1}\left(U_{1} \cap U_{2}\right)$. Portanto $\psi$ é holomorfa.

Definição 2.4. Sejam $R$ e $S$ duas superfícies de Riemann. Dizemos que uma aplicação $f$ : $R \rightarrow S$ é holomorfa se $\psi \circ f \circ \varphi^{-1}: \varphi(U) \rightarrow \psi(V)$ é holomorfa para todas as cartas locais $(U, \varphi)$ de $R e(V, \psi)$ de $S$ com $f(U) \subset V$. Uma aplicação $f: R \rightarrow S$ é um biholomorfismo se $f$ é uma aplicação holomorfa com inversa $f^{-1}: S \rightarrow R$ holomorfa.

Observação 2.5. 1.- Sejam $\Gamma$ e $\Gamma^{\prime}$ dois reticulados e seja $\alpha \in \mathbb{C}^{*}$, então a aplicação $\mathbb{C} \rightarrow$ $\mathbb{C}, z \mapsto \alpha z$ induz uma aplicação holomorfa $\tilde{\alpha}: \mathbb{C} / \Gamma \rightarrow \mathbb{C} / \Gamma^{\prime}$ o qual é biholomorfismo se e somente se $\alpha \Gamma=\Gamma^{\prime}$.

2.- Da observação anterior, temos que todo toro $\mathbb{C} / \Gamma$ é biholomorfo a um toro da forma $R_{\tau}:=\mathbb{C} / \Gamma_{\tau}$, onde $\Gamma_{\tau}$ é um reticulado da forma $\mathbb{Z}+\tau \mathbb{Z}$ para algum $\tau \in \mathbb{H}$.

3.- Utilizando as equações de Cauchy-Riemann pode-se ver que toda superfície de Riemann é orientável.

Nas notações do Teorema 1.19 , temos a seguinte definição.

Definição 2.6. Uma superfície de Riemann homeomorfa a um dos $S_{g}$ é chamada uma superfície de Riemann fechada de gênero $g$.

A esfera de Riemann é de gênero 0 e um toro é de gênero 1. Do Teorema 1.19, temos que toda superfície de Riemann compacta é uma superfície de Riemann fechada de gênero finito.

Uma superfície de Riemann não compacta é chamada uma superfície de Riemann aberta.

Definição 2.7. O espaço de moduli $\mathcal{M}_{g}$ de gênero g é o espaço das classes de equivalências de superfícies de Riemann fechadas de gênero $g$, onde duas superfícies de Riemann fechadas $R e$ $S$ de gênero $g$ são equivalentes, se existe um biholomorfismo $f: R \rightarrow S$.

\subsection{Teorema de Uniformização}

Nesta seção, apresentamos o Teorema de uniformização devido a Klein, Poincaré e Koebe o qual classifica as superfícies de Riemann simplesmente conexas, a menos de 
biholomorfismo. Este teorema tem muitas consequências importantes, as quais apresentaremos algumas neste capítulo. Para o leitor interesado na prova deste teorema, uma excelente referência é [5, pág.210].

Definição 2.8. Sejam $R$ e $\widetilde{R}$ duas superfícies de Riemann. Uma aplicação holomorfa sobrejetora $\pi: \widetilde{R} \rightarrow R$ é chamada uma aplicação de recobrimento se todo ponto $p$ de $R$ tem uma vizinhança $U$ tal que para cada componente conexa $V$ da imagen inversa $\pi^{-1}(U)$ de $U$ a restrição $\pi \mid V: V \rightarrow U$ é um biholomorfismo.

Dizemos que $(\widetilde{R}, \pi, R)$ é um recobrimento de $R$, e $\widetilde{R}$ uma superfície de recobrimento de $R$. A aplicação de recobrimento $\pi$ é chamada também a projeção de $\widetilde{R}$ sobre $R$. Além disso, quando $\widetilde{R}$ é simplesmente conexa, $(\widetilde{R}, \pi, R)$ é chamado um recobrimento universal de $R$, e $\widetilde{R}$ uma superfície de recobrimento universal de $R$.

Exemplo 2.9. 1. Seja $\pi: \mathbb{C} \rightarrow \mathbb{C}-\{0\}$ dado por $\pi(z)=e^{z}$. Então $\mathbb{C}$ é uma superfície de recobrimento universal de $\mathbb{C}-\{0\}$.

2. Seja $\pi: \mathbb{H} \rightarrow \Delta-\{0\}$ dado por $\pi(z)=e^{2 \pi i z}$. Então $\mathbb{H}$ é uma de superfície de recobrimento universal de $\Delta-\{0\}$

3. Seja $\pi: \mathbb{C}-\{0\} \rightarrow \mathbb{C}-\{0\}$ dado por $\pi(z)=z^{n}$, onde $n$ é un inteiro positivo. Então $\mathbb{C}-\{0\}$ é uma superfície de recobrimento sobre se mesmo.

Definição 2.10. Uma aplicação biholomorfa $\gamma: \widetilde{R} \rightarrow \widetilde{R}$ satisfazendo $\pi \circ \gamma=\pi$ é chamada uma transformação do recobrimento $(\widetilde{R}, \pi, R)$. Para um recobrimento $(\widetilde{R}, \pi, R)$ dado, denotamos por $\Gamma$ o conjunto de todas suas tranformações do recobrimento. $\Gamma$ é um grupo, com a operação de composição de aplicações, chamado grupo de transformações do recobrimento de $(\widetilde{R}, \pi, R)$. Em particular, chamamos de $\Gamma$ o grupo de transformações do recobrimento universal se $\widetilde{R}$ é uma superfície de recobrimento universal de $R$.

Exemplo 2.11. Damos os grupos de transformções de recobrimento dos recobrimentos do Exemplo 2.9

$\Gamma=\left\langle\gamma_{1}\right\rangle \operatorname{com} \gamma_{1}(z)=z+2 \pi i$.

$\Gamma=\left\langle\gamma_{1}\right\rangle \operatorname{com} \gamma_{1}(z)=z+1$.

$\Gamma=\left\langle\gamma_{1}\right\rangle \operatorname{com} \gamma_{1}(z)=z \exp (2 \pi i / n)$ o qual é un grupo finito de ordem $n$.

Seja $(\widetilde{R}, \pi, R)$ um recobrimento de uma superfície de Riemann $R$. Um ponto $\widetilde{p}$ em $\widetilde{R}$ esta sobre um ponto $p$ em $R$ se $\pi(\widetilde{p})=p$. Um levantamento de um caminho $C$ em $R$ é um caminho $\widetilde{C}$ em $\widetilde{R} \operatorname{com} \pi \circ \widetilde{C}=C$

Proposição 2.12. Toda superfície de Riemann $R$ tem um recobrimento universal $(\widetilde{R}, \pi, R)$. 
Prova: Fixemos um ponto base $p_{0}$ em $R$. Seja um par $(C, p)$ de qualquer ponto $p$ em $R$ e qualquer caminho $C$ em $R$ de $p_{0}$ a $p$. Dois pares $(C, p)$ e $\left(C^{\prime}, p^{\prime}\right)$ são equivalentes se $p=p^{\prime}$ e $C$ é homotópico a $C^{\prime}$ em $R$. Denotamos por $[C, p]$ a classe de equivalência de $(C, p)$. Seja $\widetilde{R}$ o conjunto de todas a classes de equivalência $[C, p]$.

$\widetilde{R}$ é uma superfície de Riemann:

Para qualquer ponto $\widetilde{p}=[C, p]$ de $\widetilde{R}$, tomamos uma vizinhança $U_{p}$ de $p$ o qual é um domínio simplesmente conexo em $R$. Seja

$$
U_{\widetilde{p}}=\left\{\left[C \cdot C_{q}, q\right] \mid q \in U_{p} \text { e } C_{q}(\mathrm{I}) \subset U_{p} \operatorname{com} C_{q}(0)=p, C_{q}(1)=q\right\}
$$

Desde que $U_{p}$ é um domínio simplesmente conexo, temos uma correspondência $1-1$ entre $U_{p}$ e $U_{\widetilde{p}}$. Esses $U_{\widetilde{p}}$ definem um sistema fundamental de vizinhanças de $\widetilde{p}$ em $\widetilde{R}$. Então $\widetilde{R}$ resulta um espaço topológico de Hausdorff. Seja $\pi: \widetilde{R} \rightarrow R$ a projeção dada por $\pi([C, p])=p$, pela construção temos que $\pi$ é uma aplicação contínua de $\widetilde{R}$ sobre $R$ e satisfaz a condição de aplicação de recobrimento.

Dado um ponto $\widetilde{p}=[C, p]$, tomamos uma vizinhanca coordenada $\left(U_{p}, z_{p}\right)$ tal que $U_{p}$ é um domínio simplesmente conexo, então a família $\left\{\left(U_{\widetilde{p}}, z_{\widetilde{p}}\right)\right\}$, onde $z_{\widetilde{p}}=z \circ \pi$ define uma estrutura complexa em $\widetilde{R}$.

Seja $I_{0}$ o caminho constante $I_{0}(t)=p_{0}$ para qualquer $t \in \mathrm{I}$. Para provar a conexidade de $\widetilde{R}$, é suficiente mostrar que todo ponto $[C, p] \in \widetilde{R}$ é conectado com $\left[I_{0}, p_{0}\right]$ por um caminho em $\widetilde{R}$. Para cada $s \in$ I definimos um caminho $C_{s}$ em $R$ dado por $C_{s}(t)=C(s t)$ para todo $t \in \mathrm{I}$. Pondo $\widetilde{C}(s)=\left[C_{s}, C(s)\right]$ para todo $s \in \mathrm{I}$, temos um caminho $\widetilde{C}$ em $\widetilde{R}$ de $\left[I_{0}, p_{0}\right]$ a $[C, p]$, o que implica que $\widetilde{R}$ é conexo.

$\widetilde{R}$ é simplesmente conexo:

É suficiente mostrar que todo caminho fechado $\widetilde{C}$ em $\widetilde{R}$ com ponto base $\left[I_{0}, p_{0}\right]$ é homotópico a $\left[I_{0}, p_{0}\right]$. Seja $C=\pi \circ \widetilde{C}$. Então $C$ é um caminho fechado em $R$ com ponto base $p_{0}$, e o ponto final de $\widetilde{C}$ é representado por $\left[C, p_{0}\right]$. Desde que $\widetilde{C}$ é um caminho fechado, temos que $\left[I_{0}, p_{0}\right]=\left[C, p_{0}\right]$, o que significa que $C$ é homotópico a $I_{0}$. Seja $F: \mathrm{I} \times \mathrm{I} \rightarrow R$ uma homotopia entre elas tal que $\left\{F_{s}=F(\cdot, s)\right\}_{s \in \mathrm{I}}$ satisfaz $F_{0}=I_{0}$, $F_{1}=C$, e $F_{s}(0)=F_{s}(1)=p_{0}$ para qualquer $s \in \mathrm{I}$. Então, para qualquer $(t, s) \in \mathrm{I} \times \mathrm{I}$, definimos um caminho $C_{(t, s)}$ em $R$ dado por $C_{(t, s)}(u)=F_{s}(t u)$ para todo $u \in$ I. Logo, temos uma homotopia $\widetilde{F}(t, s): \mathrm{I} \times \mathrm{I} \rightarrow \widetilde{R}$ entre $\widetilde{C}$ e $\left[I_{0}, p_{0}\right]$ pondo $\widetilde{F}(t, s)=\left[C_{(t, s)}, F_{s}(t)\right]$ para todo $(s, t) \in \mathrm{I} \times \mathrm{I}$. Portanto, $\widetilde{R}$ é simplesmente conexo.

Teorema 2.13 (Teorema de Uniformização). Toda superfície de Riemann $R$ simplesmente conexa, é biholomorficamente equivalente a uma das seguintes três superfícies de Riemann: $\widehat{\mathbb{C}}, \mathbb{C}$ ou $\mathbb{H}$. 
Como consequência imediata do Teorema 2.12 e do Teorema de uniformização temos:

Teorema 2.14. Para toda superfície de Riemann $R$, existe um recobrimento universal $\widetilde{R}$ de $R$ o qual é biholomorfo a um das seguintes três superfícies de Riemann: $\widehat{\mathbb{C}}, \mathbb{C}$ ou $\mathbb{H}$.

Vamos listar algumas propriedades conhecidas das aplicações de recobrimento, as quais serãos utilizadas posteriormente.

Lema 2.15 (Existência e unicidade do levantamento de um caminho). Ver [11, pág.123] Para qualquer caminho $C$ em $R$ com ponto inicial p e para qualquer ponto $\widetilde{p} \in \widetilde{R}$ sobre p, existe um único levantamento $\widetilde{C}$ de $C$ com ponto inicial $\widetilde{p}$.

Teorema 2.16 (Levantamento de uma aplicação). Para superfícies de Riemann $R$ e $S$, sejam $\left(\widetilde{R}, \pi_{R}, R\right)$ e $\left(\widetilde{S}, \pi_{S}, S\right)$ seus respectivos recobrimentos universais construídos anteriormente. Então, dada uma aplicação contínua $f: R \rightarrow S$, existe uma aplicação contínua $\widetilde{f}: \widetilde{R} \rightarrow \widetilde{S} \operatorname{com} f \circ \pi_{R}=\pi_{S} \circ \widetilde{f}$. Esta $\widetilde{f}$ é determinada unicamente sob a condição $\widetilde{f}\left(\widetilde{p}_{1}\right)=\widetilde{q}_{1}$, onde $\widetilde{p}_{1} \in \widetilde{R}$ e $\widetilde{q}_{1} \in \widetilde{S}$ são tais que $\pi_{S}\left(\widetilde{q}_{1}\right)=f\left(\pi_{R}\left(\widetilde{p}_{1}\right)\right)$.

Além disso, se fé diferenciável ou holomorfa, então f também é diferenciável ou holomorfa.

Prova: Sejam $\widetilde{p}_{1}=\left[C_{1}, p_{1}\right]$ e $\widetilde{q}_{1}=\left[D_{1}, f\left(p_{1}\right)\right]$, obtemos uma aplicação definida por $\widetilde{f}([C, p])=\left[D_{1} \cdot f\left(C_{1}\right)^{-1} \cdot f(C), f(p)\right]$ para todos os pontos $[C, p] \in \widetilde{R}$. Então tem-se que $\widetilde{f}\left(\widetilde{p}_{1}\right)=\widetilde{q}_{1}$ e $f \circ \pi_{R}=\pi_{S} \circ \widetilde{f}$, como $\pi_{R}$ e $\pi_{S}$ são localmente biholomorfismos e $f$ é contínua (difeomorfismo ou holomorfa), então $\widetilde{f}$ deve ser contínua (difeomorfismo ou holomorfa). A unicidade segue-se do Lema 2.15 .

Teorema 2.17 (Unicidade do recobrimento universal). Ver [11, pág.132].

Para quaisquer dois recobrimentos universais $(\widetilde{R}, \pi, R)$ e $\left(\widetilde{R}_{1}, \pi_{1}, R\right)$ da superfície de Riemann $R$ existe um biholomorfismo $\varphi: \widetilde{R} \rightarrow \widetilde{R}_{1}$ tal que $\pi_{1} \circ \varphi=\pi$.

Dada uma superfície de Riemann $R$, denotamos por Aut $(R)$ o grupo de biholomorfismos de $R$.

Dado um recobrimento universal $(\widetilde{R}, \pi, R)$, sabemos que seu grupo de transformações $\Gamma$ é um subgrupo de $A u t(\widetilde{R})$. Por causa do teorema de uniformização, se queremos conhecer $\Gamma$, precisamos conhecer os grupos $A u t(\widehat{\mathbb{C}}), A u t(\mathbb{C})$ e $A u t(\mathbb{H})$.

Lema 2.18. (i) $\operatorname{Aut}(\widehat{\mathbb{C}})=P S L(2, \mathbb{C})$.

(ii) $\operatorname{Aut}(\mathbb{C})=\{z \mapsto a z+b \mid a, b \in \mathbb{C}, a \neq 0\}$.

(iii) $\operatorname{Aut}(\mathbb{H})=P S L(2, \mathbb{R})$. 
Prova: $(i)$ Se $\gamma(\infty)=\infty$. Então em uma vizinhança de $\infty, \gamma$ tem a expansão de Laurent

$$
\gamma(z)=a z+\sum_{n=0}^{\infty} b_{n} z^{-n}
$$

onde $a \neq 0$. Então $\gamma(z)-a z$ é holomorfo em $\widehat{\mathbb{C}}$, daqui o princípio do máximo mostra que $\gamma(z)-a z$ deve ser uma função constante, digamos $b$. Assim, temos que $\gamma(z)=a z+b$ com $a \neq 0$. Se $\gamma\left(z_{0}\right)=z_{0} \neq \infty$, pondo $\gamma_{1}(z)=1 /\left(z-z_{0}\right)$, vemos que $\gamma_{1}$ e $\gamma_{1} \circ \gamma$ são elementos de $\operatorname{Aut}(\widehat{\mathbb{C}})$, e $\gamma_{1} \circ \gamma(\infty)=\infty$. Assim, temos $\gamma_{1} \circ \gamma(z)=1 /\left(\gamma(z)-z_{0}\right)=a_{1} z+b_{1}$, onde $a_{1}, b_{1} \in \mathbb{C}$ com $a_{1} \neq 0$.

(ii) Todo elemento $\gamma \in A$ ut $(\mathbb{C})$ é extendido a um elemento de $A u t(\widehat{\mathbb{C}})$ pondo $\gamma(\infty)=\infty$. Pelo argumento $(i)$ segue-se afirmação $(i i)$.

(iii) Sabemos que $\Delta$ e $\mathbb{H}$ são biholomorfos, $T: \mathbb{H} \rightarrow \Delta$ dado por $T(z)=(z-i) /(z+i)$ é o biholomorfismo. Se $\gamma \in A u t(\Delta)$, seja $\gamma(0)=\beta$, então a tranformação de Möbius $\gamma_{1}(z)=(z-\beta) /(1-\bar{\beta} z)$ pertence a $\operatorname{Aut}(\Delta)$. Daqui $\gamma_{2}=\gamma_{1} \circ \gamma$ também pertence a Aut $(\Delta)$ e $\gamma_{2}(0)=0$, o lema de Schwarz implica que $\gamma_{2}=e^{i \theta} z$ para algum $\theta \in \mathbb{R}$. Logo, se $\gamma \in A u t(\mathbb{H})$ então $\gamma_{1}=T \circ \gamma \circ T^{-1} \in A u t(\Delta)$ é uma tranformação de Möbius, da forma $(a z+b) /(c z+d), a, b, c, d \in \mathbb{C}, a d-b c=1$. Devido a que $\gamma$ mapea $\mathbb{H}$ sobre $\mathbb{H}$, pode-se assumir que $a, b, c, d \in \mathbb{R}$ e assim $\gamma \in P S L(2, \mathbb{R})$.

O seguinte resultado nos va permitir identificar $\mathcal{M}_{1}$.

Teorema 2.19. Para quasiquer dois pontos $\tau, \tau^{\prime} \in \mathbb{H}$ os toros $R_{\tau}$ e $R_{\tau^{\prime}}$ são biholomorfos se e somente $\tau$ e $\tau^{\prime}$ satisfazem a relação

$$
\tau^{\prime}=\frac{a \tau+b}{c \tau+d}
$$

onde $a, b, c, d$ são inteiros satisfazendo $a d-b c=1$.

Prova: Assumamos que existe um biholomorfismo $f: R_{\tau^{\prime}} \rightarrow R_{\tau}$, seja $\tilde{f}: \mathbb{C} \rightarrow \mathbb{C}$ tal que $\pi_{\tau} \circ \tilde{f}=f \circ \pi_{\tau^{\prime}}$, isto é, o levantamento de $f$, o qual é biholomorfismo pois $f$ é biholomorfismo (Ver Teorema 2.16), logo $\tilde{f}$ escreve-se como $\widetilde{f}(z)=\alpha z+\beta$, onde $\alpha, \beta \in \mathbb{C}$ e $\alpha \neq 0$ (Ver Lema 2.18). Podemos assumir que $\widetilde{f}(0)=0$ e daí $\beta=0$. Assim $\widetilde{f}\left(\tau^{\prime}\right)$ e $\widetilde{f}(1)$ são equivalentes a $\widetilde{f}(0)=0$ sob $\Gamma_{\tau}$. Portanto

$$
\begin{array}{r}
\widetilde{f}\left(\tau^{\prime}\right)=\alpha \tau^{\prime}=a \tau+b \\
\widetilde{f}(1)=\alpha=c \tau+d
\end{array}
$$

onde $a, b, c, d$ são inteiros. Portanto, obtemos a relação (2.1). Aplicando o mesmo argumento para $\tilde{f}^{-1}$ obtemos

$$
\tau=\frac{a^{\prime} \tau^{\prime}+b^{\prime}}{c^{\prime} \tau^{\prime}+d^{\prime}}
$$


onde $a^{\prime}, b^{\prime}, c^{\prime}, d^{\prime}$ são inteiros. Além disso, das relações $\widetilde{f}^{-1} \circ \widetilde{f}(1)=1$ e $\widetilde{f}^{-1} \circ \widetilde{f}(\tau)=\tau$, vemos que $a d-b c= \pm 1$. Desde que

$$
\operatorname{Im} \tau^{\prime}=\frac{a d-b c}{|c \tau+d|^{2}}(\operatorname{Im} \tau)>0
$$

temos que $a d-b c=1$.

Reciprocamente, se (2.1) cumpre-se, então um biholomorfismo $f: R_{\tau^{\prime}} \rightarrow R_{\tau}$ é dado por $f([z])=[(c \tau+d) z]$.

Do Teorema 2.19 e o item 2 da Obeservação 2.5 temos que $\mathcal{M}_{1}$ é identificado com o quociente de $\mathbb{H}$ por $P S L(2, \mathbb{Z})$, isto é, $\mathcal{M}_{1} \cong \mathbb{H} / P S L(2, \mathbb{Z})$.

Dado um recobrimento universal $(\widetilde{R}, \pi, R)$ de uma superfície de Riemann $R$, veremos que seu grupo de recobrimento universal $\Gamma$ é isomorfo ao grupo fundamental $\pi_{1}\left(R, p_{0}\right)$ de $R$.

Para qualquer elemento $\left[C_{0}\right] \in \pi_{1}\left(R, p_{0}\right)$, definimos a ação $\left[C_{0}\right]_{*}$ em $\widetilde{R}$ por

$$
\left[C_{0}\right]_{*}([C, p])=\left[C_{0} \cdot C, p\right], \quad[C, p] \in \widetilde{R}
$$

Tem-se que $\left[C_{0}\right]_{*} \in \Gamma$. Com estas notações temos o seguinte teorema.

Teorema 2.20. A correspondência $\phi: \pi_{1}\left(R, p_{0}\right) \rightarrow \Gamma$ dada por $\left[C_{0}\right] \mapsto\left[C_{0}\right]_{*}$ é um isomorfismo.

Prova: Da definição segue-se que $\phi$ é um homomorfismo.

$\phi$ é injetora: Suponhamos que $\left[C_{0}\right]_{*}$ é o elemento unitário de $\Gamma$. Então temos

$$
\left[C_{0}\right]_{*}\left(\left[I_{0}, p_{0}\right]\right)=\left[C_{0}, p_{0}\right]=\left[I_{0}, p_{0}\right]
$$

onde $I_{0}$ é o caminho constante $p_{0}$. Assim $C_{0}$ é homotópico a $I_{0}$, e daí $\left[C_{0}\right]$ é o elemento unitário de $\pi_{1}\left(R, p_{0}\right)$.

$\phi$ é sobrejetora: Dado $\gamma \in \Gamma$, tomamos o caminho $\widetilde{C}$ em $\widetilde{R}$ desde $\left[I_{0}, p_{0}\right]$ a $\gamma\left(\left[I_{0}, p_{0}\right]\right)$. A relação $\pi \circ \gamma=\pi$ implica que $C=\pi \circ \widetilde{C}$ é um caminho fechado em $R$ com ponto base $p_{0}, \operatorname{assim} \widetilde{C}$ é um levantamento de $C$ e $\left[I_{0}, p_{0}\right]$ e $\left[C, p_{0}\right]$ são os pontos inicial e final de $\widetilde{C}$ respectivamente. Desde que $[C] \in \pi_{1}\left(R, p_{0}\right)$, Teorema 2.16 implica que $\gamma=[C]_{*}$.

Lema 2.21. O grupo $\Gamma$ do recobrimento universal de $(\widetilde{R}, \pi, R)$ de uma superfície de Riemann $R$ satisfaz:

(i) Para quaisquer $\widetilde{p}, \widetilde{q} \in \widetilde{R} \operatorname{com} \pi(\widetilde{p})=\pi(\widetilde{q})$, existe um $\gamma \in \Gamma \operatorname{com} \widetilde{q}=\gamma(\widetilde{p})$.

(ii) Para qualquer $\widetilde{p} \in \widetilde{R}$, existe uma vizinhança $\widetilde{U}$ de $\widetilde{p}$ em $\widetilde{R}$ tal que $\gamma(\widetilde{U}) \cap \widetilde{U}=\emptyset$ para todo $\gamma \in \Gamma-\{I d\}$. Em particular, cada elemento diferente da identidade não tem pontos fixos. 
(iii) Para qualquer subconjunto compacto $K$ de $\widetilde{R}$ existem ao máximo un número finito de elementos $\gamma \in \Gamma$ tal que $\gamma(K) \cap K \neq \emptyset$, isto é, $\Gamma$ age propriamente descontínua em $\widetilde{R}$.

Prova: (i) Suponhamos que $\pi(\widetilde{p})=\pi(\widetilde{q})=p$, então temos que $\widetilde{p}=\left[C_{1}, p\right]$ e $\widetilde{q}=\left[C_{2}, p\right]$ para alguns caminhos $C_{1}$ e $C_{2}$ em $R$. Pondo $C_{0}=C_{2} \cdot C_{1}^{-1}$ temos que $\gamma=\left[C_{0}\right]_{*}$ satisfaz $\widetilde{q}=\gamma(\widetilde{p})$.

(ii) Tomamos um ponto $\widetilde{p} \in \widetilde{R}$, e seja $p=\pi(\widetilde{p}), \widetilde{p}=[C, p]$. Escolhamos uma vizinhança $U$ de $p$ em $R$ satisfazendo a condição da definição de aplicação de recobrimento e denotamos por $\widetilde{U}$ a componente conexa de $\pi^{-1}(U)$ contendo $\widetilde{p}$. Se $\gamma(\widetilde{U}) \cap \widetilde{U} \neq \emptyset$ para algum $\gamma \in \Gamma$, existem pontos $\widetilde{p}_{1}, \widetilde{q}_{1} \in \widetilde{U} \operatorname{com} \widetilde{q}_{1}=\gamma\left(\widetilde{p}_{1}\right)$. Desde que $\pi \circ \gamma=\pi$, obte$\operatorname{mos} \pi\left(\widetilde{p}_{1}\right)=\pi\left(\widetilde{q}_{1}\right)$ e portanto $\widetilde{p}_{1}=\widetilde{q}_{1}$, pois $\pi$ é biholomorfismo em $\widetilde{U}$. Assim temos $\gamma\left(\widetilde{p}_{1}\right)=\operatorname{Id}\left(\widetilde{p}_{1}\right)$, logo pelo Teorema 2.16, podemos concluir que $\gamma=I d$.

(iii) Assumamos que existe uma sequência $\left\{\gamma_{n}\right\}_{n=1}^{\infty}$ consistindo de elementos diferentes de $\Gamma$ tal que $\gamma_{n}(K) \cap K \neq \emptyset$ para todo $n$. Então para cada $n$, encontramos pontos $\widetilde{q}_{n}, \widetilde{r}_{n} \in K \operatorname{com} \widetilde{r}_{n}=\gamma_{n}\left(\widetilde{q}_{n}\right)$, logo pela compacidade de $K$ podemos assumir que $\left\{\widetilde{q}_{n}\right\}_{n=1}^{\infty},\left\{\widetilde{r}_{n}\right\}_{n=1}^{\infty}$ convergem a $\widetilde{q}_{0}, \widetilde{r}_{0} \in K$, respectivamente, quando $n \rightarrow \infty$. Como $\pi \circ \gamma_{n}=\pi$, obtemos $\pi\left(\widetilde{q}_{n}\right)=\pi\left(\widetilde{r}_{n}\right)$ e $\pi\left(\widetilde{q}_{0}\right)=\pi\left(\widetilde{r}_{0}\right)$. Tomamos uma vizinhança $U$ de $\pi\left(\widetilde{q}_{0}\right)$ em $R$ satisfazendo a condição da definição de aplicação de recobrimento, e denotamos por $\widetilde{U}$ e $\widetilde{V}$ as componentes conexas de $\pi^{-1}(U)$ contendo $\widetilde{q}_{0}$ e $\widetilde{r}_{0}$, respectivamente. Desde que $\left\{\gamma_{n}\left(\widetilde{q}_{n}\right)\right\}_{n=1}^{\infty}$ converge a $\widetilde{r}_{0}$, temos $\gamma_{n}(\widetilde{U}) \cap \widetilde{U} \neq \emptyset$ para $n$ suficientemente grande, $\pi \circ \gamma_{n}(\widetilde{U})=U$ implica $\gamma_{n}(\widetilde{U})=\widetilde{V}$, isto é, $\gamma_{n+1}^{-1} \circ \gamma_{n}(\widetilde{U})=\widetilde{U}$. Logo $(i i)$ implica que $\gamma_{n+1}=\gamma_{n}$, o qual é uma contradição.

Proposição 2.22. Seja $\pi: \widetilde{R} \rightarrow R$ um recobrimento universal da superfície de Riemann $R$, então $\pi$ induz uma bijeção natural

$$
\Phi: \Gamma / \operatorname{Int}(\Gamma) \rightarrow \tilde{\Pi}_{1}(R)
$$

onde Int $(\Gamma)$ é o grupo de automorfismos internos de $\Gamma$

Prova: Fixemos $\tilde{x} \in \widetilde{R}$. Dado $T \in \Gamma$, seja $\tilde{\alpha}$ o caminho que junta $\tilde{x} \operatorname{com} T(\tilde{x})$ e seja $\alpha=\pi \circ \alpha$. Denotaremos por $[T]$ a classe de equivalência $T$ sob $\operatorname{Int}(\Gamma)$ e por $[\alpha]$ a classe de homotopia de $\alpha$ sob homotopia livre. Assim, temos a função requerida dada por $\Phi:[T] \mapsto[\alpha]$ e é bem definida.

A inversa é construída da seguinte maneira: dado um laço $\alpha \in R$, tomamos $\tilde{x} \in$ $\pi^{-1}(\alpha(0))$ e levantamos $\alpha$ para um caminho $\tilde{\alpha}$ empezando em $\tilde{x}$, logo existe um único $T \in \Gamma$ tal que $\tilde{\alpha}(1)=T(\tilde{x})$. E assim obtemos a função bem definida $\Psi:[\alpha] \mapsto[T]$ e pode-se ver que é a inversa de $\Phi$. 
Dado $(\widetilde{R}, \pi, R)$ um recobrimento universal de uma superfície de Riemann $R$, dizemos que dois pontos $\widetilde{p}, \widetilde{q} \in \widetilde{R}$ são $\Gamma$-equivalentes, se existe um elemento $\gamma \in \Gamma$ tal que $\widetilde{q}=\gamma(\widetilde{p})$. Denotamos por $[\widetilde{p}]$ a classe de equivalência de $\widetilde{p}$ e por $\widetilde{R} / \Gamma$ o conjunto de todas estas classes de equivalência. Tem-se uma projeção canônica $\pi_{\widetilde{R}}: \widetilde{R} \rightarrow \widetilde{R} / \Gamma$, a topologia de $\widetilde{R} / \Gamma$ é a topología quociente respeito de $\pi_{\widetilde{R}}$.

Teorema 2.23. Seja $(\widetilde{R}, \pi, R)$ um recobrimento universal de uma superfície de Riemann $R$ com $\Gamma$ seu grupo do recobrimento universal. Então $\widetilde{R} / \Gamma$ é uma superfície de Riemann a qual é biholomorfa a $R$.

Prova: É claro que $\widetilde{R} / \Gamma$ é conexo. Para ver que $\widetilde{R} / \Gamma$ é Hausdorff utiliza-se a propiedade (iii) do Lema 2.21. Agora, definimos uma estrutura complexa em $\widetilde{R} / \Gamma$ como segue: para qualquer ponto $\widetilde{p} \in \widetilde{R}$, tomamos uma vizinhança $\widetilde{U}_{\widetilde{p}}$ satisfazendo a propiedade (ii) do Lema 2.21 e podemos assumir que existe uma coordenada local $z_{\widetilde{p}}$ em $\widetilde{U}_{\widetilde{p}}$. Pondo $p=\pi_{\widetilde{R}}(\widetilde{p}), U_{p}=\pi_{\widetilde{R}}\left(\widetilde{U}_{p}\right)$, vemos que $\pi_{\widetilde{R}}: \widetilde{U}_{p} \rightarrow U_{p}$ é um homeomorfismo. Portanto, pondo $z_{p}=z_{\widetilde{p}} \circ \pi_{\widetilde{R}}^{-1}$, concluímos que $\left\{\left(U_{p}, z_{p}\right)\right\}_{p \in \widetilde{R} / \Gamma}$ define uma estrutura complexa tal que $\left(\widetilde{R}, \pi_{\widetilde{R}}, \widetilde{R} / \Gamma\right)$ é um recobrimento de $\widetilde{R} / \Gamma$. O biholomorfismo $\phi: \widetilde{R} / \Gamma \rightarrow R$ é dado por $[\widetilde{p}] \mapsto \pi(\widetilde{p})$.

O Teorema 2.23 mostra que toda superfície de Riemann $R$ pode-se representar pela superfície de Riemann quociente $\widetilde{R} / \Gamma$, de algum recobrimento universal $(\widetilde{R}, \pi, R)$ de $R$ por seu grupo de transformações $\Gamma$ do recobrimento universal .

No que resta desta seção, dada uma superfície de Riemann $R$, vamos determinar sob que condições em $R$ seu recobrimento universal $\widetilde{R}$ é biholomorfo a: $\widehat{\mathbb{C}}$ ou $\mathbb{C}$ ou $\mathbb{H}$.

Teorema 2.24. Uma superfície de Riemann $R$ tem recobrimento universal $\widetilde{R}$ biholomorfo à esfera de Riemann $\widehat{\mathbb{C}}$ se e somente se Ré biholomorfa a $\widehat{\mathbb{C}}$.

Prova: Suponhamos que $\widetilde{R}=\widehat{\mathbb{C}}$. Desde que todo elemento $\gamma$ de seu grupo de recobrimento universal $\Gamma$ é uma transformação de Möbius, ele deve ter pontos fixos. Do Lema 2.21, qualquer $\gamma \in \Gamma-\{I d\}$ não tem pontos fixos em $\widehat{\mathbb{C}}$. Portanto $\Gamma=\{I d\}$, o que implica que $R=\widetilde{R} / \Gamma=\widehat{\mathbb{C}}$.

Reciprocamente, se $R=\widehat{\mathbb{C}}$, como $\widehat{\mathbb{C}}$ é simplesmente conexa, pela construção de $\widetilde{R}$ segue-se que $\widetilde{R}=\widehat{\mathbb{C}}$.

Lema 2.25. Seja $\Gamma$ um subgrupo de Aut $(\mathbb{H})$ tal que todo elemento de $\Gamma-\{I d\}$ não tem pontos fixos em $\mathbb{H}$ e tal que a ação de $\Gamma$ é propriamente descontínua em $\mathbb{H}$. Se $\Gamma$ é abeliano, então é cíclico.

Prova: Tomamos um elemento $\gamma_{0} \in \Gamma \operatorname{com} \gamma \neq I d$. Desde que $\gamma_{0}$ não tem pontos fixos em $\mathbb{H}$, então temos que $\gamma_{0}$ é parabólico ou hiperbólico. 
Se $\gamma_{0}$ é parabólico, podemos assumir que $\gamma_{0}(z)=z+b_{0}\left(b_{0} \in \mathbb{R}, b_{0} \neq 0\right)$ por conjugação em $A u t(\mathbb{H})$. Logo, temos que $\gamma \in A u t(\mathbb{H})$ é commutativo com $\gamma_{0}$ se e somente se $\gamma$ pode-se escrever como $\gamma(z)=z+b$ para algum $b \in \mathbb{R}$. Desde que $\Gamma$ age propriamente descontínua em $\mathbb{H}$, existe um número positivo $b_{1}$ tal que $\Gamma$ é gerado por $\gamma_{1}(z)=z+b_{1}$. Se $\gamma_{0}$ é hiperbólico, podemos assumir que $\gamma_{0}(z)=\lambda_{0} z\left(\lambda_{0}>0\right)$ por conjugação em Aut $(\mathbb{H})$. Logo, temos que $\gamma \in A u t(\mathbb{H})$ é commutativo com $\gamma_{0}$ se e somente se $\gamma(z)=\lambda z$ para algum número positivo $\lambda$. Desde que $\Gamma$ age propriamente descontínua em $\mathbb{H}$, existe um número positivo $\lambda_{1}$ tal que $\Gamma$ é gerado por $\gamma_{1}(z)=\lambda_{1} z$.

Teorema 2.26. Uma superfície de Riemann $R$ tem um recobrimento universal biholomorfo a $\mathbb{C}$ se e somente se $R$ é biholomorfa a $\mathbb{C}, \mathbb{C}-\{0\}$ ou a um toro.

Prova: Suponhamos que $\widetilde{R}=\mathbb{C}$. Seja $\Gamma$ seu grupo de recobrimento universal, desde que $\Gamma$ é um subgrupo de $A u t(\mathbb{C})$, pelo Lema 2.18, todo $\gamma \in \Gamma$ é da forma $\gamma(z)=a z+$ $b(a, b \in \mathbb{C}, a \neq 0)$ e $a=1$ pois qualquer $\gamma \in \Gamma-\{I d\}$ não tem pontos fixos em $\mathbb{C}$. Como $\Gamma$ age propriamente descontínua em $\mathbb{C}$ (Lema 2.21), pode ocurrir um dos seguintes casos:

(i) $\Gamma=\{I d\}$.

(ii) $\Gamma=\left\langle\gamma_{0}\right\rangle$, onde $\gamma_{0}(z)=z+b_{0}$ para algum $b_{0} \in \mathbb{C}-\{0\}$.

(iii) $\Gamma=\left\langle\gamma_{0}, \gamma_{1}\right\rangle$, onde $\gamma_{0}(z)=z+b_{0}$ e $\gamma_{1}(z)=z+b_{1}$ para alguns $b_{0}, b_{1} \in \mathbb{C}$ as quais são linealmente independentes sobre $\mathbb{R}$.

Portanto, nos casos $(i),(i i)$ e $(i i i)$ a superfície de Riemann $\widetilde{R}=\mathbb{C} / \Gamma$ é biholomorfa a $\mathbb{C}, \mathbb{C}-\{0\}$ ou a um toro, respectivamente.

Reciprocamente, se $R=\mathbb{C}$ obtemos $\widetilde{R}=\mathbb{C}$ pois $\mathbb{C}$ é simplesmente conexo. Agora, suponhamos $R=\mathbb{C}-\{0\}$ então obtemos $\widetilde{R}=\mathbb{C}$ (item 1 do Exemplo 2.9.).

Finalmente, suponhamos que $R$ é um toro. Se $\widetilde{R}$ é biholomorfo a $\mathbb{H}$, então $\Gamma$ deve ser um grupo livre abeliano com dois geradores, mas isto é uma contradição ao Lema 2.25 . Portanto $\widetilde{R}$ é biholomorfa a $\mathbb{C}$.

Como consequência do Teorema 2.24 e Teorema 2.26, temos o seguinte resultado.

Corolário 2.27. Toda superfície de Riemann Ré biholomorfa a uma das seguintes: $\mathbb{C}, \mathbb{C}-\{0\}$, um toro, $\widehat{\mathbb{C}}$ ou a $\mathbb{H} / \Gamma$, onde $\Gamma$ é um subgrupo de $\operatorname{Aut}(\mathbb{H})=P S L(2, \mathbb{R})$ agindo propriamente descontínua e livremente ${ }^{2}$ em $\mathbb{H}$.

Em particular, se $R$ uma superfície de Riemann fechada de gênero $g \geq 2$ então $\widetilde{R}$ é biholomorfo a $\mathbb{H}$ e $R$ é biholomorfa a $\mathbb{H} / \Gamma$, esta expressão sera denotada por $R=\mathbb{H} / \Gamma$, para algum subgrupo $\Gamma$ de $P S L(2, \mathbb{R})$ agindo propriamente descontínua e livremente

\footnotetext{
${ }^{2}$ A ação de um grupo $G$ sobre um conjunto $X$ é livre se o conjunto $G_{x}=\{g \in G \mid g \cdot x=x\}$ contem unicamente o elemento neutro $\{e\}$ do grupo $G$.
} 
em $\mathbb{H}$. Do Teorema 1.68 tal subgrupo $\Gamma$ é um grupo Fuchsiano. Além disso, tal $\Gamma$ não tem elementos elípticos (a ação é livre) e neste caso dizemos que $\Gamma$ é um modelo Fuchsiano para $R$.

\subsection{O quociente $\mathbb{H} / \Gamma$}

Dada uma superfície de Riemann fechada $R$ de gênero $g \geq 2$ vimos que $R=\mathbb{H} / \Gamma$, onde $\Gamma$ é um modelo Fuchsiano para $R$.

O seguinte resultado mostra que os modelos Fuchsianos para $R$ são conjugados.

Teorema 2.28. Sejam $\Gamma_{1}, \Gamma_{2}$ grupos Fuchsianos sem elementos elípticos. Então $\mathbb{H} / \Gamma_{1} e \mathbb{H} / \Gamma_{2}$ são superfícies de Riemann biholomorfas se e somente se existe $T \in P S L(2, \mathbb{R})$ tal que $T \Gamma_{1} T^{-1}=$ $\Gamma_{2}$

Prova: Como $\Gamma_{1}, \Gamma_{2}$ agem sem pontos fixos, então as aplicações $\pi_{i}: \mathbb{H} \rightarrow \mathbb{H} / \Gamma_{i}, i=1,2$ são aplicações de recobrimento.

Se existe um biholomorfismo $g: \mathbb{H} / \Gamma_{1} \rightarrow \mathbb{H} / \Gamma_{2}$, então pelo Teorema 2.16 existe um biholomorfismo $T=\tilde{g}: \mathbb{H} \rightarrow \mathbb{H}, \tilde{g} \in P S L(2, \mathbb{R})$ tal que o seguinte diagrama commuta.

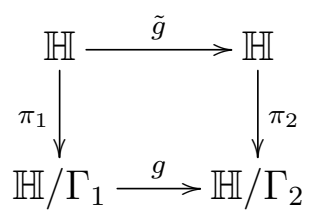

isto é, $g\left([z]_{\Gamma_{1}}\right)=[T(z)]_{\Gamma_{2}}$. Agora, se $S \in \Gamma_{1}$, então $[z]_{\Gamma_{1}}=[S(z)]_{\Gamma_{1}}$ e portanto $[T S(z)]_{\Gamma_{2}}=$ $[T(z)]_{\Gamma_{2}}$. Logo existe $V \in \Gamma_{2}$ tal que $T S(z)=S T(z)$ para todo $z \in \mathbb{H}$ e assim $T S T^{-1}=$ $V \in \Gamma_{2}$. Portanto $T \Gamma_{1} T^{-1} \subset \Gamma_{2}$. Um argumento similar, utilizando $g^{-1}\left([z]_{\Gamma_{2}}\right)=$ $\left[T^{-1}(z)\right]_{\Gamma_{1}}$ mostra que $T^{-1} \Gamma_{2} T \subset \Gamma_{1}$.

Reciprocamente, se $T \Gamma_{1} T^{-1}=\Gamma_{2}$ então a aplicação $[z]_{\Gamma_{1}} \mapsto[T(z)]_{\Gamma_{2}}$ é um biholomorfismo de $\mathbb{H} / \Gamma_{1}$ sobre $\mathbb{H} / \Gamma_{2}$.

O seguinte resultado mostra que superfícies de Riemann também podem ser obtidas de $\mathbb{H}$ mediante grupos Fuchsianos (se $\Gamma$ não tem elementos elípticos o resultado é o Teorema 2.23.

Teorema 2.29. Ver [8, pág.248].

Seja $\Gamma$ um grupo Fuchsiano qualquer, então $\mathbb{H} / \Gamma$ tem estrutura de superfície de Riemann tal que a projeção canônica $\pi: \mathbb{H} \rightarrow \mathbb{H} / \Gamma$ é holomorfa.

Agora, apresentamos resultados que relacionam o quociente $\mathbb{H} / \Gamma$ com a região fundamental de $\Gamma$. 
Teorema 2.30. Se F é uma região de Dirichlet para um grupo Fuchsiano $\Gamma$, então $F / \Gamma$ é homeomorfo a $\mathbb{H} / \Gamma$.

Prova: Seja $i: F \rightarrow \mathbb{H}$ a inclusão e $\psi: F \rightarrow F / \Gamma, \pi: \mathbb{H} \rightarrow \mathbb{H} / \Gamma$ as projeçoes canônicas contínuas. Definimos $\theta: F / \Gamma \rightarrow \mathbb{H} / \Gamma$ como a aplicação tal que satisfaz $\theta(\psi(z))=\pi(z)$. Se $\psi\left(z_{1}\right)=\psi\left(z_{2}\right)$, então $z_{2}=S\left(z_{1}\right)$ para algum $S \in \Gamma$ e assim $\pi\left(z_{1}\right)=\pi\left(z_{2}\right)$. Portanto, $\theta$ é bem definida e bijetora. Agora, se $V \subset \mathbb{H} / \Gamma$ é aberto então $\pi^{-1}(V)$ é aberto em $\mathbb{H}$ e assim $F \cap \pi^{-1}(V)$ é aberto em $F$. Da igualdade $\psi^{-1}\left(\theta^{-1}(V)\right)=F \cap \pi^{-1}(V)$ obtemos que $\theta^{-1}(V)$ é aberto em $F / \Gamma$. Portanto $\theta$ é contínua. Vejamos que $\theta$ é aberta.

Seja $A \subset F / \Gamma$ um conjunto aberto e seja $[z]=\psi(z) \in A$. Pelo Teorema 1.89 , $F$ é localmente finito, assim temos que $\psi^{-1}([z])=\left\{z=T_{0}(z), T_{1}(z), \ldots, T_{s}(z)\right\}$ é um conjunto finito. Como $\psi^{-1}(A)=\tilde{A}$ é relativamente aberto em $F$ e $\psi^{-1}([z])$ é finito, existe um disco hiperbólico $B$ com centro $z$ tal que

$$
T_{j}(B) \cap F \subset \tilde{A} \text { e } T(B) \cap F \neq \emptyset \text { implica que } T=T_{j}(1 \leq j \leq s)
$$

Temos que, $\theta([z])=\pi(z) \in \pi(B)$ o qual é aberto.

Afirmação: $\pi(B) \subset \theta(A)$. De fato, seja $[w] \in \pi(B), w \in B$. Então existe $T \in \Gamma$ tal que $T(w) \in F$. Logo $T(B) \cap F \neq \emptyset$ e por (2.3) temos $T=T_{j}$ para algum $j=1, \ldots, s$. Portanto $T_{j}(w) \in T_{j}(B)$ e $T_{j}(w) \in F$ e por $[2.3), T_{j}(w) \in \tilde{A}$. Logo,

$$
[w]=\pi(w)=\pi\left(T_{j}(w)\right) \in \pi(\tilde{A})=\pi \circ i(\tilde{A})=\theta(A)
$$

e assim tem-se a afirmação.

Portanto $\theta(A)$ é uma vizinhança aberta de $\theta([z])$.

Teorema 2.31. Seja F uma região de Dirichlet para um grupo Fuchsiano $\Gamma$. Então $\mathbb{H} / \Gamma$ é compacto se e somente se F é um subconjunto compacto de $\mathbb{H}$.

Prova: Do Teorema 2.30 resulta que se $F$ é compacto então $\mathbb{H} / \Gamma$ é compacto.

Suponhamos agora que $\mathbb{H} / \Gamma$ é compacto. Pelo Teorema 1.89 , segue-se que se $[z] \in \mathbb{H} / \Gamma$ então existem ao máximo um número finito de $z^{\prime} \in F$ tal que $\pi\left(z^{\prime}\right)=[z]$. Agora, seja $\left\{z_{i}\right\}_{i=1}^{n}$ uma sequência de pontos diferentes em $F$.

Mostraremos que esta sequência tem um ponto límite em $F$. Com efeito, a sequência $\left\{\left[z_{i}\right]\right\}_{i=1}^{n}$ tem um ponto límite $[l] \in \mathbb{H} / \Gamma$, $[l]$ tem um número finito de pre-imagens $l_{1}, \ldots, l_{r}$ em $F$.

Afirmação: Algum dos pontos $l_{i}, i=1, \ldots, r$ que um destes pontos é um ponto límite de $\left\{z_{i}\right\}_{i=1}^{n}$. De fato, caso contrário cada $l_{i}$ tem uma vizinhança $V_{i}$ em $\mathbb{H}$ contendo só um 
número finito desta sequência e como $l_{1}, \ldots, l_{r}$ estão na mesma $\Gamma$-órbita, cada $V_{i}$ contém um número finito de pontos da forma $S\left(z_{j}\right)$. Logo $\bigcap_{i=1}^{r} \pi(V)$ é uma vizinhança aberta de $[l]$ contendo somente um número finito da sequência $\left\{\left[z_{i}\right]\right\}_{i=1}^{n}$, o que é uma contradição.

Portanto $F$ é compacto.

Observação 2.32. Seja $\Gamma$ um grupo Fuchsiano tal que $\mathbb{H} / \Gamma$ é compacto. Então pelo Teorema 2.31, qualquer região de Dirichlet é compacta. Portanto $F$ tem um número finito de lados e assim somente um número finito de ciclos elípticos. Pelo Teorema 1.90. $\Gamma$ tem um número finito de períodos $m_{1}, \ldots, m_{r}$. Dizemos que $\Gamma$ tem assinatura $\left(g ; m_{1}, \ldots, m_{r}\right)$ se $\mathbb{H} / \Gamma$ tem gênero $g$ e $m_{1}, \ldots, m_{r}$ são os períodos de $\Gamma$.

Teorema 2.33. Seja $\Gamma$ com assinatura $\left(g ; m_{1}, \ldots, m_{r}\right)$. Se $F$ é uma região fundamental para $\Gamma$ cuja fronteira tem área hiperbólica zero, então a área hiperbólica de $F$ é

$$
\mu(F)=2 \pi\left[(2 g-2)+\sum_{i=1}^{r}\left(1-\frac{1}{m_{i}}\right)\right]
$$

Prova: Temos que $F$ tem $r$ ciclos elípticos (como na Seção 1.4, incluimos o ponto interior de um lado fixado por um elemento elíptico de ordem 2 como um vértice cujo ângulo é $\pi$ e este lado é considerado sendo composto de dois lados separados por este vértice). Pelo Teorema 1.93 a soma dos ângulos em todos vértices elípticos é $\sum_{i=1}^{r} \frac{2 \pi}{m_{i}}$. Suponhamos que existem outros $s$ ciclos de vértices, desde que a ordem dos estabilizadores destes vértices e 1 . Logo, a soma dos ângulos nestes vértices é $2 \pi s$. Assim a soma de todos os ângulos de $F$ é igual a

$$
2 \pi\left[\left(\sum_{i=1}^{r} \frac{1}{m_{i}}\right)+s\right]
$$

Os lados de $F$ são emparelhados por elementos de $\Gamma$. Se identificamos esses lados emparelhados, obtemos uma superfície compacta orientável de gênero $g$. Se $F$ tem $n$ destes lados identificados, obtemos uma decomposição de $\mathbb{H} / \Gamma$ em $r+s$ vértices, $n$ arestas, e 1 face simplesmente conexa. Logo, pela fórmula de Euler,

$$
2-2 g=(r+s)-n+1
$$


Daí, pelo Corolário 1.47, obtemos

$$
\mu(F)=(2 n-2)-2 \pi\left[\left(\sum_{i=1}^{r} \frac{1}{m_{i}}\right)+s\right]=2 \pi\left[(2 g-2)+\sum_{i=1}^{r}\left(1-\frac{1}{m_{i}}\right)\right]
$$

O seguinte resultado, utilizando o Teorema 2.33, proporciona uma cota inferior para a $\mu(F)$ sob algumas condições.

Proposição 2.34. Ver [8 pág.261]. Se F é uma região de Dirichlet para um grupo Fuchsiano tal que $\mathbb{H} / \Gamma$ é compacto, então $\mu(F) \geq \pi / 21$. Se $\mu(F)=\pi / 21$, então $\Gamma$ é um grupo triangular ${ }^{3}$ com assinatura $(0 ; 2,3,7)$.

O seguinte teorema é o recíproco do Teorema 2.33 .

Teorema 2.35. Se $g \geq 0, m_{i} \geq 2$ são inteiros e se

$$
2 g-2+\sum_{i=1}^{r}\left(1-\left(1 / m_{i}\right)\right)>0
$$

então existe um grupo Fuchsiano com assinatura $\left(g ; m_{1}, \ldots, m_{r}\right)$.

Prova: Vamos utilizar o modelo do dico $\Delta$ da geometria hiperbólica (neste caso as $\mathbb{H}-$ linhas são os círculos e linhas Euclideanas ortogonais à fronteira de $\Delta$ ). Do centro de $\Delta$ traçamos $4 g+r$ raios com ângulos iguais. Seja $0<t<1$ e escolhamos pontos a distância Euclideana $t$ do centro ao longo de cada raio. Se juntamos estes pontos sucessivos por $\mathbb{H}$-linhas, obtemos um polígono hiperbólico $M(t)$. Nos primeiros $r$ lados de $M(t)$ construímos $r$ triângulos hiperbólicos isósceles externos tal que os ângulos entre os lados iguais sejam $2 \pi / m_{1}, \ldots, 2 \pi / m_{r}$ (o triângulo será degenerado quando $m_{i}=2$, na Figura 2.1 temos $g=2, r=4, m_{i}>2$, para $i=1,2,3$ e $\left.m_{4}=2\right)$. A união de $M(t)$ com estes triângulos é um polígono hiperbólico $N(t)$ com $4 g+2 r$ lados. Denotamos seus lados por $\alpha_{1}, \beta_{1}^{\prime}, \alpha_{1}^{\prime}, \beta_{1}, \ldots, \alpha_{g}, \beta_{g}^{\prime}, \alpha_{g}^{\prime}, \beta_{g}, \xi_{1}, \xi_{1}^{\prime}, \ldots, \xi_{r}, \xi_{r}^{\prime}$ e os orientamos como na Figura 2.1 .

Agora, a área hiperbólica de $N(t) \rightarrow 0$ quando $t \rightarrow 0$ e pelo Corolário $1.47 N(t) \rightarrow$ $(4 g+2 r-2) \pi-\sum_{i=1}^{r} 2 \pi / m_{i}$ quando $t \rightarrow 1$.

Daqui, para algum $t_{0}$ a área hiperbólica de $N\left(t_{0}\right)$ é

$$
2 \pi\left[(2 g-2)+\sum_{i=1}^{r}\left(1-\frac{1}{m_{i}}\right)\right]
$$

\footnotetext{
${ }^{3}$ Ver [8, pág.238].
} 
Por construção, $\alpha_{i}, \alpha_{i}^{\prime}$ têm o mesmo comprimento hiperbólico como também $\beta_{i}, \beta_{i}^{\prime}$ e

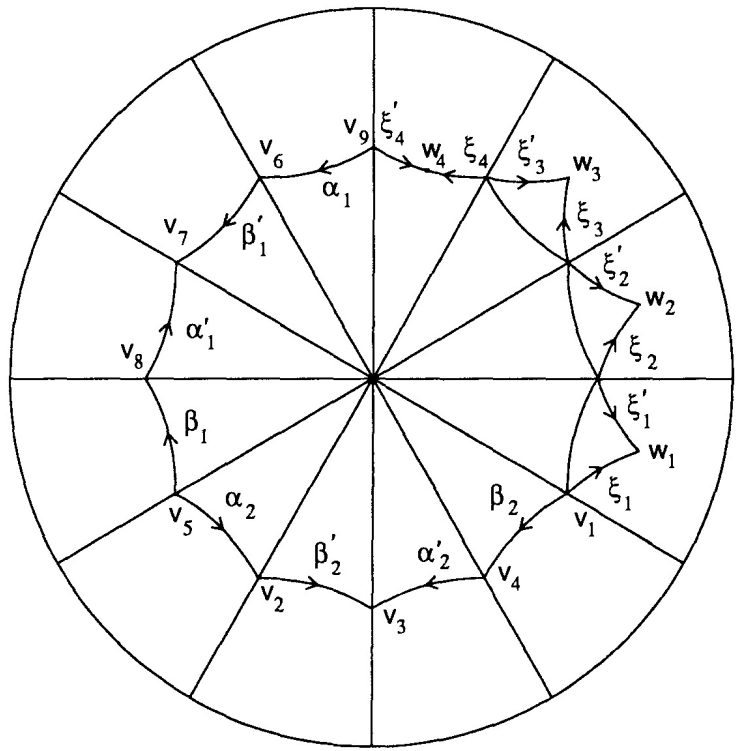

Figura 2.1: $M(t)$

$\xi_{i}, \xi_{i}^{\prime}$. Daqui, existem isometrias hiperbólicas $A_{i}, B_{j}, X_{k}(i, j=1, \ldots, g ; k=1, \ldots, r)$ tal que

$$
A_{i}\left(\alpha_{i}^{\prime}\right)=\alpha_{i}, B_{j}\left(\beta_{j}^{\prime}\right)=\beta_{j}, X_{k}\left(\xi_{k}^{\prime}\right)=\xi_{k}
$$

Agora, calculamos as classes de congruência dos vértices. Começamos chamando ao vértice inicial de $\beta_{g}, v_{1}$. Este é congruente (por $B_{g}^{-1}$ ) ao vértice inicial de $\beta_{g}^{\prime}, v_{2}$, o qual é também o vértice final de $\alpha_{g}$ e é congruente (por $A_{g}^{-1}$ ) ao vértice final de $\alpha_{g}^{\prime}, v_{3}$, o qual é também o vértice final de $\beta_{g}^{\prime}$ a qual é congruente (por $B_{g}$ ) ao vértice final de $\beta_{g}, v_{4}$. $\mathrm{O}$ vértice $v_{4}$ é o vértice inicial de $\alpha_{g}^{\prime}$ que é congruente (por $A_{g}$ ) ao vértice inicial de $\alpha_{g}$, que é também o vértice inicial de $\beta_{g-1}$ (Ver Figura 2.1). Continuando com este processo, vemos que todos os vértices do polígono original $M\left(t_{0}\right)$ formam um conjunto congruente. Os outros $r$ vértices $w_{1}, \ldots, w_{r}$ formam $r$ conjuntos congruentes, cada um com um único elemento.

Seja $\Gamma$ o grupo gerado por $\left\{A_{i}, B_{j}, X_{k} \mid i, j=1, \ldots, g ; k=1, \ldots, r\right\}$. Como a área hiperbólica de $N\left(t_{0}\right)$ é $2 \pi\left\{(2 g-2)+\sum_{i=1}^{r}\left(1-\frac{1}{m_{i}}\right)\right\}$, então pelo Corolário 1.47 vemos que a soma dos ângulos nos vertices $v_{1}, \ldots, v_{4 g+r}$ é $2 \pi$.

As condições necessárias da Proposição 1.93 são satisfeitas: a soma dos ângulos em $v_{1}, \ldots, v_{4 g+r}$ é igual a $2 \pi$ e o ângulo em $w_{k}$ é igual a $2 \pi / m_{k}, k=1, \ldots, r$. Com estas condições pode-se ver que $N\left(t_{0}\right)$ é uma região fundamental para $\Gamma$, e se voltamos para $\mathbb{H}$, vemos que a $\Gamma$-órbita de cada ponto de $\mathbb{H}$ é um conjunto discreto e pelo Corolário 1.69. $\Gamma$ é um grupo Fuchsiano.

O espaço quociente $\Delta / \Gamma$ é decomposto em $r+1$ vértices (correpondentes a os $(r+1)$ 
conjuntos de vértices congruentes de $\left.N\left(t_{0}\right)\right),(2 g+r)$ arestas e simplesmente uma face. Então, pela fórmula de Euler, seu gênero $h$ satisfaz:

$$
2-2 h=(r+1)-(2 g+r)+1=2-2 g
$$

e portanto $h=g$. Existem $r$ ciclos elipticos, $\left\{w_{1}\right\}, \ldots,\left\{w_{r}\right\}$, e o estabilizador de cada $w_{i}$ tem ordem $m_{i}, i=1, \ldots, r$. Portanto, $\Gamma$ tem assinatura $\left(g ; m_{1}, \ldots, m_{r}\right)$.

\subsection{Métrica hiperbólica em superfícies de Riemann}

Seja uma superfície de Riemann $R$ cujo recobrimento universal é biholomorfo a $\mathbb{H}$ e consideremos $\Gamma$ um modelo Fuchsiano de $R$ agindo em $\mathbb{H}$. Seja $\pi: \mathbb{H} \rightarrow R$ a projeção de $\mathbb{H}$ sobre $R=\mathbb{H} / \Gamma$. Como a métrica de Poincaré $d s^{2}$ é invariante sob a ação de $\Gamma$, obtemos uma métrica Riemanniana $d s_{R}^{2}$ em $R$ a qual satisfaz $\pi^{*}\left(d s_{R}^{2}\right)=d s^{2}$. Chamamos a esta métrica $d s_{R}^{2}$ a métrica hiperbólica em $R$.

Teorema 2.36. Todo elemento de um modelo Fuchsiano de uma superfície de Riemann $R$ fechada de gênero $g \geq 2$ consiste somente da identidade e elementos hiperbólicos.

Prova: Como $R=\mathbb{H} / \Gamma$ e $\Gamma$ age sem pontos fixos, então $\Gamma$ não tem elementos elípticos. Seja $F$ a região de Dirichlet para $\Gamma$. Então, pelo Teorema 2.31, $F$ é compacto. Logo, pelo Corolário 1.67 temos que

$$
\eta(z)=\inf \{\rho(z, T(z)) \mid T \backslash\{I d\} \in \Gamma\}>0
$$

Como para cada $T \in \Gamma, \rho(z, T z)$ é uma função contínua de $z, \eta(z)$ é uma função contínua de $z$. Portanto, como $F$ é compacto, $\eta=\inf \{\eta(z) \mid z \in F\}$ é atingido e $\eta>0$. Se $z \in \mathbb{H}$ então existe $S \in \Gamma$ tal que $w=S(z) \in F$. Então, para $T_{0} \in \Gamma \backslash\{I d\}$ temos

$$
\rho\left(z, T_{0}(z)\right)=\rho\left(S(z), S\left(T_{0}(z)\right)\right)=\rho\left(w, S T_{0} S^{-1}(w)\right) \geq \eta
$$

e portanto

$$
\inf \{\rho(z, T(z)) \mid z \in \mathbb{H}\}=\eta>0
$$

Suponhamos agora que $\Gamma$ contém elementos parabólicos. Pelo Teorema 2.28, podemos supor que $T_{0}(z)=z+1$, mas isto é uma contradição, pois $\rho(z, z+1) \rightarrow 0$ quando $\operatorname{Im}(z) \rightarrow \infty$.

Proposição 2.37. Seja $R$ uma superfície de Riemann fechada de gênero $g \geq 2$, então cada classe de homotopia livre não trivial em $R$, tem um único representante geodésico. 
Prova: Seja $\alpha$ um laço homotopicamente não trivial, e seja $T \in \Phi^{-1}([\alpha])$ (T era definido tal que $T(\tilde{\alpha}(0))=\tilde{\alpha}(1)$ para algum levantamento de $\alpha$ e $\Phi$ é aplicação dada em (2.2) da Proposição 2.22). Pelo Teorema 2.36, $T$ é hiperbólico e seja $E_{T}$ o eixo de $T$. Logo, se $\pi: \mathbb{H} \rightarrow \mathbb{H} / \Gamma=R$ é a projeção, então $\gamma=\pi\left(E_{T}\right)$ é um laço geodésico em $R$ na mesma classe de homotopia livre de $\alpha$. Suponhamos que $\gamma_{1}$ é outro laço geodésico em $M$ na mesma classe de homotopia livre de $\alpha$ e seja $\tilde{\gamma}_{1}$ um levantamento de $\gamma_{1}$. Se $T^{\prime} \in \Phi^{-1}([\alpha])$ é tal que $T^{\prime}\left(\tilde{\gamma}_{1}(0)\right)=\tilde{\gamma}_{1}(1)$ então, o fato de que $\tilde{\gamma}_{1}$ é uma geodésica de $\mathbb{H}$ implica que é $T^{\prime}$-invariante. Além disso, $T^{\prime}=S^{-1} T S$ para algum $S$, daqui $\tilde{\gamma}_{1}=S^{-1}(\tilde{\gamma})$. Portanto $\gamma=\gamma_{1}$ e assim o representante geodésico é único.

Observação 2.38. O Teorema anterior diz que o representante geodésico $\gamma$ de uma classe de homotopia livre não trivial é a projeção do eixo $E_{T}$ de algum elemento hiperbólico $T$ de $\Gamma$, onde $R=\mathbb{H} / \Gamma$, ainda mais pode-se ver que se o laço é simples então o representante geodésico é simples.

Proposição 2.39. Seja $R$ uma superfície de Riemann de gênero fechada $g \geq 2$. Então o comprimento $l(\gamma)$ do representante geodésico de uma classe de homotopia não trivial satisfaz

$$
4 \cosh ^{2} \frac{l(\gamma)}{2}=(a+d)^{2}=\operatorname{tr}^{2}(T)
$$

onde $T=\frac{a z+b}{c z+d}, a d-b c=1$ é o elemento hiperbólico de um modelo Fuchsiano $\Gamma$ de $R$ tal que a projeção de $E_{T}$ é $\gamma$.

Prova: Como $l(\gamma)$ e $\operatorname{tr}(T)^{2}$ são invariantes sob a conjugação de $T$ por um elemento de $A u t(\mathbb{H})$, podemos assumir que $T(z)=\lambda z,(\lambda>1)$ e assim $a=\sqrt{\lambda}, b=c=0$ e $d=1 / \sqrt{\lambda}$. Neste caso temos

$$
l(\gamma)=\int_{1}^{\lambda} \frac{d y}{y}=\ln \lambda=2 \ln a
$$

Logo $\cosh ^{2}(\ln a)=\frac{1}{4} t^{2}(T)$ 


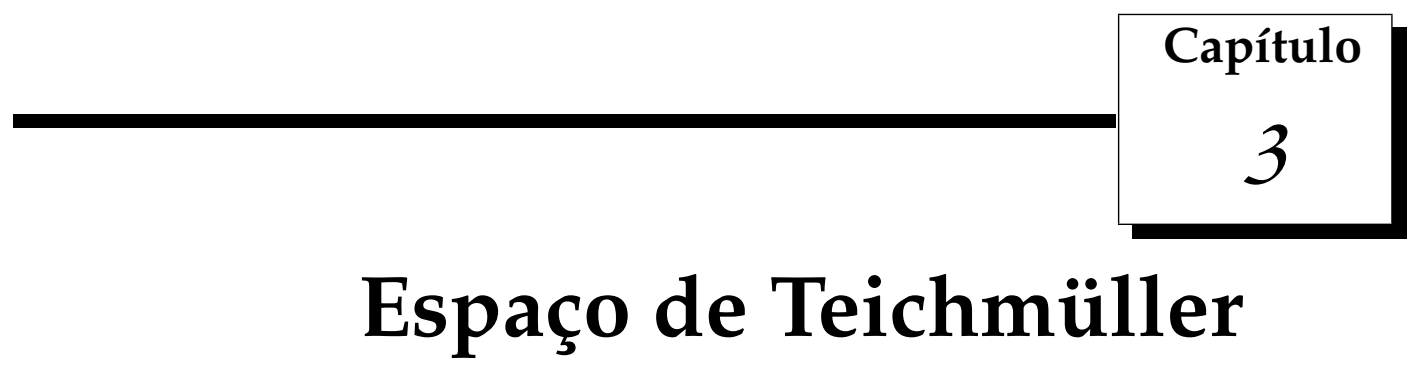

Neste capítulo vamos apresentar o espaço de Teichmüller de gênero $g \geq 1$ e as Coordenadas de Fricke para tal espaço no caso $g \geq 2$. Por último definimos a estrutura conforme de uma variedade Riemanniana e veremos sua relação com as estruturas complexas no caso 2-dimensional. As principais referências para este capítulo são [3],[7] e [9].

\subsection{Espaço de Teichmüller de gênero $\mathrm{g}$}

Definição 3.1. Seja $R$ uma superfície de Riemann fechada de gênero $g$. Uma marcação em $R$ é um sistema canônico de geradores $\Sigma_{p}=\left\{\left[A_{j}\right],\left[B_{j}\right]\right\}_{j=1}^{g}$ de $\pi_{1}(R, p)$.

Duas marcações $\Sigma_{p}=\left\{\left[A_{j}\right],\left[B_{j}\right]\right\}_{j=1}^{g}$ e $\Sigma_{p^{\prime}}=\left\{\left[A_{j}^{\prime}\right],\left[B_{j}^{\prime}\right]\right\}_{j=1}^{g}$ em $R$ são equivalentes se existe uma curva contínua $C_{0}$ em $R$ tal que $\left[A_{j}^{\prime}\right]=T_{C_{0}}\left(\left[A_{j}\right]\right)$ e $\left[B_{j}^{\prime}\right]=T_{C_{0}}\left(\left[B_{j}\right]\right)$ onde $T_{C_{0}}: \pi_{1}(R, p) \rightarrow \pi_{1}\left(R, p^{\prime}\right)$ é o isomorfismo que leva $[C]$ em $\left[C_{0}^{-1} \cdot C \cdot C_{0}\right]$.

Sejam $\Sigma_{p}$ e $\Sigma_{q}$ duas marcações nas superfícies de Riemann fechadas $R$ e $S$ de gênero $g$, respectivamente. Dizemos que $\left(R, \Sigma_{p}\right)$ e $\left(S, \Sigma_{q}\right)$ são equivalentes se existe um biholomorfismo $h: S \rightarrow R$ tal que a marcação $h_{*}\left(\Sigma_{q}\right)=\left\{h_{*}\left(\left[A_{j}^{\prime}\right]\right), h_{*}\left(\left[B_{j}^{\prime}\right]\right)\right\}_{j=1}^{g}$ é equivalente a $\Sigma_{p}=\left\{\left[A_{j}\right],\left[B_{j}\right]\right\}_{j=1}^{g}$.

Definição 3.2. O espaço de Teichmüller $T_{g}$ de gênero g é o conjunto de todas as classes de equivalência $\left[R, \Sigma_{p}\right]$.

O próximo Teorema nos va permitir identificar o espaço $T_{1}$. 
Teorema 3.3. Para todo ponto $\tau \in \mathbb{H}$, seja $\Sigma(\tau)=\left\{\left[A_{1}(\tau)\right],\left[B_{1}(\tau)\right]\right\}$ a marcação em $R_{\tau}=$ $\mathbb{C} / \Gamma_{\tau}$ para o qual $\left[A_{1}(\tau)\right]$ e $\left[B_{1}(\tau)\right]$ correspondem a 1 e $\tau$ em $\Gamma_{\tau}$, respectivamente. Então $\left[R_{\tau}, \Sigma(\tau)\right]=\left[R_{\tau^{\prime}}, \Sigma\left(\tau^{\prime}\right)\right]$ em $T_{1}$ se e somente se $\tau=\tau^{\prime}$.

Prova: Suponhamos que $\left[R_{\tau}, \Sigma(\tau)\right]=\left[R_{\tau^{\prime}}, \Sigma\left(\tau^{\prime}\right)\right]$. Então existe um biholomorfismo $h: R_{\tau^{\prime}} \rightarrow R_{\tau}$ tal que $h_{*}\left(\Sigma\left(\tau^{\prime}\right)\right)=\left\{h_{*}\left(\left[A_{1}\left(\tau^{\prime}\right)\right]\right), h_{*}\left(\left[B_{1}\left(\tau^{\prime}\right)\right]\right)\right\}$ é equivalente a $\Sigma(\tau)=$ $\left\{\left[A_{1}(\tau)\right],\left[B_{1}(\tau)\right]\right\}$. Podemos assumir que $h([0])=[0]$, substituindo $h$ com $h_{1}([z])=$ $h([z])-h([0])$ se é necessário. Então, a definição de equivalência de $\Sigma(\tau)$ e $h_{*}\left(\Sigma\left(\tau^{\prime}\right)\right)$ implica que $h_{*}\left(\left[A_{1}(\tau)\right]\right)=\left[A_{1}(\tau)\right]$ e $h_{*}\left(\left[B_{1}\left(\tau^{\prime}\right)\right]\right)=\left[B_{1}(\tau)\right]$. Tomamos um levantamento $\tilde{h}$ de $h$ com $\tilde{h}(0)=0$. Então $\tilde{h}(z)=\alpha z$ para algum $\alpha \in \mathbb{C}$. Daqui concluímos que $\tilde{h}(1)=\alpha=1$, e $\tilde{h}\left(\tau^{\prime}\right)=\alpha \tau^{\prime}=\tau$. A recíproca é obvia.

Como todo toro marcado $\left[R, \Sigma_{p}\right]$ é representado por $\left[R_{\tau}, \Sigma(\tau)\right]$ para algum $\tau \in \mathbb{H}$, Teorema 3.3 mostra que $T_{1}$ é identificado com $\mathbb{H}$.

Outro método para marcar as superfícies de Riemann é realizado através de difeomorfismos preservando orientação.

Para a segunda construção fixamos uma superfície de Riemann $R$ de gênero $g \geq 1$. Consideremos um par arbitrário $(S, f)$ de uma superfície de Riemann fechada $S$ e um difeomorfismo preservando orientação $f: R \rightarrow S$. Dois pares $(S, f)$ e $\left(S^{\prime}, g\right)$ são equivalentes se $g \circ f^{-1}: S \rightarrow S^{\prime}$ é homotópico a um biholomorfismo $h: S \rightarrow S^{\prime}$. Denotamos por $[S, f]$ a classe de equivalência de $(S, f)$.

Definição 3.4. O conjunto das classes de equivalência $[S, f]$, denotado por $T(R)$, é chamado o espaço de Teichmüller de $R$.

Observação 3.5. Um ponto $[S, f] \in T(R)$ representa uma deformação da estrutura complexa em $R$. De fato, seja $\mathcal{A}=\left\{\left(V_{\alpha}, w_{\alpha}\right)\right\}_{\alpha \in A}$ o atlas holomorfo em $S$ que define sua estrutura complexa, então o atlas $\mathcal{A}^{\prime}=\left\{\left(f^{-1}\left(V_{\alpha}\right), w_{\alpha} \circ f\right)\right\}_{\alpha \in A}$ em $R$ é holomorfo o qual define uma estrutura complexa em $R$. Seja $R_{f}$ a superfície de $\operatorname{Riemann}\left(R, \mathcal{A}^{\prime}\right)$, então temos que a aplicação identidade $I d: R \rightarrow R_{f}$ é um difeomorfismo preservando orientação e que $f: R_{f} \rightarrow S$ é um biholomorfismo. Portanto $[S, f]=\left[R_{f}, I d\right]$ em $T(R)$.

No que segue desta seção, vamos ver que $T_{g}$ e $T(R)$ são identificados.

Fixamos uma marcação $\Sigma=\left\{\left[A_{j}\right],\left[B_{j}\right]\right\}_{j=1}^{g}$ em uma superfície de Riemann fechada $R$ de gênero $g \geq 1$ com ponto base em $p_{0}$. Correspondente ao ponto $[S, f] \in T(R)$, a marcação $f_{*}(\Sigma)$ em $S$ determina um ponto $\left[S, f_{*}(\Sigma)\right] \in T_{g}$, tal ponto não depende do representante de $[S, f] \in T(R)$. Assim temos uma aplicação $\Phi_{\Sigma}: T(R) \rightarrow T_{g}$ definida por

$$
\Phi_{\Sigma}([S, f])=\left[S, f_{*}(\Sigma)\right]
$$

Vamos estudar esta aplicação no caso $g=1$. 
Teorema 3.6. Sejam $R, S$ e $S^{\prime}$ toros e $f: R \rightarrow S, g: R \rightarrow S^{\prime}$ difeomorfismos preservando orientação. Então $\left[S, f_{*}(\Sigma)\right]=\left[S^{\prime}, g_{*}(\Sigma)\right]$ em $T_{1}$ se e somente se $g \circ f^{-1}: S \rightarrow S^{\prime}$ é homotópica a um biholomorfismo $h: S \rightarrow S^{\prime}$.

Prova: Suponhamos que $\left[S, f_{*}(\Sigma)\right]=\left[S^{\prime}, g_{*}(\Sigma)\right]$. Tomamos dois pontos $\tau, \tau^{\prime} \in \mathbb{H}$ tal que $[R, \Sigma]=\left[R_{\tau}, \Sigma(\tau)\right]$ e $\left[S, f_{*}(\Sigma)\right]=\left[R_{\tau^{\prime}}, \Sigma\left(\tau^{\prime}\right)\right]$, onde $R_{\tau}, R_{\tau^{\prime}}, \Sigma(\tau)$ e $\Sigma\left(\tau^{\prime}\right)$ são definidos como no Teorema 3.3. Consideramos $f$ e $g$ como difeomorfismos de $R_{\tau}$ a $R_{\tau^{\prime}}$. Podemos assumir que seus levantamentos $\widetilde{f}$ e $\widetilde{g}$ levam 0,1 e $\tau$ a 0,1 e $\tau^{\prime}$, respectivamente. Desse modo obtemos uma homotopia entre $\tilde{f}$ e $\widetilde{g}$ definindo

$$
\widetilde{F}_{t}(z)=(1-t) \widetilde{f}(z)+t \widetilde{g}(z), \quad z \in \mathbb{C}, 0 \leq t \leq 1
$$

Pondo $F_{t}([z])=\left[\widetilde{F}_{t}(z)\right]$, obtemos uma homotopia entre $f$ e $g$. Daqui, $g \circ f^{-1}: R_{\tau^{\prime}} \rightarrow R_{\tau^{\prime}}$ é homotópica à identidade.

Reciprocamente, suponhamos que $g \circ f^{-1}: S \rightarrow S^{\prime}$ é homotópica a um biholomorfismo $h$, então as duas aplicações $h \circ f$ e $g$ de $R$ a $S^{\prime}$ são homotópicas. Seja $F_{t}: R \rightarrow S^{\prime}, 0 \leq t \leq$ 1 , a homotopia entre $h \circ f$ e $g$, e seja $p_{0}$ um ponto base para a marcação $\Sigma$. Seja $C_{0}$ a curva contínua em $S^{\prime}$ de $(h \circ f)\left(p_{0}\right)$ a $g\left(p_{0}\right)$ dado por $F_{t}\left(p_{0}\right), 0 \leq t \leq 1$. Pelo isomorfismo $T_{C_{0}}$ : $\pi_{1}\left(S^{\prime},(h \circ f)\left(p_{0}\right)\right) \rightarrow \pi_{1}\left(S^{\prime}, g\left(p_{0}\right)\right)$ induzido por $C_{0}$, vemos que as marcações $(h \circ f)_{*}(\Sigma)$ e $g_{*}(\Sigma)$ em $S^{\prime}$ são equivalentes, o que implica que $\left[S, f_{*}(\Sigma)\right]=\left[S^{\prime}, g_{*}(\Sigma)\right]$.

Observação 3.7. Para um arbitrário toro marcado $\left[S, \Sigma_{p}\right]$, encontramos um difeomorfismo preservando orientação $f: R \rightarrow S$ tal que $\left[S, \Sigma_{p}\right]=\left[S, f_{*}(\Sigma)\right]$. De fato, tomando dois pontos $\tau_{0}, \tau \in \mathbb{H}$ tal que $[R, \Sigma]=\left[R_{\tau_{0}}, \Sigma\left(\tau_{0}\right)\right]$ e $\left[S, \Sigma_{p}\right]=\left[R_{\tau}, \Sigma(\tau)\right]$ vemos que a aplicação linear

$$
\widetilde{f}_{\tau}(z)=\frac{\left(\tau-\bar{\tau}_{0}\right) z-\left(\tau-\tau_{0}\right) \bar{z}}{\tau-\bar{\tau}_{0}}, \quad z \in \mathbb{C}
$$

induz um difeomorfismo preservando orientação $f_{\tau}: R_{\tau_{0}} \rightarrow R_{\tau}$ pondo $f_{\tau}([z])=\left[\tilde{f}_{\tau}(z)\right]$. Em particular, obtemos $\left(f_{\tau}\right)_{*}\left(\Sigma\left(\tau_{0}\right)\right)=\Sigma(\tau)$.

Assim, do Teorema 3.6 e a Observação 3.7 , obtemos que aplicação $\Phi_{\Sigma}$ definida em (3.1) é uma bijeção, no caso de gênero 1 , e portanto temos a identificação entre $T_{1}$ e $T(R)$.

Mais geralmente, o seguinte Teorema garante que a afirmação de acima se cumpre para qualquer gênero $g \geq 2$.

Teorema 3.8. Ver [7, pág.14]. A aplicação $\Phi_{\Sigma}: T(R) \rightarrow T_{g}$ definida em (3.1) é bijetora.

Definição 3.9. Seja $R$ uma superfície de Riemann de gênero $g \geq 1$. O conjunto $\operatorname{Mod}(R)$ de todas as classes de homotopia $[w]$ de difeomorfismos preservando orientação $w: R \rightarrow R$ é chamado o grupo modular de Teichmüller. 
Tem-se uma ação de $\operatorname{Mod}(R)$ sobre $T(R)$ dado por:

$$
[w]_{*}([S, f])=\left[S, f \circ w^{-1}\right]
$$

para qualquer $[S, f] \in T(R)$. Chamamos a todo $[w]_{*}$ uma transformação modular de Teichmüller.

Desde que para toda superfície de Riemann fechada $S$ de gênero $g$ existe um difeomorfismo preservando orientação de $R$ sobre $S$ (Ver 1.20), o espaço moduli $\mathcal{M}_{g}$ é identificado com o espaço quociente $T(R) / \operatorname{Mod}(R)$. Consequentemente podemos estudar o espaço $\mathcal{M}_{g}$ através de $T(R)$ e $\operatorname{Mod}(R)$.

\subsection{Coordenadas de Fricke}

Na seção anterior identificamos o espaço $T_{1}$, nesta seção o objetivo é identificar o espaço $T_{g}, g \geq 2$. Para isso, utilizaremos alguns resultados dos capítulos anteriores.

Seja $R$ uma superfície de Riemann fechada de gênero $g \geq 2$. Para um modelo Fuchsiano $\Gamma$ de $R, R=\mathbb{H} / \Gamma$, lembrar que temos um isomorfismo entre $\pi_{1}(R, p)$ e $\Gamma$ (Teorema 2.20), denotamos por $\alpha_{j}$ e $\beta_{j}$ os elementos de $\Gamma$ correspondentes aos elementos $\left[A_{j}\right]$ e $\left[B_{j}\right]$ de $\pi_{1}(R, p)$ para todo $j=1, \ldots, g$. Sabemos que se $\Gamma$ é um modelo Fuchsiano, então para qualquer $\delta \in P S L(2, \mathbb{R})$, o grupo $\Gamma^{\prime}=\delta \Gamma \delta^{-1}$ é também um modelo Fuchsiano de $R$ (Teorema 2.28).

Para atribuir únicamente um modelo Fuchsiano $\Gamma$ a uma marcação $\Sigma$ dada em $R$, impomos as seguintes condições de normalização:

(i) $\beta_{g}$ tem seus pontos fixos repulsor e atractor em 0 e $\infty$ respectivamente.

(ii) $\alpha_{g}$ tem seu ponto fixo atractor em 1 .

Observação 3.10. Dado uma marcação $\Sigma$ em uma superfície de Riemann fechada $R$ de gênero $g \geq 2$, sempre existe um modelo Fuchsiano de $R$ a qual satifaz as condições de normalização. De fato, sabemos que $\alpha_{g}$ e $\beta_{g}$ são hiperbólicos, como não comutam, pelo Lema 1.61 temos que Fix $\left(\alpha_{g}\right) \cap \operatorname{Fix}\left(\beta_{g}\right)=\emptyset$. Logo, conjugando $\Gamma$ em $P S L(2, \mathbb{R})$, se fosse necessário, $\alpha_{g}$ e $\beta_{g}$ satisfazem as condições de normalização (i) e (ii)

Proposição 3.11. Para uma marcação $\Sigma$ dada em uma superfície de Riemann fechada de gênero $g \geq 2$, um sistema canônico de geradores $\left\{\alpha_{j}, \beta_{j}\right\}_{j=1}^{g}$ de um modelo Fuchsiano $\Gamma$ de $R$ que satifaz (i) e (ii) respeito a $\Sigma$ é únicamente determinado pelo ponto $[R, \Sigma]$ em $T_{g}$. 
Prova: Seja $R^{\prime}$ e $\Sigma^{\prime}$ tal que $\left[R^{\prime}, \Sigma^{\prime}\right]=[R, \Sigma]$ em $T_{g}$, logo existe $f: R \rightarrow R^{\prime}$ biholomorfismo tal que $f_{*}(\Sigma)$ é equivalente a $\Sigma^{\prime}$. Um levantamento $\tilde{f}$ de $f$ em $\mathbb{H}, \widetilde{f} \in A u t(\mathbb{H})$, é tomado tal que satisfaz

$$
\alpha_{j}^{\prime}=\tilde{f} \circ \alpha_{j} \circ \widetilde{f}^{-1} \text { e } \beta_{j}^{\prime}=\tilde{f} \circ \beta_{j} \circ \widetilde{f}^{-1}
$$

onde $\left\{\alpha_{j}^{\prime}, \beta_{j}^{\prime}\right\}_{j=1}^{g}$ é o sistema de geradores de um modelo Fuchsiano de $R^{\prime}$ que satisfaz (i) e (ii) respeito a $\Sigma^{\prime}$. Da condição (i), temos que $\widetilde{f}(z)=\lambda z$ para algum $\lambda>0$. Além disso, por (ii), $\alpha_{g}$ e $\alpha_{g}^{\prime}$ têm um ponto fixo comum em 1 , daí $\lambda=1$, assim $\widetilde{f}=$ Id. Portanto $\alpha_{j}=\alpha_{j}^{\prime}$ e $\beta_{j}=\beta_{j}^{\prime}$.

Chamamos a esse grupo Fuchsiano $\Gamma$ o modelo Fuchsiano normalizado de $[R, \Sigma]$. O sistema de geradores $\left\{\alpha_{j}, \beta_{j}\right\}_{j=1}^{g}$ é referido como seu sistema canônico de geradores, o qual satifaz

$$
\prod_{j=1}^{g}\left[\alpha_{j}, \beta_{j}\right]=\mathrm{Id}, \text { onde }\left[\alpha_{j}, \beta_{j}\right]=\alpha_{j} \circ \beta_{j} \circ \alpha_{j}^{-1} \circ \beta_{j}^{-1}
$$

Lema 3.12. Seja $\left\{\alpha_{j}, \beta_{j}\right\}_{j=1}^{g}$ um sistema canônico de geradores do modelo Fuchsiano normalizado $\Gamma$ para um ponto $[R, \Sigma]$ em $T_{g}$. Se um elemento $\gamma(z)=\frac{a z+b}{c z+d} d e\left\{\alpha_{j}, \beta_{j}\right\}_{j=1}^{g}$ não coincide $\operatorname{com} \beta_{g}$, então $b c \neq 0$.

Prova: No caso onde $b=c=0$, temos $\operatorname{Fix}(\gamma)=\operatorname{Fix}\left(\beta_{g}\right)=\{0, \infty\}$, e então $\gamma$ e $\beta_{g}$ seriam comutativos, contradição. Logo, no caso onde $b=0$ e $c \neq 0$, obtemos $\operatorname{Fix}(\gamma)=$ $\operatorname{Fix}\left(\beta_{g}\right)=\{0\}$. Mas, $\gamma$ e $\beta_{g}$ são não comutativos, isto contradiz o fato que $\Gamma$ é Fuchsiano. Analogamente, o caso onde $b \neq 0$ e $c=0$, obtemos uma contradição.

Pelo lema anterior, o sistema canônico de geradores $\left\{\alpha_{j}, \beta_{j}\right\}_{j=1}^{g}$ do modelo Fuchsiano normalizado $\Gamma$ para um ponto $[R, \Sigma]$ em $T_{g}$ é escrito únicamente na forma

$$
\begin{gathered}
\alpha_{j}=\frac{a_{j} z+b_{j}}{c_{j} z+d_{j}}, \quad a_{j}, b_{j}, c_{j} \in \mathbb{R}, \quad c_{j}>0, \quad a_{j} d_{j}-b_{j} d_{j}=1 \\
\beta_{j}=\frac{a_{j}^{\prime} z+b_{j}^{\prime}}{c_{j}^{\prime} z+d_{j}^{\prime}}, \quad a_{j}^{\prime}, b_{j}^{\prime}, c_{j}^{\prime} \in \mathbb{R}, \quad c_{j}^{\prime}>0, \quad a_{j}^{\prime} d_{j}^{\prime}-b_{j}^{\prime} d_{j}^{\prime}=1
\end{gathered}
$$

para cada $j=1,2, \ldots, g-1$.

Agora, definimos as coordenadas de Fricke $\mathcal{F}_{g}: T_{g} \rightarrow \mathbb{R}^{6 g-6}$ por

$$
\mathcal{F}_{g}([R, \Sigma])=\left(a_{1}, c_{1}, d_{1}, a_{1}^{\prime}, c_{1}^{\prime}, d_{1}^{\prime}, \ldots, a_{g-1}, c_{g-1}, d_{g-1}, a_{g-1}^{\prime}, c_{g-1}^{\prime}, d_{g-1}^{\prime}\right)
$$

A imagem $F_{g}=\mathcal{F}_{g}\left(T_{g}\right)$ é chamado o espaço de Fricke das superfícies de Riemann 
fechadas de gênero $g$. A topología de $F_{g}$ é induzida por $\mathbb{R}^{6 g-6}$.

Teorema 3.13. A aplicação $\mathcal{F}_{g}: T_{g} \rightarrow \mathbb{R}^{6 g-6}$ definida em (3.3) é injetora.

Prova: Precisamos mostrar que todo ponto $\mathcal{F}_{g}([R, \Sigma])=\left(a_{1}, c_{1}, d_{1}, \ldots, a_{g-1}^{\prime}, c_{g-1}^{\prime}, d_{g-1}^{\prime}\right)$ em $F_{g}$ determina únicamente o sistema canônico de geradores $\left\{\alpha_{j}, \beta_{j}\right\}_{j=1}^{g}$ do modelo Fuchsiano $\Gamma$ para o ponto $[R, \Sigma]$ em $T_{g}$.

Para cada $j(j=1,2, \ldots, g-1), b_{j}$ é obtido da relação $a_{j} d_{j}-b_{j} c_{j}=1 \operatorname{com} c_{j}>0$, e daí $\alpha_{j}$ é determinado únicamente por $\mathcal{F}_{g}([R, \Sigma])$. Analogamente, cada $\beta_{j}(j=1,2, \ldots, g-1)$ também é determinado.

Resta provar que $\alpha_{g}$ e $\beta_{g}$ são determinados por $\mathcal{F}_{g}([R, \Sigma])$. Pela condição de normalização (i) para $\Gamma$, temos que $\beta_{g}(z)=\lambda z \operatorname{com} \lambda>1$. Pela condição (ii), $\alpha_{g}$ tem seu ponto fixo atractor em 1 , e assim

$$
a_{g}+b_{g}=c_{g}+d_{g}
$$

Se $\gamma=\prod_{j=1}^{g-1}\left[\alpha_{j}, \beta_{j}\right]$, da fundamental relação $\prod_{j=1}^{g}\left[\alpha_{j}, \beta_{j}\right]=\mathrm{Id}$, temos que $\gamma \circ \alpha_{g}=$ $\beta_{g} \circ \alpha_{g} \circ \beta_{g}^{-1}$. Pondo

$$
\gamma(z)=\frac{a z+b}{c z+d}, \quad a, b, c, d \in \mathbb{R} a d-b c=1
$$

Temos as seguintes equações:

$$
\begin{array}{r}
(a-1) a_{g}+b c_{g}=0 \\
c a_{g}+\left(d-\lambda^{-1}\right) c_{g}=0 \\
c b_{g}+(d-1) d_{g}=0
\end{array}
$$

Desde que ou $a_{g}$ ou $c_{g}$ não é zero, das duas primeiras equações de acima, temos que $a-1=\lambda(1-d)$. Se $a=1$, então $d=1$, e daí $\operatorname{tr}^{2}(\gamma)=4$, o que implica que $\gamma$ é parabólico, absurdo. Assim, segue-se que $a \neq 1, d \neq 1$, e $\lambda=(a-1) /(1-d)$. Portanto temos determinado $\beta_{g}$.

Temos também

$$
\begin{aligned}
& a_{g}=\frac{b c_{g}}{1-a} \\
& d_{g}=\frac{c b_{g}}{1-d}
\end{aligned}
$$

Destas equações e a relação (3.4) temos

$$
\frac{a+b-1}{1-a} c_{g}=\frac{c+d-1}{1-d} b_{g}
$$


Se $c+d=1$, então $a+b=1$, pois $c_{g} \neq 0$. Assim, da relação $a d-b c=1$, encontramos $a+$ $d=2$, e daí $\gamma$ é parabólico, contradição. Portanto $c+d \neq 1$ e assim temos determinado $\alpha_{g}$ por $\mathcal{F}_{g}([R, \Sigma])$

\subsection{Estrutura conforme}

Seja $\left(M, d s^{2}\right)$ uma variedade Riemanniana orientada 2-dimensional (superfície Riemanniana). A métrica Riemanniana pode ser representada como

$$
d s^{2}=E d x^{2}+2 F d x d y+G d y^{2}
$$

em termos de uma carta local $(U,(x, y))$ de $M$. Deixando $z=x+i y$, a equação (3.5) pode-se escrever unicamente na forma

$$
d s^{2}=\lambda|d z+\mu d \bar{z}|^{2}
$$

com $d z=d x+i d y$ e $d \bar{z}=d x-i d y$ onde $\lambda$ é uma função positiva em $U$ e $\mu$ é uma função suave com valores complexos com $|\mu|<1$ definida em $U$. De fato, $\lambda$ e $\mu$ são dados por

$$
\begin{gathered}
\lambda=\frac{1}{4}\left(E+G+2 \sqrt{E G-F^{2}}\right) \\
\mu=\frac{E-G+2 i F}{E+G+2 \sqrt{E G-F^{2}}}
\end{gathered}
$$

Nestas condições temos a seguinte definição.

Definição 3.14. Seja $(U,(u, v))$ uma carta local de $M$. Dizemos que as coordenadas locais $(u, v)$ em $U$ são coordenadas isotérmicas para $d s^{2}$ se $d s^{2}$ é representado como

$$
d s^{2}=\rho\left(d u^{2}+d v^{2}\right)
$$

em $U$, onde $\rho$ é uma função suave positiva em $U$.

Aqui, nos assumimos que ambas orientações induzidas pelas coordenadas $(x, y) \mathrm{e}$ $(u, v)$ em $U$ coincidem com a orientação dada em $M$.

A coordenada complexa $w=u+i v$ é também chamada coordenada isotérmica para $d s^{2}$.

Temos que uma coordenada isotérmica $w$ para $d s^{2}$ satisfaz:

$$
d s^{2}=\rho|d w|^{2}=\rho\left|w_{z} d z+w_{\bar{z}} d \bar{z}\right|^{2}=\rho\left|w_{z}\right|^{2}\left|d z+\frac{w_{\bar{z}}}{w_{z}} d \bar{z}\right|^{2}
$$


onde

$$
w_{\bar{z}}=\frac{1}{2}\left(w_{x}+i w_{y}\right), \quad w_{z}=\frac{1}{2}\left(w_{x}-i w_{y}\right)
$$

Logo, comparando com a equação (3.6), concluímos que uma coordenada isotérmica $w$ para $d s^{2}$ existe se a equação diferencial parcial

$$
\frac{\partial w}{\partial \bar{z}}=\mu \frac{\partial w}{\partial z}
$$

tem uma solução suave $w$. Esta equação é chamada a equação de Beltrami.

Nestas condições, o seguinte resultado pode-se ver em [9, pág.155].

Teorema 3.15. A equação de Beltrami (3.8) tem soluções suaves.

Como consequência deste teorema temos o seguinte corolário.

Corolário 3.16. Seja $\left(M, d s^{2}\right)$ é uma superfície Riemanniana, então para cada ponto $p \in M$, existe uma carta local $(U, w)$ em $p$ tal que $w=u+i v$ é uma coordenada isotérmica para $d s^{2}$.

Observação 3.17. Sejam $f: U \rightarrow V$ e $g: V \rightarrow W$, com $U, V$ e $W$ abertos de $\mathbb{C}$, duas funções suaves. Da regra da cadeia, temos

$$
\begin{aligned}
& (g \circ f)_{z}=\left(g_{\zeta} \circ f\right) f_{z}+\left(g_{\bar{\zeta}} \circ f\right) \bar{f}_{z} \\
& (g \circ f)_{\bar{z}}=\left(g_{\zeta} \circ f\right) f_{\bar{z}}+\left(g_{\bar{\zeta}} \circ f\right) \bar{f}_{\bar{z}}
\end{aligned}
$$

$\operatorname{com} \zeta=f(z)$. Resolvendo esta equação obtemos

$$
\begin{aligned}
g_{\zeta} \circ f & =\frac{1}{J}\left[(g \circ f)_{z} \bar{f}_{\bar{z}}-(g \circ f)_{\bar{z}} \bar{f}_{z}\right] \\
g_{\bar{\zeta}} \circ f & =\frac{1}{J}\left[(g \circ f)_{\bar{z}} f_{z}-(g \circ f)_{z} f_{\bar{z}}\right]
\end{aligned}
$$

onde $J=\left|f_{z}\right|^{2}-\left|f_{\bar{z}}\right|^{2}=\frac{\partial(u, v)}{\partial(x, y)}=\frac{\partial(w, \bar{w})}{\partial(z, \bar{z})}$ se $w=u+i v=f(z), z=x+i y$.

Em particular, fazendo $g=f^{-1}$ em (3.10) temos

$$
\left(f^{-1}\right)_{\zeta} \circ f=\bar{f}_{\bar{z}} / J \quad\left(f^{-1}\right)_{\bar{\zeta}} \circ f=-f_{\bar{z}} / J
$$

Teorema 3.18. Seja $\left(M, d s^{2}\right)$ uma superfície Riemanniana. Então $M$ possui uma estrutura complexa induzida por $d s^{2}$, isto é, $M$ resulta uma superfície de Riemann.

Prova: Pelo Corolário 3.16, existe um atlas $\mathcal{A}=\left\{U_{i}, w_{i}\right\}_{i \in I}$ de $M$ tal que cada $w_{i}$ é uma coordenada isotérmica para $d s^{2}$.

Afirmação: $\mathrm{O}$ atlas $\mathcal{A}$ é holomorfo. 
De fato, sejam $\eta=w_{i}, w=w_{j} \in \mathcal{A}$ tal que $U_{i} \cap U_{j} \neq \emptyset$. Como $\eta$ e $w$ satisfazem a equação de Beltrami, temos que

$$
\begin{aligned}
\eta_{\bar{w}} & =\eta_{z} z_{\bar{w}}+\eta_{\bar{z}} \bar{z}_{\bar{w}} \\
& =\frac{1}{\left|w_{z}\right|^{2}-\left|w_{\bar{z}}\right|^{2}}\left(-\eta_{z} w_{\bar{z}}+\eta_{\bar{z}} w_{z}\right)=0 .
\end{aligned}
$$

Definição 3.19. Seja $M$ uma variedade suave.

(i) Duas métricas Riemannianas $g_{1}$ e $g_{2}$ em $M$ são equivalentemente conformes, denotado por $g_{1} \sim g_{2}$, se existe uma função suave $\varphi$ tal que $g_{1}=e^{\varphi} g_{2}$.

(ii) Uma classe de equivalência sob é chamado uma estrutura conforme em $M$.

(iii) Um difeomorfismo preservando orientação $f:\left(M, g_{1}\right) \rightarrow\left(N, g_{2}\right)$ entre duas variedades Riemannianas é uma aplicação conforme se a métrica induzida por $g_{2}$ sob $f$ em $M$ é equivalentemente conforme a $g_{1}$. Neste caso dizemos que $\left(M, g_{1}\right) e\left(N, g_{2}\right)$ são equivalentemente conformes ou têm a mesma estrutura conforme.

Sejam $g_{1}$ e $g_{2}$ duas métricas Riemannianas na superfície suave $M$ tal que $g_{1} \sim g_{2}$, então as métricas $g_{1}$ e $g_{2}$ em $M$ definem a mesma estrutura complexa em $M$. De fato, se $(U, z)$ é uma carta local em $M$, temos que $g_{1}=\lambda_{1}\left|d z+\mu_{1} d \bar{z}\right|^{2}$ e $g_{2}=\lambda_{2}\left|d z+\mu_{2} d \bar{z}\right|^{2}$ para alguns $\lambda_{i}, \mu_{i}, i=1,2$ como em (3.6). Do Teorema 3.18, sabemos que as soluções suaves de

$$
\frac{\partial w}{\partial \bar{z}}=\mu_{1} \frac{\partial w}{\partial z} \quad \frac{\partial w}{\partial \bar{z}}=\mu_{2} \frac{\partial w}{\partial z}
$$

definem a estrutura complexa $\mathcal{A}_{1}$ e $\mathcal{A}_{2}$ em $M$ induzida por $g_{1}$ e $g_{2}$, respectivamente. Mas, como $g_{1} \sim g_{2}$, temos que $\lambda_{1}=e^{\varphi} \lambda_{2}$ e $\mu_{1}=\mu_{2}$. Portanto, pelo Teorema 3.18, $\mathcal{A}_{1}=\mathcal{A}_{2}$.

Reciprocamente, se $M$ tem uma estrutura complexa então existe uma estrutura conforme em $M$. De fato, sendo $M$ uma superfície de Riemann, dependendo de se seu recobrimento universal $\widetilde{M}$ é biholomorfo à $\widehat{\mathbb{C}}, \mathbb{C}$ ou $\mathbb{H}$ tomamos a métrica Riemanniana em $M$ a qual é induzida pela

$$
\begin{aligned}
\text { Métrica esférica } & =\frac{4}{\left(1+|z|^{2}\right)^{2}}|d z|^{2} . \\
\text { Métrica Euclideana } & =|d z|^{2} . \\
\text { Métrica de Poincaré } & =\frac{|d z|^{2}}{(\text { Imz })^{2}} .
\end{aligned}
$$

respectivamente. 
Assim temos a seguinte proposição.

Proposição 3.20. Seja $M$ uma superfície suave. Então existe uma correspondência biunívoca entre as estruturas conformes em $M$ e as estruturas complexas em $M$.

Observação 3.21. Esta proposição mostra que, no caso de dimensão 2, os conceitos de estrutura complexa e estrutura conforme são equivalentes, porém em dimensões maiores isto não é certo. 


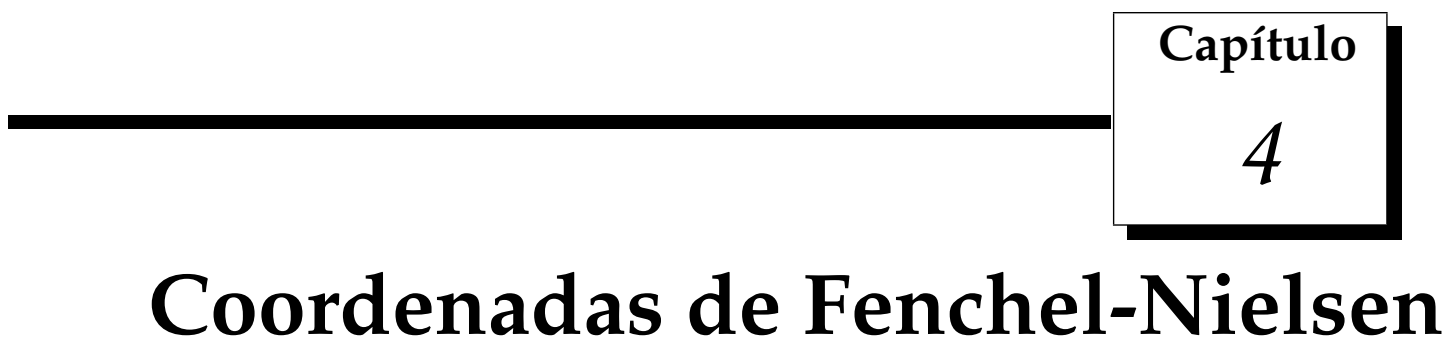

Neste Capítulo, apresentamos as coordenadas de Fenchel-Nielsen o qual identifica o espaço $T_{g}, g \geq 2$ com o espaço Euclideano $\mathbb{R}^{6 g-6}$. Para isto, utilizando utilizando o conceito de superfície hiperbólica, apresentamos uma definição equivalente de $T_{g}$. A principal referência deste capítulo é [3]. Outra excelente referência é [7].

\subsection{Estruturas hiperbólicas: Definições e resultados}

Neste seção começamos com algumas observações respeito de estruturas hiperbólicas e estruturas complexas.

Definição 4.1. Seja $S$ uma superfície sem fronteira. Um atlas $\mathcal{A}$ é chamado hiperbólico se satisfaz as seguintes propiedades:

(i) $\varphi(U) \subset \mathbb{H}$, para todo $(U, \varphi) \in \mathcal{A}$.

(ii) Se $(U, \varphi) \in \mathcal{A} e\left(U^{\prime}, \varphi^{\prime}\right) \in \mathcal{A}$, então para cada componente conexa $V$ de $U \cap U^{\prime}$ existe um $m \in \operatorname{Isom}^{+}(\mathbb{H})$ tal que $\varphi^{\prime} \circ \varphi^{-1}$ coincide com $m$ em $\varphi(V)$.

Seja $\delta>0$ e $0<\theta<2 \pi$. Um subconjunto $V$ de $\mathbb{H}$ é chamado um sector circular de raio $\delta$ e ângulo $\theta$ em $p_{0} \in \mathbb{H}$ se ele tem a seguinte forma, onde $(\eta, \sigma)$ são coordenadas polares baseadas em $p_{0}$.

$$
V=\{(\eta, \sigma) \in \mathbb{H} \mid 0<\eta<\delta, 0 \leq \sigma \leq \theta\} \cup\left\{p_{0}\right\}
$$

Um semi-disco é um sector circular de ângulo $\pi$.

Seja $S$ uma superfície com fronteira suave por partes $\partial S$ não vazia. O conjunto int $S:=$ $S-\partial S$ é chamado o interior de $S$. A fronteira $\partial S$ é composta por arcos suaves, os 
lados de $S$. Um lado pode ter 0,1 ou 2 pontos extremos. Uma curva fechada suave da fronteira é um lado sem pontos extremos. Todo ponto extremo de um lado é o ponto extremo de um lado adjacente e é chamado um vértice de $S$. Um ponto de $\partial S$ o qual não é um vértice é chamado ponto ordinário da fronteira.

Definição 4.2. Seja $S$ uma superfície com fronteira não vazia. Um atlas $\mathcal{A}$ de $S$ é chamado hiperbólico se satisfaz as seguintes propriedades:

(i) Para cada $p \in S$ existe um sistema coordenado $(U, \varphi) \in \mathcal{A}$ com $p \in U$ tal que $\varphi(U) \subset \mathbb{H}$ é Um sector circular de ângulo $\theta \leq \pi$ em $\varphi(p)$, se pé um vértice.

Um semi-disco em $\varphi(p)$, se pé um ponto ordinário da fronteira.

$U m$ disco aberto com centro em $\varphi(p)$, se pé um ponto interior.

(ii) $\operatorname{Se}(U, \varphi) \in \mathcal{A} e\left(U^{\prime}, \varphi^{\prime}\right) \in \mathcal{A}$, então para cada componente conexa $V$ de $U \cap U^{\prime}$ existe uma $m \in \operatorname{Isom}^{+}(\mathbb{H})$ tal que $\varphi^{\prime} \circ \varphi^{-1}$ coincide com $m$ em $\varphi(V)$.

Definição 4.3. Um atlas hiperbólico maximal em S é chamado uma estrutura hiperbólica. A estrutura hiperbólica é completa se o espaço métrico $S$ (com a métrica induzida pela estrutura hiperbólica) é completa. Uma superfície conexa junto com uma estrutura hiperbólica completa é chamada uma superfície hiperbólica.

Sejam $S_{1}, \ldots, S_{m}$ superfícies hiperbólicas disjuntas e sejam $\gamma_{1}, \gamma_{1}^{\prime}, \gamma_{2}, \gamma_{2}^{\prime} \ldots, \gamma_{n}, \gamma_{n}^{\prime}$ distintos lados disjuntos de $S:=S_{1} \cup \ldots \cup S_{m}$. Suponhamos que para cada $k$, os lados são parametrizados na forma $\gamma_{k}, \gamma_{k}^{\prime}: I_{k} \rightarrow S$ com a mesma velocidade constante, $I_{k}$ é um intervalo. Quando $\gamma_{k}$ e $\gamma_{k}^{\prime}$ são geodésicas fechadas suaves, então consideramos $I_{k}=\mathbb{R} /\left[t \mapsto t+a_{k}\right]$ para algum $a_{k}$. Definimos uma relação de equivalência em $S$ pela condiçaõ de colagem

$$
\gamma_{k}(t)=\gamma_{k}^{\prime}(t), \quad t \in I_{k}, \quad k=1, \ldots, n
$$

Definição 4.4. Sob as hipóteses acima, $F=S_{1}+\ldots+S_{m}$ mod (4.1), é o espaço quociente da união disjunta $S=S_{1} \cup \ldots \cup S_{m}$ com respeito à relação de equivalência definida em (4.1).

Exemplo 4.5. Colagem de geodésicas fechadas. Sejam $S, S^{\prime}$ superfícies hiperbólicas (não necessariamente distintas) e suponhamos que $\gamma$ em $S$ e $\gamma^{\prime}$ em $S^{\prime}$ são geodésicas da fronteira com o mesmo comprimento, por exemplo $l$. Parametrizamos $\gamma$ e $\gamma^{\prime}$ periodicamente em $\mathbb{R}$ com período 1 e velocidade $l$. Se $S$ coincide com $S^{\prime}$, então assumimos que $\gamma$ e $\gamma^{\prime}$ são distintos e têm a mesma orientação na fronteira, isto é, $S$ e $S^{\prime}$ estão ambos no lado esquerdo ou ambos estão no lado direito de $\gamma$ e $\gamma^{\prime}$. Seja

$$
F=S+S^{\prime} \bmod \left(\gamma(t)=\gamma^{\prime}(-t), t \in \mathbb{R}\right)
$$


As duas geodésicas $\gamma$ e $\gamma^{\prime}$ projectam-se a uma geodésica fechada simples $\gamma_{1}$ de comprimento $l$ em $F$. A condição de colagem em (4.2) pode ser substituída por

$$
\gamma(t)=\gamma^{\prime}(\alpha-t), \quad t \in \mathbb{R}
$$

com um arbitrário parâmetro de torção $\alpha \in \mathbb{R}$.

O seguinte teorema nos garante, sob algumas condições, que o colagem de superfícies hiperbólicas é uma superfície hiperbólica.

Teorema 4.6. Ver [3, pág.13]. Com a notação da Definição 4.4, um ciclo de vértices é o conjunto de todos os vértices de $S_{1}, \ldots, S_{m}$ tal que juntos definem um ponto interior ou um ponto da fronteira de $F$.

Assumamos que se satifazem:

(i) Para cada ciclo de vértices o qual da um ponto interior de F a soma dos ângulos interiores nos vértices é $2 \pi$.

(ii) Para cada ciclo de vértices o qual da um ponto da fronteira de F a soma dos ângulos interiores nos vértices é $\leq \pi$.

Então F possui uma única estrutura hiperbólica tal que a projeção natural $\sigma: S_{1} \cup \ldots \cup S_{m} \rightarrow F$ é uma isometria local.

Além disso, se temos que a seguinte condição é satisfeita

(iii) F é conexo, as estruturas hiperbólicas de $S_{1}, \ldots, S_{m}$ são completos, e para qualquer par de lados não adjacentes na lista $\gamma_{1}, \ldots, \gamma_{n}^{\prime}$ estando na mesma superfície $S_{\mu}$ tem distância positiva. Então a estrutura hiperbólica de F é completa.

No caso de superfícies fechadas de gênero $g \geq 2$, o conceito de superfície hiperbólica é equivalente ao conceito de estrutura complexa.

Proposição 4.7. Seja $R$ uma superfície fechada de gênero $g \geq 2$. Então, existe uma correspondência biunívoca entre as estruturas complexas e estruturas hiperbólicas em $R$.

Prova: Seja $R$ uma superfície fechada de gênero $g \geq 2$ com uma estrutura complexa dada, isto é, $R$ é uma superfície de Riemann. Pelo Teorema de uniformização, existe um recobrimento universal $\pi: \mathbb{H} \rightarrow R$. Sabemos que o grupo das transformações do recobrimento de $\pi$ é um subgrupo de $P S L(2, \mathbb{R})$. Daqui, tomando as inversas locais de $\pi$ como cartas locais, obtemos um atlas hiperbólico em $R$ e assim $R$ é uma superfície hiperbólica.

Reciprocamente, se $R$ é uma superfície hiperbólica então $R$ também tem estrutura complexa. Com efeito, as mudanças de coordenadas do atlas hiperbólico de $R$ são elementos de $\operatorname{PSL}(2, \mathbb{R})$ os quais são biholomorfismos de $\mathbb{H}$. Portanto, o atlas hiperbólico de 
$R$ induz naturalmente uma estrutura complexa em $R$ e assim $R$ é uma superfície de Riemann.

A Proposição 4.7 nos permite dar as seguintes definições

Definição 4.8. Uma superfície hiperbólica compacta sem fronteira é chamada uma superfície de Riemann compacta.

Diz-se que uma superfície topológica com bordo $S$ tem assinatura $(g, n)$, se $S$ é obtida de uma superfície fechada de gênero $g$ cortando-se o interior de $n$ disjuntos discos topológicos fechados. Se $n=0$ então, por definição, $S$ é uma superfíce compacta de gênero $g$.

Definição 4.9. Uma superfície hiperbólica compacta de gênero $(g, n)$ é chamada superfície de Riemann de assinatura $(g, n)$, se todas as componentes da fronteira são geodésicas fechadas suaves.

Teorema 4.10. Ver [3, pág.18]. Seja S uma superfície hiperbólica e seja c : $[a, b] \rightarrow S$ uma curva com $c(a) \in A$ e $c(b) \in B$, onde $A$ e $B$ são duas geodésicas fechadas disjuntas da fronteira de $S$. Na classe de homotopia de c com pontos extremos estando em A e B existe uma única geodésica $\gamma$. Em seus pontos extremos, $\gamma$ intersecta $A$ e $B$ perpendicularmente. Todos os outros pontos de $\gamma$ estão no interior de $S$.

Teorema 4.11. Ver [3, pág.19]. Seja $S$ uma superfície hiperbólica, sejam $A, B \subset S$ subconjuntos admissíveis ${ }^{1}$ e $c:[a, b] \rightarrow S$ com $c(a) \in A$ e $c(b) \in B$ uma curva de $A$ a $B$ (A e B não necessariamente disjuntos). Então

(i) Na classe de homotopia de c com pontos extremos estando em A e B existe uma curva de menor comprimento $\gamma$. Esta curva é um arco geodésico.

(ii) Se $\gamma$ não esta contido em $\partial S$, então $\gamma$ intersecta $\partial S$ no máximo em seus pontos extremos.

(iii) Se $A=\{p\}, p \in S$ e se $B$ é um dos seguintes casos:

Uma geodésica fechada suave da fronteira.

Um lado de $S$ o qual intersecta seus lados adjacentes sob um ângulo $\leq \pi / 2$.

Então $\gamma$ ou é um ponto ou é um arco geodésico de p a $B$ que intersecta B perpendicularmente. No ultimo caso y é a única geodésica perpendicular de p a $B$ na classe de homotopia de $c$.

(iv) Se A e B são auqisquer dos dois casos em (iii) (mas não necessariamente do mesmo), e se $\gamma$ não é um ponto, então $\gamma$ é a única perpendicular comum de A a B na classe de homotopia de c.

(v) Se c no caso (iv) é simples e $\gamma$ não é um ponto, então $\gamma$ é simples.

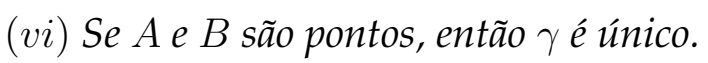

\footnotetext{
${ }^{1}$ Um subconjunto fechado e conexo $A$ de uma superfície hiperbólica $S$ é admissível se ou $A$ é um conjunto unitário ou se $A$ é um subconjunto compacto e conexo de $\partial S$.
} 


\subsection{1 $Y$-peças e $X$-peças}

Definição 4.12. Uma superfície de Riemann compacta de assinatura $(0,3)$ é chamado uma $Y$-peça ou uma calça.

Seja $G$ um hexágono hiperbólico reto com lados consecutivos $\alpha_{1}, c_{3}, \alpha_{2}, c_{1}, \alpha_{3}, c_{2}$ e seja $G^{\prime}$ uma cópia de $G$ com correspondentes lados $\alpha_{1}^{\prime}, c_{3}^{\prime}, \alpha_{2}^{\prime}, c_{1}^{\prime}, \alpha_{3}^{\prime}, c_{2}^{\prime}$. Parametrizamos os lados no intervalo $[0,1]$ com velocidade constante,

$$
\begin{aligned}
t & \mapsto \alpha_{i}(t), \quad t \mapsto \alpha_{i}^{\prime}(t) \\
t & \mapsto c_{i}(t), \quad t \quad t \in c_{i}^{\prime}(t)
\end{aligned} \quad t \in[0,1] \quad i=1,2,3 .
$$

e tal que os lados de $G$ e $G^{\prime}$ juntos formam uma curva fechada da fronteira (Ver Figura 4.1). A condição de colagem

$$
\alpha_{i}(t)=\alpha_{i}^{\prime}(t):=a_{i}(t) \quad t \in[0,1] \quad i=1,2,3 .
$$

define uma calça $Y=G+G^{\prime} \bmod (4.5)$, pois as curvas fronteias

$$
t \mapsto \gamma_{i}(t):=\left\{\begin{array}{ll}
c_{i}(2 t) & \text { se } 0 \leq t \leq 1 / 2 \\
c_{i}^{\prime}(2-2 t) & \text { se } 1 / 2 \leq t \leq 1
\end{array} \quad i=1,2,3\right.
$$

são geodésicas fechadas (os ângulos dos hexágonos são retos)

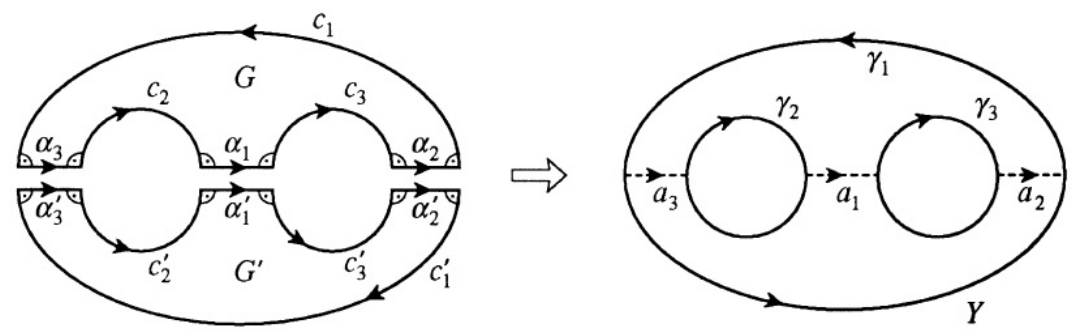

Figura 4.1: Colagem de dois hexágonos hiperbólicos retos.

A equação (4.6) também será interpretada como uma parametrização de $\gamma_{i}$ em $\mathcal{S}^{1}=\mathbb{R} /[t \mapsto t+1]$ em vez de $[0,1]$. Com qualquer interpretação (4.6) é chamado a parametrização básica das fronteiras e $Y$ é dito dado na forma básica.

Proposição 4.13. Seja S uma $Y$-peça arbitrária. Para todo par de geodésicas da fronteira de $S$ existe um único arco geodésico simples e perpendicular às geodésicas. As três perpendiculares juntas decompõem $S$ em dois hexágonos geodésicos retos isométricos.

Prova: Seja a uma curva simples em $S$ que conecta, por exemplo, as geodésicas da fronteira $\gamma_{2}$ e $\gamma_{3}$. Qualquer outra curva simples de $\gamma_{2}$ a $\gamma_{3}$ é homotópico a a com pontos extremos em $\gamma_{2}$ e $\gamma_{3}$. Pelo Teorema 4.11, a é homotópico a uma única geodésica 
perpendicular $a_{1}$ e esta perpendicular é simples. Daqui, temos três únicas geodésicas perpendiculares $a_{1}, a_{2}, a_{3}$ entre as geodésicas da fronteira.

Para ver que estas perpendiculares são disjuntas duas a duas, cortamos $S$ ao longo $a_{3}$, para obter uma superfície hiperbólica $A$ de assinatura $(0,2)$ com fronteira geodésica suave por partes. Novamente, pelo Teorema 4.11, existe uma geodésica simples perpendicular $a_{1}^{\prime}$ entre $\gamma_{2}$ e $\gamma_{3}$ em $A$. Pela unicidade de $a_{1}$ em $S$, temos que $a_{1}^{\prime}=a_{1}$. Portanto, $a_{1}$ não intersecta $a_{3}$ e o mesmo se cumpre para os outros pares de perpendiculares. Se cortamos $S$ ao longo de $a_{1}, a_{2}, a_{3}$ obtemos dois hexágonos geodésicos retos $G$ e $G^{\prime}$ em $\mathbb{H}$. Do Lema 1.49 obtemos que $G$ e $G^{\prime}$ são isométricos.

Corolário 4.14. Os pontos extremos das perpendiculares $a_{1}, a_{2}, a_{3}$ dividem cada geodésica da fronteira de $S$ em dois arcos do mesmo comprimento.

Teorema 4.15. Para quaisquer tripla de números positivos $l_{1}, l_{2}, l_{3}$ existe uma única calça $Y$ com geodésicas da fronteira $\gamma_{1}, \gamma_{2}, \gamma_{3}$ de comprimentos $l\left(\gamma_{i}\right)=l_{i}, i=1,2,3$.

Prova: Isto segue-se devido a existência e unicidade, a menos de isometrias, de hexágonos retos de comprimentos não adjacentes $l_{1} / 2, l_{2} / 2, l_{3} / 2$.

Proposição 4.16 (Semi-colares). Seja $Y$ uma calça com geodésicas da fronteira $\gamma_{1}, \gamma_{2}, \gamma_{3}$. Os conjuntos

$$
\mathcal{C}^{*}\left[\gamma_{i}\right]=\left\{p \in Y \mid \sinh \left(\rho\left(p, \gamma_{i}\right)\right) \sinh \frac{1}{2} l\left(\gamma_{i}\right) \leq 1\right\}, \quad i=1,2,3
$$

são disjuntos dois a dois e cada um é homeomorfo a $[0,1] \times \mathcal{S}^{1}$. Para qualquer $p \in \mathcal{C}^{*}\left[\gamma_{i}\right]$ existe em $\mathcal{C}^{*}\left[\gamma_{i}\right]$ uma única perpendicular a $\gamma_{i}, i=1,2,3$.

Prova: Decompomos $Y$ em dois hexágonos geodésicos retos $G$ e $G^{\prime}$ com lados rotulados e parametrizados como em (4.4). Então temos que $c_{i}=\frac{1}{2} l\left(\gamma_{i}\right)=c_{i}^{\prime}, i=1,2,3$. Em $G$ (e similarmente em $G^{\prime}$ definimos)

$$
\mathcal{C}_{i}=\left\{p \in G \mid \sinh \rho\left(p, c_{i}\right) \sinh c_{i} \leq 1\right\}, \quad i=1,2,3 .
$$

Para ver que os $\mathcal{C}_{i}$ são disjuntos dois a dois, desenhamos as perpendiculares comuns entre lados opostos, por exemplo $h$ de $c_{3}$ ao lado entre $c_{1}$ e $c_{2}$. Pelo Teorema $1.51(i)$ obtemos

$$
\sinh c_{k} \sinh \rho\left(c_{k}, h\right)>1, \quad k=1,2
$$

Daqui, $h$ separa $\mathcal{C}_{1}$ e $\mathcal{C}_{2}$ e similarmente $\mathcal{C}_{1}, \mathcal{C}_{3}$ e $\mathcal{C}_{2}, \mathcal{C}_{3}$ são separados. Além disso, desta separação cada $\mathcal{C}_{i}$ é varrido por arcos geodésicos disjuntos dois a dois de comprimento 
$d_{i}$ emanando perpendicularmente de $c_{i}$, onde $\sinh c_{i} \sinh d_{i}=1, i=1,2,3$. O mesmo cumpre para os correpondentes domínios em $G^{\prime}$.

Definição 4.17. Um homeomorfismo $\phi: A \rightarrow B$ entre dois espaços métricos $\left(A, d_{1}\right)$ e $\left(B, d_{2}\right)$ é uma q-quase-isometria $(q \geq 1)$ se $\frac{1}{q} d_{1}(x, y) \leq d_{2}(\phi x, \phi y) \leq d_{1}(x, y)$. Denotamos por $q[\phi]$ o infimo sobre todos os $q^{\prime}$ para os quais $\varphi$ é uma $q^{\prime}$-quase-isometria.

Sejam $G$ e $\tilde{G}$ dois hexágonos geodésicos retos em $\mathbb{H}$ cujos lados são parametrizados como em (4.4). Seja $p_{0}$ um vértice comum dos lados $\alpha_{2}$ e $c_{1}$ em $G$ e $p_{0}$ o vértice comum dos lados $\tilde{\alpha}_{2}$ e $\tilde{c}_{1}$ em $\tilde{G}$. As diagonais em $p_{0}$ e $\tilde{p}_{0}$ triangulam os hexágonos como na Figura 4.2 .
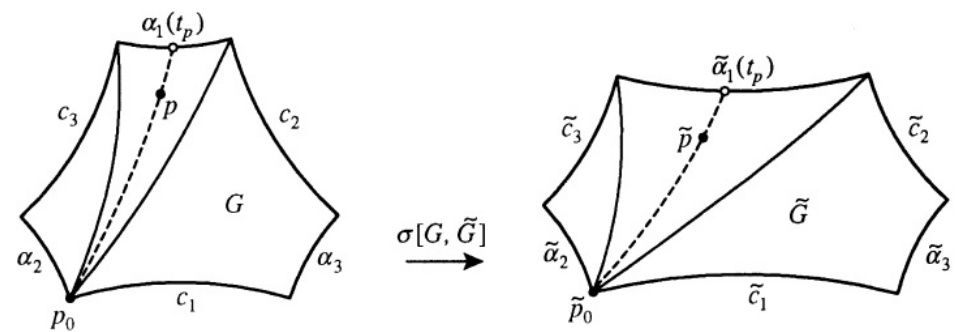

Figura 4.2:

Definimos uma aplicação $\sigma[G, \tilde{G}]: G \rightarrow \tilde{G}$ da seguinte maneira:

Para cada ponto $p \in G, p \neq p_{0}$, existe um único lado $\alpha_{i}$ (respectivamente, $c_{i}$ ) e um único parâmetro $t_{p} \in[0,1]$ tal que $p$ pertence ao segmento geodésico de $p_{0}$ a $p_{*}=\alpha_{i}\left(t_{p}\right)$ (de $p_{0}$ a $p_{*}=c_{i}\left(t_{p}\right)$ ). Seja $\sigma[G, \tilde{G}](p)$ o ponto $\tilde{p} \in \tilde{G}$ que esta no segmento geodésico de $\tilde{p}_{0}$ a $\tilde{p}_{*}=\tilde{\alpha}_{i}\left(t_{p}\right)\left(\right.$ de $\tilde{p}_{0}$ a $\left.\tilde{p}_{*}=\tilde{c}_{i}\left(t_{p}\right)\right)$ satisfazendo

$$
\frac{\rho\left(\tilde{p}_{0}, \tilde{p}\right)}{\rho\left(\tilde{p}_{0}, \tilde{p}_{*}\right)}=\frac{\rho\left(p_{0}, p\right)}{\rho\left(p_{0}, p_{*}\right)}
$$

e completamos a definição, pondo $\sigma[G, \tilde{G}]\left(p_{0}\right)=\tilde{p}_{0}$. Então, $\sigma[G, \tilde{G}]$ é uma quaseisometria que preserva a parametrização das fronteiras.

Definição 4.18. Sejam $Y$ e $\tilde{Y}$ arbitrárias $Y$-peças na forma básica (Proposição 4.13) e sejam $G$ e $G^{\prime}\left(\tilde{G}\right.$ e $\left.\tilde{G}^{\prime}\right)$ os hexágonos correspondentes. Definimos $\sigma[Y, \tilde{Y}]: Y \rightarrow \tilde{Y}$ por

$$
\sigma[Y, \tilde{Y}]= \begin{cases}\sigma[G, \tilde{G}] & \text { em } G \\ \sigma\left[G^{\prime}, \tilde{G}^{\prime}\right] & \text { em } G^{\prime}\end{cases}
$$

Observação 4.19. $\sigma[Y, \tilde{Y}]$ preserva a parametrização 4.6 das geodésicas da fronteira pois $\sigma[G, \tilde{G}]$ e $\sigma\left[G^{\prime}, \tilde{G}^{\prime}\right]$ preservam a parametrização das fronteiras. 
Definição 4.20. Uma superfície de Riemann compacta de assinatura $(0,4)$ é chamada uma $X$-peça.

$X$-peças são obtidas colando duas $Y$-peças ao longo de duas geodésicas da fronteira do mesmo comprimento. Um parâmetro adicional aparece: parâmetro de torção. Sejam $Y$ e $Y^{\prime}$ duas $Y$-peças dadas na forma básica com geodésicas da fronteira $\gamma_{i}, \gamma_{i}^{\prime}$ parametrizadas em $\mathcal{S}^{1}, i=1,2,3$, e suponhamos que $l\left(\gamma_{1}\right)=l\left(\gamma_{1}^{\prime}\right)$. Então, para qualquer número real $\alpha$ obtemos uma $X$-peça $X^{\alpha}$ pela identificação

$$
\gamma_{1}(t)=\gamma_{1}^{\prime}(\alpha-t)=: \gamma^{\alpha}(t) \quad t \in \mathcal{S}^{1}
$$

O número $\alpha$ é chamado parâmetro de torção, e escrevemos $X^{\alpha}:=Y+Y^{\prime} \bmod 4.8$. A fim de distinguir os elementos da família $\left\{X^{\alpha}\right\}, \alpha \in \mathbb{R}$ um de cada outro, utilizamos $X^{\alpha}$ para designar ao par ordenado $\left(\alpha, Y+Y^{\prime} \bmod (4.8)\right)$ : a $X$-peça marcada com $\alpha$.

Proposição 4.21. Todo X-peça pode ser construída como acima.

Prova: Seja $X$ uma $X$-peça dada, logo existe uma curva fechada simples homotópicamente não trivial e não homotópica a uma geodésica fronteira em $X$. Tomando tal curva e substituindo pela geodésica fechada simples em sua classe de homotopía livre, obtemos uma decomposição de $X$ em duas $Y$-peças.

Definição 4.22. Seja $X^{\alpha}$ com geodésica $\gamma^{\alpha}$ como em (4.8). O conjunto

$$
\mathcal{C}\left[\gamma^{\alpha}\right]=\left\{p \in X^{\alpha} \mid \rho\left(p, \gamma^{\alpha}\right) \leq w\right\}
$$

onde $w=\operatorname{arcsinh}\left\{1 / \sinh \frac{1}{2} l\left(\gamma^{\alpha}\right)\right\}$ é chamado colar ao redor de $\gamma^{\alpha}$ e wé sua largura.

Pela Proposição 4.16, para $p \in \mathcal{C}\left[\gamma^{\alpha}\right]$, existe uma única perpendicular em $\mathcal{C}\left[\gamma^{\alpha}\right]$ de $p$ a $\gamma^{\alpha}$ a qual intersecta $\gamma^{\alpha}$ no ponto $\gamma^{\alpha}\left(t_{p}\right)$ para um unicamente determinado $t_{p} \in \mathcal{S}^{1}$. Seja $\rho_{p}=\varepsilon \rho\left(p, \gamma^{\alpha}\right)$ a distância dirigida $\operatorname{com} \varepsilon=-1$ se $p \in Y$ e $\varepsilon=+1$ se $p \in Y^{\prime}$.

Definição 4.23. Para $p \in \mathcal{C}\left[\gamma^{\alpha}\right]$, como acima, $(\rho, t)=\left(\rho_{p}, t_{p}\right) \in[-w, w] \times \mathcal{S}^{1}$ é o par de coordenadas de Fermi de p com respeito a $\gamma^{\alpha}$.

Pela Proposição 4.16 estas coordenadas definem um homeomorfismo $\operatorname{de} \mathcal{C}\left[\gamma^{\alpha}\right]$ sobre o anel $[-w, w] \times \mathcal{S}^{1}$. Na família $\left\{X^{\alpha}\right\}, \alpha \in \mathbb{R}$ consideramos $X^{0}$ como uma superfície base e escrevemos $X^{0}=X, \gamma^{0}=\gamma$. O próximo passo é definir um homeomorfismo $\tau^{\alpha}: X \rightarrow X^{\alpha}$ (mostrado na Figura 4.3 )

De agora em diante, $\pi^{\alpha}: Y \cup Y^{\prime} \rightarrow X^{\alpha}$ denotará a projeção natural, e para $\alpha=0$, escrevemos $\pi^{0}=\pi$. Notar que a condição de colagem (4.8) em coordenadas de Fermi 


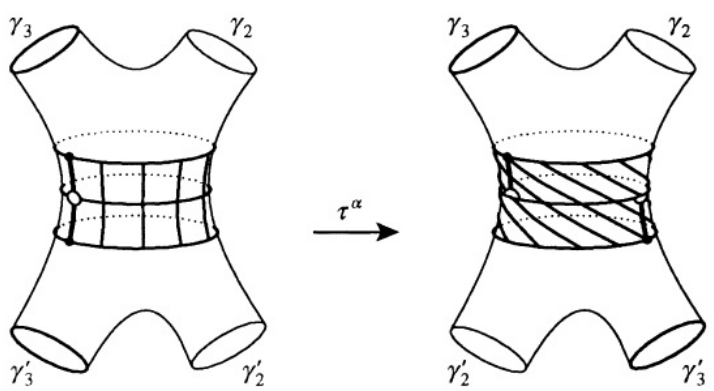

Figura 4.3: Homeomorfismo $\tau^{\alpha}$.

quer dizer o seguinte: se $p \in \mathcal{C}[\gamma]$ tem coordenadas $(\rho, t)$ e $\rho \leq 0$, então pela convenção de acima, $\pi^{-1}(p) \in Y$. Seguemos que $\pi^{\alpha} \circ \pi^{-1}(p) \in \mathcal{C}\left[\gamma^{\alpha}\right]$ tem exatamente as mesmas coordenadas $(\rho, t)$ com respeito a $\gamma^{\alpha}$ (pois $\gamma^{\alpha}(t)=\gamma_{1}(t)$ ). Se $\rho \geq 0$, então $\pi^{-1}(p) \in Y^{\prime}$, e seguemos que $\pi^{\alpha} \circ \pi^{-1}(p)$ tem coordenadas $(\rho, \alpha+t)$. Seguemos que a aplicação $T^{\alpha}: \mathcal{C}[\gamma] \rightarrow \mathcal{C}\left[\gamma^{\alpha}\right]$ definida por

$$
T^{\alpha}(\rho, t)=\left(\rho, t+\alpha \frac{w+\rho}{2 w}\right)
$$

( $w$ é a largura comum dos colares $\mathcal{C}[\gamma]$ e $\mathcal{C}\left[\gamma^{\alpha}\right]$ ) coincide com $\pi^{\alpha} \circ \pi^{-1}$ na fronteira de $\mathcal{C}[\gamma]$. Definindo

$$
\tau^{\alpha}= \begin{cases}T^{\alpha} & \text { em } \mathcal{C}[\gamma] \\ \pi^{\alpha} \circ \pi^{-1} & \text { em } X-\mathcal{C}[\gamma]\end{cases}
$$

obtemos um homeomorfismo $\tau^{\alpha}: X \rightarrow X^{\alpha}$. As aplicações $T^{\alpha}$ e $\tau^{\alpha}$ são chamadas homeomorfismos de torção.

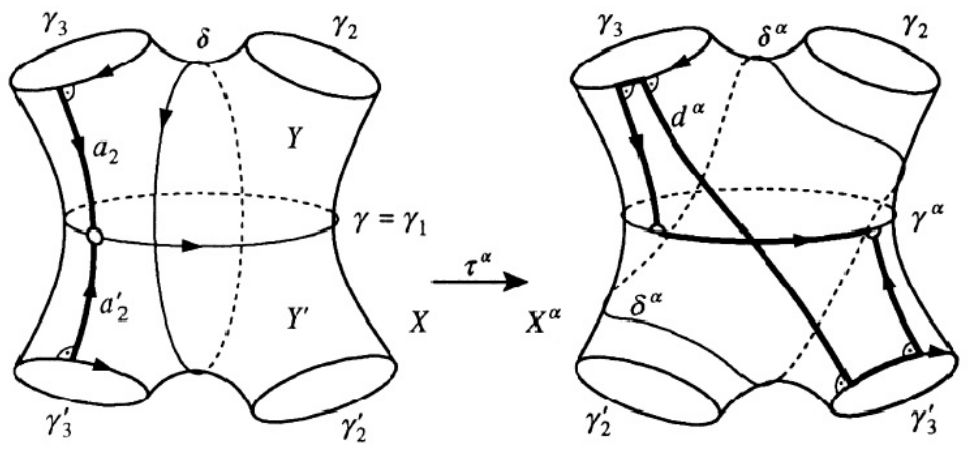

Figura 4.4:

O próximo passo é introduzir aplicações como acima, mas agora de $X^{\alpha}$ sobre se mesmo. Empezamos em $\mathcal{C}\left[\gamma^{\alpha}\right]$ com a aplicação $\mathscr{T}: \mathcal{C}\left[\gamma^{\alpha}\right] \rightarrow \mathcal{C}\left[\gamma^{\alpha}\right]$ definida por

$$
\mathscr{T}(\rho, t)=\left(\rho, t+\frac{w+\rho}{2 w}\right)
$$


Definição 4.24. A aplicação $\mathscr{D}: X^{\alpha} \rightarrow X^{\alpha}$ definida por

$$
\mathscr{D}= \begin{cases}\mathscr{T} & \text { em } \mathcal{C}\left[\gamma^{\alpha}\right] \\ \text { Id } & \text { em } X^{\alpha}-\mathcal{C}\left[\gamma^{\alpha}\right]\end{cases}
$$

é chamada uma torção elementar de Dehn. Uma torção de Dehn de $X^{\alpha}$ de ordem $m, m \in \mathbb{Z}$, é um homeomorfismo de $X^{\alpha}$ isotópico a $\mathscr{D}^{m}$ o qual fixa a fronteira $\partial X$ pontoalmente.

Em $X$, seja $d=a_{2}^{\prime} a_{2}^{-1}$ (a curva parametrizada $a_{2}^{\prime}$ seguida da parametrização inversa de $a_{2}$ ). Então $d$ é uma curva perpendicular de $\gamma_{3}^{\prime}$ a $\gamma_{3}$. Na classe de homotopia livre de $d \gamma_{3} d^{-1} \gamma_{3}^{\prime}$ existem curvas fechadas simples tal que $d \gamma_{3} d^{-1} \gamma_{3}^{\prime}$ é homotópico a uma geodésica fechada simples $\delta$ a qual separa $\gamma_{2}$ e $\gamma_{2}^{\prime}$ de $\gamma_{3}$ e $\gamma_{3}^{\prime}$ (Ver Figura 4.4). Como uma segunda curva deste tipo, seja $\eta$ a geodésica na classe de homotopia de $\mathscr{D}(\delta)$ onde $\mathscr{D}: X \rightarrow X$ é a torção de Dehn. Resumindo, lembraremos que

$$
\begin{array}{cc}
\delta \text { é homotópico a } d \gamma_{3} d^{-1} \gamma_{3}^{\prime} & \text { onde } d=a_{2}^{\prime} a_{2}^{-1} \\
\eta \text { é homotópico a } \bar{d} \gamma_{3} \bar{d}^{-1} \gamma_{3}^{\prime} & \text { onde } \bar{d}=a_{2}^{\prime} \gamma_{1} a_{2}^{-1} .
\end{array}
$$

Definição 4.25. Em $X^{\alpha}$, seja $\delta^{\alpha}$ e $\eta^{\alpha}$ as geodésicas fechadas e simples na classe de homotopia livre de $\tau^{\alpha}(\delta)$ e $\tau^{\alpha}(\eta)$ onde $\tau^{\alpha}: X \rightarrow X^{\alpha}$ é o homeomorfismo de torção.

Proposição 4.26. Para a família $\left\{X^{\alpha}\right\}, \alpha \in \mathbb{R}$ seja $F$ a função

$$
F(\alpha)=\sinh \frac{1}{2} \gamma_{3} \sinh \frac{1}{2} \gamma_{3}^{\prime}\left\{\sinh a_{2} \sinh a_{2}^{\prime} \cosh (\alpha \gamma)+\cosh a_{2} \cosh a_{2}^{\prime}\right\}-\cosh \frac{1}{2} \gamma_{3} \cosh \frac{1}{2} \gamma_{3}^{\prime}
$$

onde utilizamos a convenção $\gamma_{3}=l\left(\gamma_{3}\right), \gamma_{3}^{\prime}=l\left(\gamma_{3}^{\prime}\right)$. Então $\cosh \frac{1}{2} \delta^{\alpha}=F(\alpha) e \cosh \frac{1}{2} \eta^{\alpha}=$ $F(\alpha+1)$.

Prova: Pela definição de $\tau^{\alpha}$, a curva $\tau^{\alpha}(d)$ é homotópica (com pontos extremos estando em $\gamma_{3}^{\prime}$ e $\gamma_{3}$ ) a uma curva $a_{2}^{\prime} b a_{2}^{-1}$ em $X^{\alpha}$, onde $b$ é um arco parametrizado em $\gamma^{\alpha}$ de comprimento $|\alpha| l\left(\gamma^{\alpha}\right)=|\alpha l(\gamma)|=|\alpha \gamma|$. Utilizando o Teorema 4.6, na classe de homotopia de $\tau^{\alpha}(d)$ existe uma perpendicular $d^{\alpha}$ (Ver Figura 4.4). Levantando estas curvas ao recobrimento universal de $X^{\alpha}$ (sem mudar a notação) obtemos um hexágono reto com autointerseção como na Figura 4.5. Do Teorema 1.53 obtemos

$$
\cosh d^{\alpha}=\sinh a_{2} \sinh a_{2}^{\prime} \cosh (\alpha \gamma)+\cosh a_{2} \cosh a_{2}^{\prime}
$$

Isto relaciona $|\alpha|$ com o comprimento de $d^{\alpha}$. Para relacioná-lo ao comprimento da geodésica fechada $\delta^{\alpha}$, notar que $\delta^{\alpha}$ é livremente homotópica à curva fechada $d^{\alpha} \gamma_{3}\left(d^{\alpha}\right)^{-1} \gamma_{3}^{\prime}$ 
e $\delta^{\alpha}$ não intersecta $d^{\alpha}$. Daqui, $\delta^{\alpha}$ é uma curva perpendicular simples de $\gamma_{3}^{\prime}$ a $\gamma_{3}$ na calça $Y$ em $X^{\alpha}$ cujas geodésicas da fronteira são $\gamma_{3}^{\prime}, \gamma_{3}$ e $\delta^{\alpha}$. Portanto, podemos decompor $Y$ em dois hexágonos retos e aplicar a Proposição 1.52 para obter

$$
\cosh \frac{1}{2} \delta^{\alpha}=\sinh \frac{1}{2} \gamma_{3} \sinh \frac{1}{2} \gamma_{3}^{\prime} \cosh d^{\alpha}-\cosh \frac{1}{2} \gamma_{3} \cosh \frac{1}{2} \gamma_{3}^{\prime} .
$$

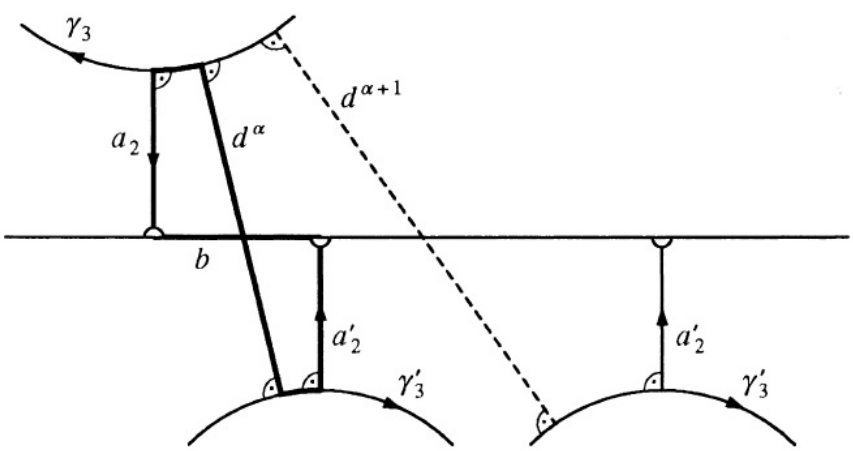

Figura 4.5:

Assim, de 4.10) e 4.11) obtemos

$$
\cosh \frac{1}{2} \delta^{\alpha}=F(\alpha)
$$

Para provar a fórmula para $\eta$, observamos que $\eta^{\alpha}$ em $X^{\alpha}$ tem o mesmo comprimento que $\delta^{\alpha+1}$ em $X^{\alpha+1}$.

Podemos expressar $a_{2}$ e $a_{2}^{\prime}$ em termos dos comprimentos de $\gamma$ e das geodésicas da fronteira, assim da Proposição 4.26 temos o resultado seguinte

Proposição 4.27. Na família $\left\{X^{\alpha}\right\}, \alpha \in \mathbb{R}$, temos

$$
\begin{aligned}
\cosh \frac{1}{2} \delta^{\alpha} & =u+\nu \cosh (\alpha \gamma) \\
\cosh \frac{1}{2} \eta^{\alpha} & =u+\nu \cosh ((\alpha+1) \gamma)
\end{aligned}
$$

onde os coeficientes u e $\nu$ são funcões analíticas reais dos comprimentos de $\gamma, \gamma_{2}, \gamma_{3}, \gamma_{2}^{\prime}$ e $\gamma_{3}^{\prime}$ independentes de $\alpha$. Além disso $\nu>0$.

Até o momento temos fixado os comprimentos de $\gamma$ e das geodésicas da fronteira. Se deixarmos eles variam também, então cada $X^{\alpha}$ determina um vector

$$
L=L\left[\alpha, \gamma, \gamma_{2}, \gamma_{3}, \gamma_{2}^{\prime}, \gamma_{3}^{\prime}\right]:=\left(\gamma^{\alpha}, \delta^{\alpha}, \eta^{\alpha}, \gamma_{2}, \gamma_{3}, \gamma_{2}^{\prime}, \gamma_{3}^{\prime}\right) \in \mathbb{R}^{7}
$$

para todo $\alpha$. Os parâmetros $\alpha, \ldots, \gamma_{3}^{\prime}$ preenchem $\mathbb{R} \times \mathbb{R}_{+}^{5}$ e os correspondentes valores de $L$ preenchem uma subvariedade analítica real $\mathcal{L}$ de dimensão 6 em $\mathbb{R}^{7}$. 
Proposição 4.28. Existe uma vizinhança aberta $U_{\mathcal{L}}$ de $\mathcal{L}$ em $\mathbb{R}^{7}$ e uma função analítica real $\mathscr{A}: U_{\mathcal{L}} \rightarrow \mathbb{R}$ tal que $\alpha=\mathscr{A}\left(L\left[\alpha, x_{1}, \ldots, x_{5}\right]\right)$ para todo $\left(\alpha, x_{1}, \ldots, x_{5}\right) \in \mathbb{R} \times \mathbb{R}_{+}^{5}$

Prova: Como a função cosh é uma função par então a função $z \mapsto \cosh \sqrt{z}, z \in \mathbb{C}$ é uma função holomorfa em $\mathbb{C}$. Sua restrição a $\mathbb{R}$ tem derivadas positivas no intervalo ]$-\pi^{2}, \infty[$. Portanto, sua inversa

$$
g(t)= \begin{cases}-(\arccos t)^{2} & \text { para }-1 \leq t \leq 1 \\ (\operatorname{arccosh} t)^{2} & \text { para } t \geq 1\end{cases}
$$

é analítica real no intervalo ] - 1, $\infty$ [. Com Proposição 4.27 temos a solução explicita

$$
\alpha=\frac{1}{2 l^{2}(\gamma)}\left[g\left(\frac{1}{\nu}\left(\cosh \left(\frac{1}{2} \eta^{\alpha}\right)-u\right)\right)-g\left(\frac{1}{\nu}\left(\cosh \left(\frac{1}{2} \delta^{\alpha}\right)-u\right)\right)\right]-\frac{1}{2}
$$

onde $u$ e $\nu$ são analíticas reais e $\nu$ é positivo. Se $L \in \mathcal{L}$, então ambos argumentos de $g$ na fórmula acima são positivos. Como $g$ é analítica real em um intervalo aberto que contém $[0, \infty[$, a expresão de 4.13 ) é bem definida e analítica real em uma vizinhança de $\mathcal{L}$.

Da Proposição 4.28, temos em particular que

$$
\alpha=\mathscr{A}\left(\gamma^{\alpha}, \delta^{\alpha}, \eta^{\alpha}, \gamma_{2}, \gamma_{3}, \gamma_{2}^{\prime}, \gamma_{3}^{\prime}\right)
$$

O próximo resultado nos permite decompor toda superfície de Riemann fechada de gênero $g \geq 2$ em calças.

Proposição 4.29. Seja S uma superfície de Riemann fechada de gênero $g \geq 2$, e sejam $\gamma_{1}, \ldots, \gamma_{n}$ geodésicas fechadas, simples e disjuntas em S. Então tem-se:

(i) $m \leq 3 g-3$.

(ii) Existem geodésicas fechadas e simples $\gamma_{m+1}, \ldots, \gamma_{3 g-3}$ as quais, junto com $\gamma_{1}, \ldots, \gamma_{m}$ decompõem $S$ em calças.

(iii) Os colares $\mathcal{C}\left(\gamma_{i}\right)=\left\{p \in S \mid \rho\left(p, \gamma_{i}\right) \leq w\left(\gamma_{i}\right)\right\}$ de largura $w\left(\gamma_{i}\right)=\operatorname{arcsinh}\left\{1 / \sinh \left(\frac{1}{2} l\left(\gamma_{i}\right)\right)\right\}$ são disjuntos dois a dois para $i=1, \ldots, 3 g-3$.

Prova: Cortamos $S$ ao longo de $\gamma_{1}, \ldots, \gamma_{m}$. Cada componente $S^{\prime}$ da superfície obtida é uma superfície hiperbólica de assinatura $\left(g^{\prime}, n^{\prime}\right)$. Pela hipótesis temos que $g^{\prime} \geq 1$ ou $g^{\prime}=0, n^{\prime} \geq 3$.

Suponhamos que $S^{\prime}$ não tem assinatura $(0,3)$. Então $S$ contém uma curva fechada simples homotopicamente não trivial, $\gamma_{m+1}$, a qual não é homotopica a uma componente da fronteira de $S^{\prime}$. Pela Proposição 2.37 podemos assumir que $\gamma_{m+1}$ é uma geodéscia 
fechada. Cortamos $S^{\prime}$ ao longo de $\gamma_{m+1}$ e continuamos. Depois de um número finito de passos, $S$ é decomposto em calças. A característica de Euler nos diz que o número de calças é $2 g-2$. Isto da $(i)$ e $(i i)$.

Como $S$ é decomposto em calças, a afirmação (iii) segue-se da Proposição 4.16

\subsection{2 $\mathrm{O}$ caso da assinatura $(1,1)$}

Na seção anterior obtimos uma $X$-peça colando duas $Y$-peças distintas ao longo de geodésicas com o mesmo comprimento. Agora veremos o caso quando colamos as geodésicas do mesmo comprimento mas de uma mesma calça.

Seja $Y$ uma calça, dada na forma básica, com geodésicas da fronteira $\gamma_{1}, \gamma_{2}, \gamma_{3}$ e suponhamos que $\gamma_{1}$ e $\gamma_{2}$ têm o mesmo comprimento. Então a condição de colagem

$$
\gamma_{1}(t)=\gamma_{2}(\alpha-t)=: \gamma_{\alpha}(t), \quad t \in \mathcal{S}^{1}
$$

nos dá uma superfície de Riemann compacta de assinatura $(1,1) ; Q_{\alpha}:=Y \bmod$ (4.15). Novamente distinguimos os elementos da família $\left\{Q_{\alpha}\right\}, \alpha \in \mathbb{R}$ um de cada outro, os consideramos como pares ordenados $(\alpha, Y \bmod (4.15)$ ) e omitimos $\alpha$ quando $\alpha=0$. Consideremos uma cópia $Y^{\prime}$ de $Y$. Mudamos o nome das geodésicas de tal maneira que temos uma isometria satisfazendo

$$
m\left(\gamma_{1}^{\prime}(t)\right)=\gamma_{2}(t), \quad m\left(\gamma_{2}^{\prime}(t)\right)=\gamma_{3}(t), \quad m\left(\gamma_{3}^{\prime}(t)\right)=\gamma_{1}(t)
$$

$t \in \mathcal{S}^{1}$. A condição de colagem

$$
\gamma_{1}(t)=\gamma_{1}^{\prime}(\alpha-t)=: \tilde{\gamma}^{\alpha}(t), \quad t \in \mathcal{S}^{1}
$$

define a $X$-peça $X^{\alpha}:=Y+Y^{\prime} \bmod$ (4.16). Denotamos por $\pi_{\alpha}: Y \rightarrow Q_{\alpha}$ a projeção natural e definimos $J_{\alpha}: X^{\alpha} \rightarrow Q_{\alpha}$ por

$$
J_{\alpha}= \begin{cases}\pi_{\alpha} & \text { em } Y \\ \pi_{\alpha} \circ m & \text { em } Y^{\prime}\end{cases}
$$

Esta aplicação é uma imersão isométrica. Pela Proposição 4.16, os semi-colares $\mathcal{C}^{*}\left[\gamma_{1}\right]$ $\left(\mathcal{C}^{*}\left[\gamma_{2}\right]\right)$ em $Y$ formado pelos pontos a distância $\leq w=\operatorname{arcsinh}\left\{1 / \sinh \frac{1}{2} \gamma_{1}\right\}$ de $\gamma_{1}$ (respectivamente, $\gamma_{2}$ ) são disjuntos. Segue-se que os colares

$$
\mathcal{C}\left[\tilde{\gamma}^{\alpha}\right]=\left\{p \in X^{\alpha} \mid \rho\left(p, \tilde{\gamma}^{\alpha}\right) \leq w\right\}, \quad \mathcal{C}\left[\gamma_{\alpha}\right]=\left\{p \in Q_{\alpha} \mid \rho\left(p, \gamma_{\alpha}\right) \leq w\right\}
$$


são isométricos, onde $J_{\alpha}: \mathcal{C}\left[\tilde{\gamma}^{\alpha}\right] \rightarrow \mathcal{C}\left[\gamma_{\alpha}\right]$ é a isometria. Em todos os colares, denotamos por $(\rho, t)$ as coordenadas de Fermi.

Similarmente, como no caso das $X$-calças, seja $T_{\alpha}: \mathcal{C}[\gamma] \rightarrow \mathcal{C}\left[\gamma_{\alpha}\right]$ dado por

$$
T_{\alpha}(\rho, t)=\left(\rho, t+\alpha \frac{w+\rho}{2 w}\right)
$$

e definimos o homeomorfismo de torção $\tau_{\alpha}: Q \rightarrow Q_{\alpha}$ como segue

$$
\tau_{\alpha}= \begin{cases}T_{\alpha} & \text { em } \mathcal{C}[\gamma] \\ \pi_{\alpha} \circ \pi^{-1} & \text { em } Q-\mathcal{C}[\gamma]\end{cases}
$$

Seja $\tau^{\alpha}: X \rightarrow X^{\alpha}$ o homeomorfismo correspondente de (4.9). Nestas condições temos o seguinte resultado.

Proposição 4.30. Seja $\mu$ uma curva fechada em $X$. Então as curvas $\tau_{\alpha} \circ J(\mu)$ e $J_{\alpha} \circ \tau^{\alpha}(\mu)$ em $Q_{\alpha}$ são homotópicas.

Agora, vamos calcular o parâmetro de torção $\alpha$ em termos dos comprimentos das geodésicas fechadas:

Denotamos por $\tilde{\delta}^{\alpha}$ e $\tilde{\eta}^{\alpha}$ em $X^{\alpha}$ as geodésicas da Definição 4.25 as quais determinam o parâmetro de torção de $X^{\alpha}$ e definamos as seguintes curvas em $Q_{\alpha}$

$$
\delta_{\alpha}=J_{\alpha}\left(\tilde{\delta}^{\alpha}\right), \quad \eta_{\alpha}=J_{\alpha}\left(\tilde{\eta}^{\alpha}\right)
$$

Pela Proposição $4.30, \delta_{\alpha}$ e $\eta_{\alpha}$ são geodésicas fechadas na classe de homotopia livre das curvas $\tau_{\alpha}(\delta)$ e $\tau_{\alpha}(\eta)$. Como $Q_{\alpha}$ e $X^{\alpha}$ têm o mesmo parâmetro de torção, e desde que $J_{\alpha}$ preserva comprimentos obtemos o análogo da equação (4.14), $\alpha=\mathscr{A}\left(\gamma_{\alpha}, \delta_{\alpha}, \eta_{\alpha}, \gamma_{2}, \gamma_{3}, \gamma_{3}, \gamma_{2}\right)$, onde $\mathscr{A}$ é a função como na Proposição 4.28 .

\subsubsection{Grafos}

Nesta subseção definiremos um grafo cúbico marcado e o utilizaremos como esqueleto combinatório para o colagem de $Y$-peças para construir superfícies de Riemann de gênero $g \geq 2$.

Um grafo consiste de um conjunto de vértices (representados como pontos) e arestas (representados como segmentos de linhas). Denotamos por $\# G$ a cardinalidade do conjunto de vértices. Cada aresta conecta dois vértices, uma aresta pode conectar um vértice com ele mesmo. Vamos olhar cada aresta como a união de duas semi-arestas, com cada semi-aresta saindo dos dois vértices conectados. $G$ é chamado 3-regular se 
todo vértice tem três semi-arestas saindo dele.

Na construção de superfícies de Riemann compactas cada $Y$-peça com suas três geodésicas da fronteira será interpretada como um vértice com seus três semi-arestas. $G$ é conexo, se para cada par de vértices distintos $x$ e $y$ encontramos uma sequência $x_{1}, \ldots, x_{n}$ de vértices com $x_{1}=x$ e $x_{n}=y$ tal que para cada par $x_{i}, x_{i+1}$ é conectado por uma aresta, $i=1, \ldots, n-1$.

Definição 4.31. Um grafo cúbico é um grafo conexo 3-regular.

Seja $G$ um grado cúbico fixado, então $\# G$ é um número par que escrevemos na forma $\# G=2 g-2,(g \geq 2)$. Como $G$ é 3-regular, ele deve ter $3 g-3$ arestas. Utilizamos a seguinte notação:

Os vértices e arestas de $G$ são denotados por

$$
y_{1}, \ldots, y_{2 g-2} \quad \text { e } \quad c_{1}, \ldots, c_{3 g-3}
$$

Para cada $y_{i}$, as três semi-arestas são denotadas por $c_{i \mu}, \mu=1,2,3$. Se $c_{i \mu}$ e $c_{j \nu}$ são duas semi-arestas da aresta $c_{k}$ escrevemos $c_{k}=\left(c_{i \mu}, c_{j \nu}\right)$. Desta forma $G$ é totalmente descrito pela lista

$$
c_{k}=\left(c_{i \mu}, c_{j \nu}\right), \quad k=1, \ldots, 3 g-3 .
$$

Para a construção de superfícies de Riemann é prático ver a lista (4.19) como um grafo. De fato, assumamos que símbolos $c_{i \mu}$ são dados, com $i=1, \ldots, 2 g-2$ e $\mu=1,2,3$. Então escrevemos uma lista 4.19 de pares ordenados na qual cada símbolo acontece uma só vez, e seja $y_{i}=\left\{c_{i 1}, c_{i 2}, c_{i 3}\right\}$ para $i=1, \ldots, 2 g-2$ (os trios não são ordenados). Isto define um grafo 3-regular. Chamamos a este grafo admissível se o grafo for conexo.

Definição 4.32. Uma lista admissível como em (4.19) é chamado um grafo cúbico marcado.

Agora construímos superfícies de Riemann utilizando $Y$-peças como blocos de construção e grafos cúbicos marcados como esqueletos combinatórios. Fixamos um grafo cúbico marcado $G$ com vértices $y_{1}, \ldots, y_{2 g-2}$ e arestas $c_{1}, \ldots, c_{3 g-3}$ definidas pela relação (4.19). Logo, escolhemos

$$
L=\left(l_{1}, \ldots, l_{3 g-3}\right) \in \mathbb{R}_{+}^{3 g-3}, \quad A=\left(\alpha_{1}, \ldots, \alpha_{3 g-3}\right) \in \mathbb{R}^{3 g-3}
$$

e definimos a superfície de Riemann compacta $F(G, L, A)$ como segue:

Para cada vértice $y_{i}$ com semi-arestas $c_{i 1}, c_{i 2}, c_{i 3}$ saindo dele, associamos uma calça $Y_{i}$ 
com geodésicas da fronteira $\gamma_{i 1}, \gamma_{i 2}, \gamma_{i 3}$ parametrizados em $\mathcal{S}^{1}$ na forma básica, tal que para os pares da lista (4.19) temos

$$
l_{k}=l\left(\gamma_{i \mu}\right)=l\left(\gamma_{j \nu}\right), k=1, \ldots, 3 g-3 .
$$

Para cada par na lista então colamos $Y_{i}$ e $Y_{j}$ ao longo destas geodésicas pela seguinte relação

$$
\gamma_{i \mu}(t)=\gamma_{j \nu}\left(\alpha_{k}-t\right):=\gamma_{k}(t), \quad t \in \mathcal{S}^{1}=\mathbb{R} /[t \mapsto t+1]
$$

e pomos

$$
F=F(G, L, A)=Y_{1}+\ldots+Y_{2 g-2} \bmod 4.20 .
$$

Como $G$ é um grafo conexo, então $F$ é conexo. $F$ satisfaz as condições do Teorema 4.6 , assim $F$ é uma superfície de Riemann compacta. Calculando a característica de Euler, vemos que $F$ tem gênero $g$.

Definição 4.33. $(L, A)$ é a sequência de coordenadas de Fenchel-Nielsen da superfície de Riemann $F(G, L, A)$.

As geodésicas $\gamma_{1}, \ldots, \gamma_{3 g-3}$ em $F$ correspondentes às arestas $c_{1}, \ldots, c_{3 g-3}$ do grafo serão chamados parâmetros geodésicos de $F$.

Lema 4.34. Ver [3, pág.83].

Seja $\phi: S \rightarrow R$ um homeomorfismo de superfícies de Riemann compactas, e sejam $\gamma_{1}, \ldots, \gamma_{N}$ geodésicas fechadas, simples e disjuntas duas a duas em $S$. Então existe um homeomorfismo $\phi^{\prime}$ isotópico a $\phi$ tal que as curvas $\phi^{\prime}\left(\gamma_{1}\right) \ldots \phi^{\prime}\left(\gamma_{N}\right)$ são geodésicas fechadas em $R$.

Teorema 4.35. Seja G um grafo cúbico marcado fixo com $2 g-2$ vértices. Então $F(G, L, A)$ percorre todas as superfícies de Riemann de gênero $g$.

Prova: Seja $F_{0}=F\left(G, L_{0}, A_{0}\right)$ alguma superfície base fixada. Para toda superfície de Riemann compacta $S$ de gênero $g$ existe um homeomorfismo $\phi: F_{0} \rightarrow S$. Pelo Lema 4.34, $\phi$ pode ser escolhido tal que as imagens $\phi\left(\gamma_{i}\right)$ dos parâmetros geodésicos $\gamma_{1}, \ldots, \gamma_{3 g-3}$ de $F_{0}$ formam um sistema de geodésicas simples fechadas disjuntas dois a dois em $S$. Então $\phi$ mapea as calças de $F_{0}$ sobre as calças em $S$. Estas calças definem $S$ como uma superfície $S=F(G, L, A)$ para adequados $L$ e $A$. Desde que $\phi$ é um homeomorfismo, o grafo $G$ resulta o mesmo. 


\subsection{Coordenadas de Fenchel-Nielsen}

Para cada assinatura $(g, n) \operatorname{com} 2 g+n \geq 3$ fixamos uma superfície suave, compacta e orientável $F=F_{g, n}$ de gênero $g \operatorname{com} n$ furos tal que todas as componentes da fronteira são curvas fechadas suaves. $F$ é a superfície base para os homeomorfismos. Depois, introduziremos em $F$ uma estrutura de superfície de Riemann conveniente.

Definição 4.36. Uma superfície de Riemann marcada de assinatura $(g, n)$ é um par $(S, \varphi)$ onde $S$ é uma superfície de Riemann compacta de assinatura $(g, n)$ e $\varphi: F_{g, n} \rightarrow S$ é um homeomorfismo chamado homemorfismo marcado

Para simplificar a notação, escreveremos $S$ em vez de $(S, \varphi)$. Utilizando o Teorema 1.20 e a Proposição 4.7, podemos dar a seguinte definição de espaço de Teichmüller, equivalente à dada no Capítulo 3 , no caso de assinatura $(g, 0)$.

Definição 4.37. Duas superfícies marcadas $(S, \varphi)$ e $\left(S^{\prime}, \varphi^{\prime}\right)$ são equivalentes se existe uma isometria $m: S \rightarrow S^{\prime}$ tal que $\varphi^{\prime}$ e $m \circ \varphi$ são isotópicos. O conjunto de todas as classes de marcações equivalentes de assinatura $(g, n)$ é o espaço de Teichmüller de assinatura $(g, n)$ e é denotado por $T_{g, n}$. Se $n=0$ escrevemos, como no Capítulo $3, T_{g}$ em vez de $T_{g, 0}$.

Definição 4.38. Seja $(S, \varphi) \operatorname{com} \varphi: F_{g, n} \rightarrow S$ uma superfície de Riemann marcada. Para toda curva fechada homotopicamente não trivial $\beta$ em $F_{g, n}$ denotamos por $\beta(S)$ a única geodésica fechada em $S$ homotópica a $\varphi \circ \beta$.

Se $\mathcal{B}=\left\{\beta_{1}, \beta_{2}, \ldots\right\}$ é uma sequência finita ou infinita de tais curvas em $F_{g, n}$ denotamos por

$$
\mathcal{B}=\left\{l \beta_{1}(S), l \beta_{2}(S), \ldots\right\}
$$

a correspondente sequência de geodésicas fechadas em $S$ e definimos

$$
l \mathcal{B}(S)=\left\{l \beta_{1}(S), l \beta_{2}(S), \ldots\right\}
$$

Agora, nos centraremos no caso de superfícies sem fronteira e seja $g \geq 2$ fixado. Nosso objetivo nesta seção é provar que as superfícies $F(G, L, A)$ formam um modelo de $T_{g}$. Para isto definimos, para cada grafo cúbico marcado $G$, um sistema de curvas $\Omega_{G}$ na superfície base $F=F_{g, 0}$ o qual servirá para caracterizar as classes de marcações equivalentes.

Seja $G$ um grafo cúbico marcado dado pela lista $c_{k}=\left(c_{i \mu}, c_{j \nu}\right), k=1, \ldots, 3 g-3$. Vamos introduzir a seguinte estrutura hiperbólica na superfície base $F$. Para cada 
$i=1, \ldots, 2 g-2$ seja $Y_{i}$ uma $Y$-peça com geodésicas da fronteira $\gamma_{i \mu}, \mu=1,2,3$ de comprimento 1 , as $Y$-peças assim obtidas são coladas de tal maneira que se $c_{k}=\left(c_{i \mu}, c_{j \nu}\right)$ então

$$
\gamma_{i \mu}(t)=\gamma_{j \nu}(-t)=: \gamma_{k}(t), t \in \mathcal{S}^{1}
$$

Tomamos a superfície resultante como a superfície base $F$. Para simplificar a notação, denotamos também por $Y_{i}$ a imagem de $Y_{i}$ sob a projeção $Y_{1} \cup \ldots \cup Y_{2 g-2} \rightarrow F$.

Fixamos $k$. As duas $Y$-peças $Y_{i}$ e $Y_{j}$ as quais são coladas ao longo de $\gamma_{k}$ forma uma superfície hiperbólica em $F$ de diferentes assinaturas (as possíveis são $(1,1),(1,2),(0,4)$ e $(2,0))$. Para evitar a distinção entre os diferentes casos, definimos uma superfície $X^{k}$ junto com uma imersão isométrica $\iota_{k}: X^{k} \rightarrow F$ :

Sejam $Y^{i}$ e $Y^{j}$ distintas $Y$-peças, onde $Y^{i}$ é uma cópia de $Y_{i}$ e $Y^{j}$ é uma cópia de $Y_{j}$, as geodésicas da fronteira de $Y^{i}$ e $Y^{j}$ são denotadas por $\gamma_{r}^{i}$ e $\gamma_{s}^{j}, r, s=1,2,3$. A superfície $X^{k}$ é definida por

$$
X^{k}=Y^{i} \cup Y^{j} \bmod \left(\gamma_{\mu}^{i}(t)=\gamma_{\nu}^{j}(-t)=: \gamma^{k}(t), t \in \mathcal{S}^{1}\right)
$$

As isometrias naturais $Y^{i} \rightarrow Y_{i}, Y^{j} \rightarrow Y_{j}$ se projectam naturalmente a uma isometria $\iota_{k}: X^{k} \rightarrow F$.

Definição 4.39. Para $k=1, \ldots, 3 g-3$ pomos $\delta^{k}$ e $\eta^{k}$ em $X^{k}$ as geodésicas como na Definição 4.25 (neste caso $\alpha=0$ ). Denotamos as suas imagens em $F$ por $\delta_{k}=\iota_{k}\left(\delta^{k}\right)$ e $\eta_{k}=\iota_{k}\left(\eta^{k}\right)$. A sequência

$$
\Omega_{G}=\left\{\gamma_{1}, \ldots, \gamma_{3 g-3}, \delta_{1}, \ldots, \delta_{3 g-3}, \eta_{1}, \ldots, \eta_{3 g-3}\right\}
$$

é chamada o sistema canônico de curvas com respeito a $G$.

O seguinte teorema mostra que $\Omega_{G}$ pode ser utilizado para caracterizar a classe de isotopia de um homeomorfismo.

Teorema 4.40. Ver [3, pág.141].

Sejam $\varphi, \varphi^{\prime}: F \rightarrow$ S homeomorfismo marcados tal que $\varphi \circ \gamma_{k}$ é homotópico a $\varphi^{\prime} \circ \gamma_{k}$ e tal que $\varphi \circ \delta_{k}$ é homotópico a $\varphi^{\prime} \circ \delta_{k}, k=1, \ldots, 3 g-3$. Então $\varphi$ e $\varphi^{\prime}$ são isotópicos.

Como consequência deste teorema temos o seguinte corolário.

Corolário 4.41. Dois homeomorfismos $\varphi, \varphi^{\prime}: F \rightarrow S$ são homotópicos se e somente se são isotópicos.

De novo, seja $G$ um grafo com as relações $c_{k}=\left(c_{i \mu}, c_{j \nu}\right), k=1, \ldots, 3 g-3$, e consid- 
eremos as várias superfícies de Riemann $F^{L A}=F(G, L, A)$, onde

$$
(L, A)=\left(l_{1}, \ldots, l_{3 g-3}, \alpha_{1}, \ldots, \alpha_{3 g-3}\right) \in \mathbb{R}_{+}^{3 g-3} \times \mathbb{R}^{3 g-3}=: \mathcal{R}^{6 g-6}
$$

Voltamo-nos este conjunto em um modelo de $T_{g}$ introduzindo adequados homeomorfismos marcados. Para simplificar a notação, escrevemos $\omega$ em vez de $(L, A)$ e $F^{\omega}$ em vez de $F^{L A}$, assim da definição de $F^{\omega}$ para $\omega=(L, A) \in \mathcal{R}^{6 g-6}$ temos uma única sequência de distintas $Y$-peças $Y_{i}^{\omega}, i=1, \ldots, 2 g-2$ com geodésicas da fronteira $\gamma_{i 1}^{\omega}, \gamma_{i 2}^{\omega}, \gamma_{i 3}^{\omega}$ tal que se $c_{k}=\left(c_{i \mu}, c_{j \nu}\right)$, então $l\left(\gamma_{i \mu}^{\omega}\right)=l\left(\gamma_{j \nu}^{\omega}\right)=l_{k}$.

$F^{\omega}$ é definido como o quociente

$$
F^{\omega}=Y_{1}^{\omega} \cup \ldots \cup Y_{2 g-2}^{\omega} \bmod \left(\gamma_{i \mu}^{\omega}(t)=\gamma_{j \nu}^{\omega}(\alpha-t)=: \gamma_{k}^{\omega}(t)\right), t \in \mathcal{S}^{1}, k=1 \ldots, 3 g-3
$$

Todos os $Y_{i}^{\omega}$ são assumidos ser dados na forma básica. Denotamos por $\pi^{\omega}: Y_{1}^{\omega} \cup \ldots \cup$ $Y_{2 g-2}^{\omega} \rightarrow F^{\omega}$ a projeção natural. No caso especial onde $A=(0, \ldots, 0)=A_{0}$ utilizamos $L$ em vez de $L A_{0}$ e para a superfície base (onde $L=(1, \ldots, 1)=L_{0}$ e $A=A_{0}$ ) omitimos o símbolo $\omega=(L, A)$.

Os homeomorfismos marcados serão dados pela composição $\varphi^{\omega}=\tau^{\omega} \circ \sigma^{L}$, onde $\sigma^{L}$ : $F \rightarrow F^{L}$ e $\tau^{\omega}: F^{L} \rightarrow F^{\omega}$ serão definidos a continuação.

Para $i=1, \ldots, 2 g-2$, seja $\sigma_{i}^{L}: Y_{i} \rightarrow Y_{i}^{L}$ o homeomorfismo introduzido na Definição 4.18 a qual preserva a parametrização básica das geodésicas da fronteira. Desde que todos os parâmetros de torção são zero, a seguinte aplicação é bem definida de $F$ a $F^{L}$

$$
\sigma^{L}=\left\{\pi^{L} \circ \sigma_{i}^{L} \circ \pi^{-1} \mathrm{em} \pi\left(Y_{i}\right) \quad i=1, \ldots, 2 g-2 .\right.
$$

$\sigma^{L}: F \rightarrow F^{L}$ é um homeomorfismo com a propriedade que $\sigma^{L} \circ \gamma_{k}(t)=\gamma_{k}^{L}(t), t \in$ $\mathcal{S}^{1}, k=1 \ldots, 3 g-3$.

Para definir os homeomorfismos de torção $\tau^{\omega}$, lembrar que os colares $\mathcal{C}_{k}^{\omega}=\left\{p \in F^{\omega} \mid\right.$ $\left.\rho\left(p, \gamma_{k}^{\omega}\right) \leq w_{k}^{\omega}\right\} \operatorname{com} w_{k}^{\omega}=\operatorname{arcsinh}\left\{1 / \sinh \frac{1}{2} l_{k}\right\}$ são dois a dois disjuntos e admitem coordenadas de Fermi $(\rho, t) \operatorname{com}-w_{k}^{\omega} \leq \rho \leq w_{k}^{\omega}$ e $t \in \mathcal{S}^{1}$. O sinal de $\rho$ é tal que é negativo no lado esquerdo de $\gamma_{k}$ e positivo no lado direito (com respeito à orientação de $\gamma_{k}$ ). Temos que para qualquer $A \in \omega=(L, A)$ os colares $\mathcal{C}_{k}^{L}$ e $\mathcal{C}_{k}^{\omega}$ são isométricos para cada $k=1, \ldots, 3 g-3$.

Definimos as aplicaçoes $T_{k}^{\omega}: \mathcal{C}_{k}^{L} \rightarrow \mathcal{C}_{k}^{\omega}$ (Ver Figura 4.6), utilizando as coordenadas de Fermi, como

$$
T_{k}^{\omega}(\rho, t)=\left(\rho, t+\alpha_{k} \frac{w_{k}^{\omega}+\rho}{2 w_{k}^{\omega}}\right)
$$

Se $I^{\omega}: Y_{1}^{L} \cup \ldots \cup Y_{2 g-2}^{L} \rightarrow Y_{1}^{\omega} \cup \ldots \cup Y_{2 g-2}^{\omega}$ (uniões disjuntas) é a identificação natural, 


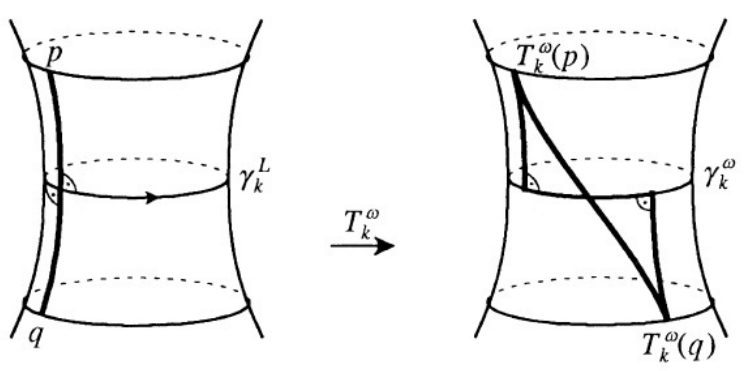

Figura 4.6:

notar que $Y_{k}^{\omega}$ e $Y_{k}^{L}$ são cópias isométricas para cada $k=1, \ldots, 3 g-3$, pois somente dependem dos comprimentos das geodésicas da fonteira as quais serão identificados de diferente maneira utilizando os parâmetros de torção $A=\left(\alpha_{1}, \ldots, \alpha_{3 g-3}\right)$, então a seguinte aplicação

$$
\tau^{\omega}= \begin{cases}T_{k}^{\omega} & \text { em } \mathcal{C}_{k}^{L}, k=1, \ldots, 3 g-3 \\ \pi^{\omega} \circ I^{\omega} \circ\left(\pi^{L}\right)^{-1} & \text { em outro lugar }\end{cases}
$$

é um homeomorfismo bem definido de $F^{L}$ a $F^{\omega}$. Notar que este homeomorfismo é o análogo aos homeomorfismo de torção (Ver (4.9) e (4.18)) para $X$-peças e no caso de assinatura $(1,1)$, respectivamente. Só que neste caso não temos um único parâmetro de torção, temos $3 g-3$, por isso é que utilizamos a identificação natural $I^{\omega}$.

Definição 4.42. Seja G um grafo. Denotamos por $S^{\omega}$ a superfície de Riemann marcada $S^{\omega}:=$ $\left(F^{\omega}, \varphi^{\omega}\right)$ onde $\varphi^{\omega}:=\tau^{\omega} \circ \sigma^{L}$. O conjunto de todas as superfícies de Riemann marcada $S^{\omega}$ baseadas no grafo $G$ e com $\omega \in \mathcal{R}^{6 g-6}$ é denotado por $T_{G}$

De 4.25) e (4.26) temos que

$$
\varphi^{\omega} \circ \gamma_{k}(t)=\gamma_{k}^{\omega}\left(t+\frac{\alpha_{k}}{2}\right), \quad t \in \mathcal{S}^{1}, \quad k=1, \ldots, 3 g-3 .
$$

Proposição 4.43. Ver [3, pág.411].

Seja S uma superfície diferenciável 2-dimensional compacta, conexa e orientávelsabado. Sejam $c_{1}, \ldots, c_{m}$ curvas fechadas, simples, homotopicamente não triviais e disjuntas duas a duas de $S$ as quais não são homotopicamente livres. Se $\gamma_{1}, \ldots, \gamma_{m}$ é outro conjunto de curvas de $S$ com as mesmas propriedades e se $c_{\mu}$ é livremente homotópico a $\gamma_{\mu}$ para $\mu=1, \ldots, m$. Então existe um homeomorfismo $\phi: S \rightarrow S$ homotópico à identidade tal que $\phi \circ c_{\mu}=\gamma_{\mu}$.

Teorema 4.44. Seja G um grafo cúbico marcado dado. Então para toda superfície de Riemann $(S, \varphi)$, existe uma única $S^{\omega}$ a qual é uma marcação equivalente $(S, \varphi)$.

Prova: Temos que determinar o valor adequado de $\omega=(L, A)$ em $S$. Pela Proposição 
4.43, podemos assumir que $\varphi\left(\gamma_{k}\right)$ é a geodésica fechada $\gamma_{k}(S), k=1, \ldots, 3 g-3$. O candidato para $L$ é

$$
L(S):=\left(l \gamma_{1}(S), \ldots, l \gamma_{3 g-3}(S)\right)
$$

Fixamos $k$ e consideremos de novo a $X$-peça $X^{k}$ como em (4.22) com a imersão isométrica $\iota_{k}: X^{k} \rightarrow F$. Esta imersão é uma isometría local, portanto podemos puxar a estrutura da superfície hiperbólica $\varphi\left(\iota_{k}\left(X^{k}\right)\right)$ sobre $X^{k}$, isto é, existe uma $X$-peça $X(S)$ (não contida em $S$ ) junto com uma imersão isométrica $\iota_{k}^{\prime}: X^{k}(S) \rightarrow S$ e um homeomorfismo $\varphi^{k}: X^{k} \rightarrow X^{k}(S)$ tal que o seguinte diagrama é commutativo.

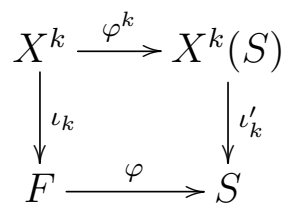

Notar que $\varphi^{k}$ mapea as $Y$-peças $Y^{i}$ e $Y^{j}$ de $X^{k}$ sobre $Y$-peças em $X^{k}(S)$. Em $X^{k}(S)$ pomos $\gamma^{k}(S)=\varphi^{k}\left(\gamma^{k}\right), \delta^{k}(S)=\varphi^{k}\left(\delta^{k}\right)$ e $\eta^{k}(S)=\varphi^{k}\left(\eta^{k}\right)$. Então

$$
\iota_{k}^{\prime}\left(\gamma^{k}(S)\right)=\gamma_{k}(S), \iota_{k}^{\prime}\left(\delta^{k}(S)\right)=\delta_{k}(S), \iota_{k}^{\prime}\left(\eta^{k}(S)\right)=\eta_{k}(S)
$$

Sejam $\gamma$ e $\gamma^{\prime} \operatorname{com} \gamma \subset \partial Y^{i}$ e $\gamma^{\prime} \subset \partial Y^{j}$ as geodésicas fronteiras de $X^{k}$ como na Figura 4.7 e as quais não são separadas por $\delta^{k}$, e seja $d$ a geodésica em $X^{k}-\delta^{k}$ de $\gamma^{\prime}$ a $\gamma$. Estas curvas são parametrizadas tal que $\delta^{k}$ é homotópico a $d \gamma d^{-1} \gamma^{\prime}$.

$\varphi^{k}(d)$ em $X^{k}(S)$ é homotópico com seus pontos extremos estando na fronteira a uma única curva $a_{2}^{\prime} b a_{2}^{-1}$ com as seguintes propiedades: $a_{2}^{\prime}$ é a geodésica de $\varphi^{k}\left(\gamma^{\prime}\right)$ a $\gamma^{k}(S) ; b$ é um arco geodésico em $\gamma^{k}(S)$ (não é simples em geral) e $a_{2}$ é a geodésica de $\varphi^{k}(\gamma)$ a $\gamma^{k}(S)$. Seja $\beta_{k}(S)$ o comprimento com sinal de $b$ (positivo se mantém a orientação de
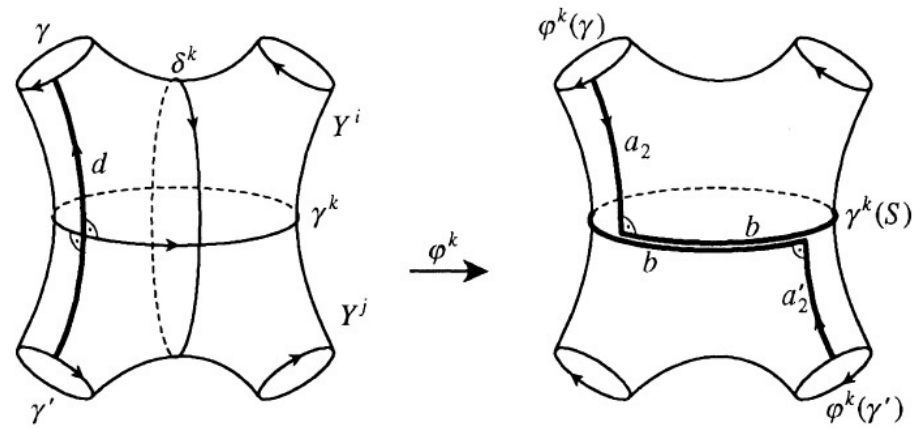

Figura 4.7: 
$\gamma^{k}(S)$ e negativo caso contrário). Definimos

$$
\alpha_{k}(S):=\beta_{k}(S) / l \gamma_{k}(S), \quad k=1, \ldots, 3 g-3
$$

assim temos o candidato para $A$

$$
A(S):=\left(\alpha_{1}(S), \ldots, \alpha_{3 g-3}(S)\right)
$$

Logo, pondo $\omega:=(L(S), A(S))$ e $\hat{S}:=S^{\omega}$. Provaremos que $S=(S, \varphi)$ e $\hat{S}$ são marcações equivalentes. De novo fixamos $k$.

Como para $S$, consideramos a imersão isométrica $\hat{\iota}_{k}: X^{k}(\hat{S}) \rightarrow \hat{S}$ com o diagrama commutativo

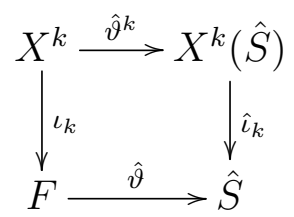

onde $\hat{\vartheta}=\varphi^{\omega}=\tau^{\omega} \circ \sigma^{L}$ com $L=L(S)$. O análogo de $a_{2}^{\prime} b a_{2}^{-1}$ é $\hat{a}_{2}^{\prime} \hat{b} \hat{a}_{2}^{-1}$. Com a definição de $\tau$ vemos que $\hat{b}$ e $b$ têm o mesmo comprimento com sinal.

Portanto temos uma isometria preservando orientação $m^{k}: X^{k}(S) \rightarrow X^{k}(\hat{S})$ a qual mapea $a_{2}^{\prime}$ a $\hat{a}_{2}^{\prime} b$ a $\hat{b}$ e $a_{2}$ a $\hat{a}_{2}$. Desde que $\delta^{k}$ é homotópico a $d \gamma d^{-1} \gamma^{\prime}$, seguemos que as geodésicas $m^{k}\left(\delta^{k}(S)\right)$ e $\delta^{k}(\hat{S})$ são homotópicos . Pela unicidade de uma geodésica em sua classe de homotopia obtemos

$$
m^{k} \circ \delta^{k}(S)=\delta^{k}(\hat{S})
$$

Observamos que a restrição $m^{k} \mid \varphi^{k}\left(Y^{i}\right)$ é a única isometria preservando orientação de $\varphi^{k}\left(Y^{i}\right)$ a $\hat{\vartheta}^{k}\left(Y^{i}\right)$ com a propiedade que $m^{k}(c)=\hat{\vartheta}^{k} \circ\left(\varphi^{k}\right)^{-1}(c)$ para cada geodésica fronteira $c$ de $\varphi^{k}\left(Y^{i}\right)$. Isto implica que as isometrias locais

$$
\hat{\iota}_{k} \circ m^{k} \circ\left(\iota_{k}^{\prime}\right)^{-1}: \iota_{k}^{\prime}\left(\operatorname{int} X^{k}(S)\right) \rightarrow \hat{\iota}_{k}\left(\operatorname{int} X^{k}(\hat{S})\right) \quad k=1, \ldots, 3 g-3
$$

definem uma única isometria $m: S \rightarrow \hat{S}$ satisfazendo

$$
m \circ \delta_{k}(S)=\delta_{k}(\hat{S}) \quad k=1, \ldots, 3 g-3 .
$$

Pelo Teorema $4.40, S$ e $\hat{S}=S^{\omega}$ são marcações equivalentes.

Finalmente, se $S^{\omega}, S^{\omega^{\prime}} \in T_{G}$ são marcações equivalentes, então temos uma isometria $m: S^{\omega} \rightarrow S^{\omega^{\prime}}$ tal que $m \circ \varphi^{\omega}$ é isotópico a $\varphi^{\omega^{\prime}}$. Consequentemente, $m$ mapea $\Omega_{G}\left(S^{\omega}\right)$ 
sobre $\Omega_{G}\left(S^{\omega^{\prime}}\right)$ tal que $l \Omega_{G}\left(S^{\omega}\right)=l \Omega_{G}\left(S^{\omega^{\prime}}\right)$. Esta equação implica que $L=L^{\prime}$ e pela Proposição 4.27 temos que $A=A^{\prime}$.

Corolário 4.45. Duas superfícies de Riemann marcadas $S$ e $S^{\prime}$ são equivalentes marcadas se e somente se $l \Omega_{G}(S)=l \Omega_{G}\left(S^{\prime}\right)$.

Fixado $g$. O Teorema 4.44 diz que para todo grafo cúbico marcado $G$ com $2 g-2$ vértices, o conjunto

$$
T_{G}=\left\{S^{\omega}=S_{G}^{\omega} \mid \omega \in \mathcal{R}^{6 g-6}\right\}
$$

é um modelo para $T_{g}$.

Para a simplicidade da notação, denotamos também por $S$ a classe de equivalência marcada de uma superfície de Riemann marcada $S=(S, \varphi)$. Assim, se $S \in T_{g}$, identificamos $S$ com qualquer de seus representantes e tratamos com $S$ como uma superfície de Riemann.

Definição 4.46. Se G um grafo como acima. Para todo $S \in T_{g}$ seja $\omega(S)=\omega_{G}(S)$ o único $\omega \in$ $\mathcal{R}^{6 g-6}$ tal que $S$ é equivalentemente marcado a $S_{G}^{\omega}$. As componentes de $\omega_{G}(S)$ são chamadaos as coordenadas de Fenchel-Nielsen de S.

A topologia de $T_{g}$ é definido por meio das funções coordenadas $\omega_{G}: T_{g} \rightarrow \mathcal{R}^{6 g-6}$

Lema 4.47. Seja $G$ dado e seja $\mathcal{L}=\left\{l \Omega_{G}(S) \mid S \in T_{g}\right\}$. Existe uma vizinhança aberta $\mathcal{D}$ de $\mathcal{L}$ em $\mathbb{R}^{9 g-9}$ junto com uma aplicação analítica real $\mathcal{A}=\mathcal{A}_{G}: \mathcal{D} \rightarrow \mathbb{R}^{6 g-6}$ tal que $\omega_{G}(S)=\mathcal{A}_{G}\left(l \Omega_{G}(S)\right)$ para todo $S \in T_{g}$.

Prova: Provamos isto no modelo $T_{G}$. As primeiras $3 g-3$ coordenadas, formando um vetor $L=\left(l_{1}, \ldots, l_{3 g-3}\right)$ são parte de $l \Omega\left(S^{\omega}\right)$. Basta provar que cada parâmetro de torção $\alpha_{k}$ é uma função analítica real de $l \Omega_{G}\left(S^{\omega}\right)$. Para isto consideramos, como no Teorema 4.44 a imersão isométrica $\iota_{k}^{\prime}: X^{k}\left(S^{\omega}\right) \rightarrow S^{\omega}$ e os levantamentos $\gamma^{k}\left(S^{\omega}\right), \delta^{k}\left(S^{\omega}\right)$ e $\eta^{k}\left(S^{\omega}\right)$ de $\gamma_{k}\left(S^{\omega}\right), \delta_{k}\left(S^{\omega}\right)$ e $\eta_{k}\left(S^{\omega}\right)$ junto com as geodésicas da fronteira de $X^{k}\left(S^{\omega}\right)$ as quais formam o sistema de curvas como em (4.12). As presentes curvas $\gamma^{k}\left(S^{\omega}\right), \delta^{k}\left(S^{\omega}\right)$ e $\eta^{k}\left(S^{\omega}\right)$ são $\gamma^{\alpha}, \delta^{\alpha}$ e $\eta^{\alpha}$ de (4.12), respectivamente, $\operatorname{com} \alpha=\alpha_{k}$, e as geodésicas da fronteira de $X^{k}\left(S^{\omega}\right)$ são $\gamma_{2}, \gamma_{3}, \gamma_{2}^{\prime}, \gamma_{3}^{\prime}$. Agora $\alpha_{k}$ é determinado pela função analítica $\mathscr{A}$ da Proposição 4.28 .

Teorema 4.48. Para qualquer curva fechada $\beta$ na superfície base $F$, a função $\omega \mapsto l \beta\left(S^{\omega}\right), \omega \in$ $\mathcal{R}^{6 g-6}$ é analítica real.

Prova: Fixamos $\omega_{0}$. Calculamos $l \beta\left(S^{\omega}\right)$ para $\omega$ em uma vizinhança de $\omega_{0}$. Isto será realizado em 3 passos. Primeiro substituímos $\beta\left(S^{\omega}\right)$ por um polígono geodésico reto 
o qual consideramos como um laço geodésico por partes em algum ponto base $p\left(S^{\omega}\right)$. Logo utilizamos este polígono para calcular o laço geodésico $\beta^{*}\left(S^{\omega}\right)$ na sua classe de homotopia com ponto base fixado e finalmente calculamos $\beta\left(S^{\omega}\right)$ em termos de $\beta^{*}\left(S^{\omega}\right)$. Escolhemos como ponto base

$$
p\left(S^{\omega}\right)=\gamma_{1}^{\omega}\left(\frac{1}{2} \alpha_{1}+\frac{1}{4}\right),
$$

onde $\alpha_{1}$ é o parâmetro de torção em $\gamma_{1}^{\omega}=\gamma_{1}\left(S^{\omega}\right)$. Agora escolhemos um laço geodésico $\beta^{*}\left(S^{\omega_{0}}\right)$ com ponto base $p\left(S^{\omega_{0}}\right)$ a qual é livremente homotópico a $\beta\left(S^{\omega_{0}}\right)$ e tal que forma um ângulo $\theta<\pi \operatorname{em~} p\left(S^{\omega_{0}}\right)$.

O laço geodésico por partes com ângulos retos é definido como segue. Consideremos os hexágonos retos de $Y_{1}, \ldots Y_{2 g-2}$ em $S^{\omega_{0}}$ (ladrilhamento de $S^{\omega_{0}}$ ). Seja

$$
\bar{\beta}^{\omega_{0}}=b_{1}^{\omega_{0}} \cdot \ldots \cdot b_{2 n+1}^{\omega_{0}}
$$

um laço geodésico por partes com ponto base $p\left(S^{\omega_{0}}\right)$ que corre ao longo dos lados dos hexágonos acima tal que o seguinte se cumpre:

(2) $\bar{\beta}^{\omega}$ é homotópico, com ponto base fixado, a $\beta^{*}\left(S^{\omega_{0}}\right)$.

(3) cada lado $b_{\nu}^{\omega_{0}}$, com $\nu$ impar, corre ao longo algum $\gamma_{k}^{\omega_{0}}$ ( $k$ dependendo em $\nu$ ).

(4) cada lado $b_{\nu}^{\omega_{0}}$, com $\nu$ par, é um das três perpendiculares comuns entre as geodésicas das fonteiras de algum $Y_{i}$.

Em (3) $b_{\nu}^{\omega_{0}}$ é permitido ter comprimento zero (se o parâmetro de torção em $\gamma_{k}^{\omega_{0}}$ é um múltiplo inteiro de $\frac{1}{2}$ ). Também se permite $b_{\nu}^{\omega}$ correr varias vezes ao redor de $\gamma_{k}^{\omega_{0}}$. O ângulo de $\bar{\beta}^{\omega_{0}}$ em $p\left(S^{\omega_{0}}\right)$ é ou 0 ou $\pi$. Todos os outros ângulos de $\bar{\beta}^{\omega_{0}}$ são retos. Os lados com um número para têm comprimento positivo. Para os demais lados, isto é, para aquilos em $\gamma_{1}^{\omega_{0}}, \ldots, \gamma_{3 g-3}^{\omega_{0}}$ introduzimos comprimentos com sinal: se $b_{\nu}^{\omega_{0}} \mathrm{em} \gamma_{k}^{\omega_{0}}$ tem a mesma orientação como $\gamma_{k}^{\omega_{0}}$ (com respeito à orientação do laço $\bar{\beta}^{\omega_{0}}$ ) então $l\left(b_{\nu}^{\omega_{0}}\right) \geq 0$, caso contrário $l\left(b_{\nu}^{\omega_{0}}\right) \leq 0$.

Agora consideremos $\omega$ perto de $\omega_{0}$ e consideremos o correspondente ladrilhamento de $S^{\omega}$. Ele difere apenas um pouco do ladrilhamento de $S^{\omega_{0}}$ e temos o correspondente laço geodésico por partes com ângulos retos

$$
\overline{\beta^{\omega}}=b_{1}^{\omega} \cdot \ldots \cdot b_{2 n+1}^{\omega}
$$

com ponto base $p\left(S^{\omega}\right)$ o qual difere apenas um pouco de $\bar{\beta}^{\omega_{0}}$. Pela convenção do sinal, os comprimentos de $b_{1}^{\omega}, \ldots, b_{2 n+1}^{\omega}$ são funções contínuas e portanto analíticas reais de $\omega$.

Os homeomorfismos marcados $\varphi^{\omega}: F \rightarrow S^{\omega}$ no modelo $T_{G}$ são definidos de tal maneira que 
(5)

$$
\varphi^{\omega} \circ\left(\varphi^{\omega_{0}}\right)^{-1}\left(p\left(S^{\omega_{0}}\right)\right)=p\left(S^{\omega}\right)
$$

e com a definição de $\varphi^{\omega}$ vemos que

(6) $\bar{\beta}^{\omega}$ é homotópico, com ponto base fixado, a $\varphi^{\omega} \circ\left(\varphi^{\omega}\right)^{-1}\left(\bar{\beta}^{\omega_{0}}\right)$.

Daqui, a geodésica fechada $\beta=\beta\left(S^{\omega}\right)$ esta na classe de homotopia livre de $\bar{\beta}=\bar{\beta}^{\omega}$. Portanto é suficiente encontrar uma função analítica que da o comprimento de $\beta$ em termos de $b_{1}=b_{1}^{\omega}, \ldots, b_{2 n+1}=b_{2 n+1}^{\omega}$. Para facilitar a notação fazemos isto sem escrever o simbolo $\omega$.

Sem mudar a notação levantamos $\bar{\beta}$ no disco unitário $\Delta$ de tal maneira que $b_{1}$ esta no eixo real positivo com ponto inicial $p_{0}$ na origem. Para $\nu=1, \ldots, 2 n+1$ denotamos por $p_{\nu}$ o ponto final de $b_{\nu}$ e sejam $B_{\nu}^{-}$e $B_{\nu}^{+}$os pontos extremos em $\partial \Delta$ (pontos no infinito) da extensão geodésica do arco $b_{\nu}$. Se em $S^{\omega}, b_{\nu}$ esta em $\gamma_{k}^{\omega}$, então em $\Delta, b_{\nu}$ esta em um levantamento de $\gamma_{k}^{\omega}$, e $B_{\nu}^{-}$e $B_{\nu}^{+}$são os pontos extremos deste levantamento.

Pode-se ver que os pontos $p_{\nu}, B_{\nu}^{-}$e $B_{\nu}^{+}$são calculáveis em termos de $l\left(b_{1}\right), \ldots, l\left(b_{2 n+1}\right)$ por meio de funções analíticas (por indução sobre $\nu$ ).

Seja $\beta^{*}$ o arco geodésico em $\Delta$ de $p_{0}$ a $p_{2 n+1}$. Então $l\left(\beta^{*}\right)$ e os dois ângulos entre $\beta^{*}$ e $b_{1}$, e $\beta^{*}$ e $b_{2 n+1}$ são calculáveis por funções analíticas de $l\left(b_{1}\right), \ldots, l\left(b_{2 n+1}\right)$.

Em $S^{\omega}$ a projeção $\beta^{*}(S)$ de $\beta^{*}$ é um laço geodésico em $p\left(S^{\omega}\right)$ a qual é homotópico com ponto base fixado a $\bar{\beta}=\bar{\beta}^{\omega}$. Junto com (6) obtemos:

(7) $\beta^{*}\left(S^{\omega}\right)$ é homotópico com ponto base fixado a $\varphi^{\omega} \circ\left(\varphi^{\omega_{0}}\right)^{-1}\left(\beta^{*}\left(S^{\omega_{0}}\right)\right)$.

E das obsevações acima

(8) O comprimento de $\beta^{*}\left(S^{\omega}\right)$ e os ângulos no ponto base entre $\beta^{*}\left(S^{\omega}\right)$ e $\gamma_{1}^{\omega}$ são funções analíticas de $\omega$.

Mais uma vez levantamos (estendido periodicamente) as curvas fechadas livremente

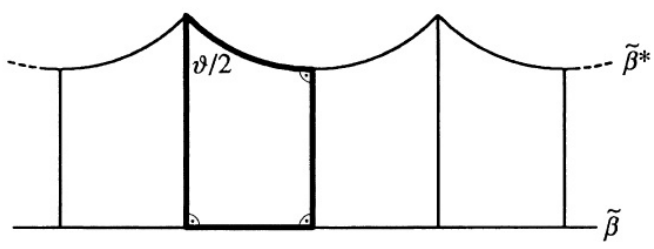

Figura 4.8:

homotópicas $\beta^{*}$ e $\beta$ no recobrimento universal $\Delta$ para obter curvas homotópicas $\tilde{\beta}^{*} \mathrm{e}$ $\tilde{\beta}$ com os mesmos pontos extremos no infinito. Para $\omega$ suficientemente perto de $\omega_{0} \mathrm{o}$ ângulo $\theta$ do laço $\beta^{*}$ no ponto base é positivo. Portanto $\tilde{\beta}^{*}$ e $\tilde{\beta}$ são disjuntos e limitam uma faixa a qual é pavimentada por triretângulos isométricos (Ver Figura 4.8). O ângulo agudo do triretângulo é $\theta / 2$ e os lados são $x, \frac{1}{2} l\left(\beta^{*}\right), y, \frac{1}{2} l(\beta)$. Da Proposição 1.50 (ii) obtemos $\cosh \frac{1}{2} l(\beta)=\cosh \frac{1}{2} l\left(\beta^{*}\right) \sin \frac{1}{2} \theta$ e junto com (8) se prova o teorema.

Teorema 4.49. Se dois grafos $G$ e $G^{\prime}$ são dados com funções coordenadas $\omega_{G}$ e $\omega_{G^{\prime}}$, respectiva- 
mente, então $\omega_{G} \circ \omega_{G^{\prime}}: \mathcal{R}^{6 g-6} \rightarrow \mathcal{R}^{6 g-6}$ é um difeomorfismo analítico real.

Prova: Temos provado que as coordenadas de Fenchel-Nielsen são funções analiticas de $l \Omega_{G}(S)$ (Lema 4.47) e que os comprimentos geodésicos são funções analiticas das coordenadas de Fenchel-Nielsen (Teorema 4.48).

Concluímos introduzindo em $T_{g}$ a única estrutura analítica real tal que as aplicações $\omega_{G}: T_{g} \rightarrow \mathcal{R}^{6 g-6}$ são difeomorfismos analíticos reais. 


\section{Referências Bibliográficas}

[1] A. Beardon, The Geometry of Discrete Groups, Springer-Verlag (1983).

[2] K. Burns, M. Gidea, Differential geometry and topology with a view to dynamical systems, Chapman-Hall (2005).

[3] P. Buser, Geometry and spectra of compact Riemann surface, Birkäuser Boston (1992).

[4] B. Farb, D. Margalit, A primer on mapping class groups Version 5.0, (2011). http:/ / www.math.uchicago.edu/ margalit/mcg/mcgv50.pdf

[5] O. Forster, Lectures on Riemann Surfaces, Springer-Verlag (1999).

[6] M. Hirsch, Differential Topology, Springer-Verlag, Berlin and New York (1976).

[7] Y. Imayoshi, M. Taniguchi, An introduction to Teichmüller spaces, Springer-Verlag (1992).

[8] G. Jones, D. Singerman, Complex Functions, Cambridge University Press (1987).

[9] J. Jost, Compact Riemann Surfaces, Springer-Verlag, Berlin (2006).

[10] S. Katok, Fuchsian Groups, Chicago Lecture in Mathematics (1992).

[11] W. Massey, A basic course in algebraic topology, Springer-Verlag (1991).

[12] Y. Matsushima, Differentiable Manifolds, Pure and Applied Mathematics (1972). 